\title{
DERIVED DEFORMATIONS OF ARTIN STACKS
}

\author{
J.P.PRIDHAM
}

\begin{abstract}
We generalise the techniques of [Pri5] to describe derived deformations in simplicial categories. This allows us to consider deformation problems with higher automorphisms, such as chain complexes (which have homotopies) and stacks (which have 2-automorphisms). We also give a general approach for studying deformations of diagrams.
\end{abstract}

\section{INTRODUCTION}

This paper is motivated by the wish to describe derived deformations of an algebraic stack. In [Ols2] and [Aok], it was shown that deforming an algebraic stack can be regarded as a special case of deforming a simplicial algebraic space. The category of simplicial spaces has a natural simplicial structure (meaning that the Hom-sets can be enriched to give simplicial sets), and the 2-groupoid of deformations of an algebraic stack can be recovered from this simplicial structure.

After reviewing background material from [Pri4] in $\S 1$, we introduce derived deformation complexes (DDCs) In Section 2; these extend the SDCs of [Pri2] to simplicial categories. We then adapt the various constructions of [Pri4], showing how to associate derived deformation functors to DDCs, and how to compare them with derived deformation functors coming from SDCs.

Section 3 adapts the ideas of [Pri2], showing how to associate DDCs to bialgebraic deformation problems in simplicial categories. In $\S 3.3$, we show how deformations of morphisms and diagrams can be used to compare deformations of weakly equivalent objects.

Several simple examples of such problems are considered in Section 4: chain complexes (with more interesting variants in Remarks 4.7), simplicial complexes and simplicial algebras.

The motivating example of algebraic stacks is finally considered in Section 5. We first describe derived deformations of simplicial affine schemes ( $\S 5.1)$, then show in $\S 5.2$ how to adapt this to describe derived deformations of an algebraic stack $\mathfrak{X}$, with an indication in Remark 5.28 of how this approach also works for Artin $n$-stacks. The idea is to consider derived deformations of a suitable hypercovering $X \bullet$ of $\mathfrak{X}$. To see that this does, indeed, extend the 2-groupoid of deformations of $\mathfrak{X}$, we establish comparisons with Olsson's Extgroups of the cotangent complex $(\S 5.2 .1)$ and Aoki's description of the deformation 2groupoid $(\S 5.2 .2)$.

\section{Contents}

Introduction

1. Derived deformation functors 2

1.1. Simplicial Artinian rings 2

1.2. Properties of morphisms 3

1.3. Derived deformation functors 3

1.4. Quotient spaces 4

1.5. Cohomology and obstructions 5

The author was supported during this research by Trinity College, Cambridge. 
1.6. Model structures 6

2. Derived deformation complexes $\quad 7$

2.1. Definitions $r$

2.2. Comparison with SDCs 10

3. Constructing DDCs 12

3.1. Simplicial monadic adjunctions 12

3.2. The construction 13

3.3. Deformations of diagrams and invariance under weak equivalence 16

4. Examples 18

4.1. Chain complexes 18

4.2. Simplicial complexes 20

4.3. Simplicial algebras 21

5. Deformations of Artin stacks and simplicial schemes 24

5.1. Cosimplicial algebras 24

5.2. Quasi-compact, quasi-separated stacks 30

5.3. Arbitrary algebraic stacks 34

Bibliography $\quad 36$

$\begin{array}{ll}\text { References } & 36\end{array}$

\section{Derived DeFormation FunCtors}

With the exception of $\S 1.4$, the definitions and results in this section can all be found in [Pri4]. Fix a complete local Noetherian ring $\Lambda$, with maximal ideal $\mu$ and residue field $k$.

\subsection{Simplicial Artinian rings.}

Definition 1.1. Let $\mathcal{C}_{\Lambda}$ denote the category of local Artinian $\Lambda$-algebras with residue field $k$. We define $s \mathcal{C}_{\Lambda}$ to be the category of Artinian simplicial local $\Lambda$-algebras, with residue field $k$.

Definition 1.2. Given a simplicial complex $V_{\bullet}$, recall that the normalised chain complex $N^{s}(V)$ • is given by $N^{s}(V)_{n}:=\bigcap_{i>0} \operatorname{ker}\left(\partial_{i}: V_{n} \rightarrow V_{n-1}\right)$, with differential $\partial_{0}$. The simplicial Dold-Kan correspondence says that $N^{s}$ gives an equivalence of categories between simplicial complexes and non-negatively graded chain complexes in any abelian category. Where no ambiguity results, we will denote $N^{s}$ by $N$.

Lemma 1.3. A simplicial complex $A$. of local $\Lambda$-algebras with residue field $k$ and maximal ideal $\mathfrak{m}(A)$. is Artinian if and only if:

(1) the normalisation $N(\cot A)$ of the cotangent space $\cot A:=\mathfrak{m}(A) /\left(\mathfrak{m}(A)^{2}+\mu \mathfrak{m}(A)\right)$ is finite-dimensional (i.e. concentrated in finitely many degrees, and finitedimensional in each degree).

(2) For some $n>0, \mathfrak{m}(A)^{n}=0$.

Proof. [Pri4] Lemma 1.16

As in [Gro], we say that a functor is left exact if it preserves all finite limits. This is equivalent to saying that it preserves final objects and fibre products.

Definition 1.4. Define $\mathrm{Sp}$ to be the category of left-exact functors from $\mathcal{C}_{\Lambda}$ to Set. Define $c \mathrm{Sp}$ to be the category of left-exact functors from $s \mathcal{C}_{\Lambda}$ to Set.

Definition 1.5. Given a functor $F: \mathcal{C}_{\Lambda} \rightarrow$ Set, we write $F: s \mathcal{C}_{\Lambda} \rightarrow$ Set to mean $A \mapsto F\left(A_{0}\right)$ (corresponding to the inclusion $\mathrm{Sp} \hookrightarrow c \mathrm{Sp}$ ). 


\subsection{Properties of morphisms.}

Definition 1.6. As in [Man], we say that a functor $F: \mathcal{C}_{\Lambda} \rightarrow$ Set is smooth if for all surjections $A \rightarrow B$ in $\mathcal{C}_{\Lambda}$, the map $F(A) \rightarrow F(B)$ is surjective.

Definition 1.7. We say that a map $f: A \rightarrow B$ in $s \hat{\mathcal{C}}_{\Lambda}$ is acyclic if $\pi_{i}(f): \pi_{i}(A) \rightarrow \pi_{i}(B)$ is an isomorphism of pro-Artinian $\Lambda$-modules for all $i . f$ is said to be surjective if each $f_{n}: A_{n} \rightarrow B_{n}$ is surjective.

Note that for any simplicial abelian group $A$, the homotopy groups can be calculated by $\pi_{i} A \cong \mathrm{H}_{i}(N A)$, the homology groups of the normalised chain complex. These in turn are isomorphic to the homology groups of the unnormalised chain complex associated to $A$.

Definition 1.8. We define a small extension $e: I \rightarrow A \rightarrow B$ in $s \mathcal{C}_{\Lambda}$ to consist of a surjection $A \rightarrow B$ in $s \mathcal{C}_{\Lambda}$ with kernel $I$, such that $\mathfrak{m}(A) \cdot I=0$. Note that this implies that $I$ is a simplicial complex of $k$-vector spaces.

Lemma 1.9. Every surjection in $s \mathcal{C}_{\Lambda}$ can be factorised as a composition of small extensions. Every acyclic surjection in $s_{\mathcal{C}_{\Lambda}}$ can be factorised as a composition of acyclic small extensions.

Proof. [Pri4] Lemma 1.23.

Definition 1.10. We say that a morphism $\alpha: F \rightarrow G$ in $c$ Sp is smooth if for all small extensions $A \rightarrow B$ in $s \mathcal{C}_{\Lambda}$, the map $F(A) \rightarrow F(B) \times_{G(B)} G(A)$ is surjective.

Similarly, we call $\alpha$ quasi-smooth if for all acyclic small extensions $A \rightarrow B$ in $s \mathcal{C}_{\Lambda}$, the map $F(A) \rightarrow F(B) \times_{G(B)} G(A)$ is surjective.

Lemma 1.11. A morphism $\alpha: F \rightarrow G$ in $\mathrm{Sp}$ is smooth if and only if the induced morphism between the objects $F, G \in c \mathrm{Sp}$ is quasi-smooth, if and only if it is smooth.

Proof. [Pri4] Lemma 1.31.

\subsection{Derived deformation functors.}

Definition 1.12. Define the $s c \mathrm{Sp}$ to be the category of left-exact functors from $s \mathcal{C}_{\Lambda}$ to the category $\mathbb{S}$ of simplicial sets. This is equivalent to the category of simplicial cosimplicial objects in Sp.

Define $s$ Sp to be the category of left-exact functors from $\mathcal{C}_{\Lambda}$ to $\mathbb{S}$.

Definition 1.13. A morphism $\alpha: F \rightarrow G$ in $s c$ Sp is said to be smooth if

(S1) for every acyclic surjection $A \rightarrow B$ in $s \mathcal{C}_{\Lambda}$, the map $F(A) \rightarrow F(B) \times_{G(B)} G(A)$ is a trivial fibration in $\mathbb{S}$;

(S2) for every surjection $A \rightarrow B$ in $s \mathcal{C}_{\Lambda}$, the map $F(A) \rightarrow F(B) \times_{G(B)} G(A)$ is a surjective fibration in $\mathbb{S}$.

A morphism $\alpha: F \rightarrow G$ in $s c$ Sp is said to be quasi-smooth if it satisfies (S1) and

(Q2) for every surjection $A \rightarrow B$ in $s \mathcal{C}_{\Lambda}$, the map $F(A) \rightarrow F(B) \times_{G(B)} G(A)$ is a fibration in $\mathbb{S}$.

Definition 1.14. Given $A \in s \mathcal{C}_{\Lambda}$ and a finite simplicial set $K$, define $A^{K} \in \mathcal{C}_{\Lambda}$ by

$$
\left(A^{K}\right)_{i}:=\operatorname{Hom}_{\mathbb{S}}\left(K \times \Delta^{i}, A\right) \times_{\mathrm{Hom}_{\mathrm{Set}}\left(\pi_{0} K, k\right)} k .
$$

Definition 1.15. Given $F \in s c \mathrm{Sp}$, define $\underline{F}: s \mathcal{C}_{\Lambda} \rightarrow \mathbb{S}$ by

$$
\underline{F}(A)_{n}:=F_{n}\left(A^{\Delta^{n}}\right) \text {. }
$$

For $F \in c \mathrm{Sp}$, we may regard $F$ as an object of $s c \mathrm{Sp}$ (with the constant simplicial structure), and then define $\underline{F}$ as above. 
Lemma 1.16. A map $\alpha: F \rightarrow G$ in cSp is smooth (resp. quasi-smooth) if and only if the induced map of functors $\underline{\alpha}: \underline{F} \rightarrow \underline{G}$ is smooth (resp. quasi-smooth) in scSp.

Proof. [Pri4] Lemma 1.36.

The following Lemma will provide many examples of functors which are quasi-smooth but not smooth.

Lemma 1.17. If $F \rightarrow G$ is a quasi-smooth map of functors $F, G: s \mathcal{C}_{\Lambda} \rightarrow \mathbb{S}$, and $K \rightarrow L$ is a cofibration in $\mathbb{S}$, then

$$
F^{L} \rightarrow F^{K} \times_{G^{K}} G^{L}
$$

is quasi-smooth.

Proof. This is an immediate consequence of the fact that $\mathbb{S}$ is a simplicial model category, following from axiom SM7, as given in [GJ] §II.3.

The following lemma is a consequence of standard properties of fibrations and trivial fibrations in $\mathbb{S}$.

Lemma 1.18. If $F \rightarrow G$ is a quasi-smooth map of functors $F, G: s \mathcal{C}_{\Lambda} \rightarrow \mathbb{S}$, and $H \rightarrow G$ is any map of functors, then $F \times{ }_{G} H \rightarrow H$ is quasi-smooth.

Definition 1.19. A map $\alpha: F \rightarrow G$ of functors $F, G: \mathcal{C}_{\Lambda} \rightarrow \mathbb{S}$ is said to be smooth (resp. quasi-smooth, resp. trivially smooth) if for all surjections $A \rightarrow B$ in $\mathcal{C}_{\Lambda}$, the maps

$$
F(A) \rightarrow F(B) \times_{G(B)} G(A)
$$

are surjective fibrations (resp. fibrations, resp. trivial fibrations).

Proposition 1.20. A map $\alpha: F \rightarrow G$ of left-exact functors $F, G: \mathcal{C}_{\Lambda} \rightarrow \mathbb{S}$ is smooth if and only if the maps $F_{n} \stackrel{\alpha_{n}}{\longrightarrow} G_{n}$ of functors $F_{n}, G_{n}: \mathcal{C}_{\Lambda} \rightarrow$ Set are all smooth.

Proof. [Pri4] Proposition 1.39.

Proposition 1.21. If a morphism $F \stackrel{\alpha}{\rightarrow} G$ of left-exact functors $F, G: s \mathcal{C}_{\Lambda} \rightarrow \mathbb{S}$ is such that the maps

$$
\theta: F(A) \rightarrow F(B) \times_{G(B)} G(A)
$$

are surjective fibrations for all acyclic small extensions $A \rightarrow B$, then $\underline{\alpha}: \underline{F} \rightarrow \underline{G}$ is quasismooth (resp. smooth) if and only if $\theta$ is a fibration (resp. surjective fibration) for all small extensions $A \rightarrow B$.

Proof. [Pri4] Proposition 1.63.

Definition 1.22. We will say that a morphism $\alpha: F \rightarrow G$ of quasi-smooth objects of $s c$ Sp is a weak equivalence if, for all $A \in s \mathcal{C}_{\Lambda}$, the maps $\pi_{i} F(A) \rightarrow \pi_{i} G(A)$ are isomorphisms for all $i$.

\subsection{Quotient spaces.}

Definition 1.23. Given functors $X: s \mathcal{C}_{\Lambda} \rightarrow \mathbb{S}$ and $G: s \mathcal{C}_{\Lambda} \rightarrow s \mathrm{Gp}$, together with a right action of $G$ on $X$, define the quotient space by

$$
[X / G]_{n}=\left(X \times{ }^{G} W G\right)_{n}=X_{n} \times G_{n-1} \times G_{n-2} \times \ldots G_{0},
$$

with operations as standard for universal bundles (see [GJ] Ch. V). Explicitly:

$\partial_{i}\left(x, g_{n-1}, g_{n-2}, \ldots, g_{0}\right)=\left\{\begin{array}{cc}\left(\partial_{0} x * g_{n-1}, g_{n-2}, \ldots, g_{0}\right) & i=0 ; \\ \left(\partial_{i} x, \partial_{i-1} g_{n-1}, \ldots,\left(\partial_{0} g_{n-i}\right) g_{n-i-1}, g_{n-i-2}, \ldots, g_{0}\right) & 0<i<n ; \\ \left(\partial_{n} x, \partial_{n-1} g_{n-1}, \ldots, \partial_{1} g_{1}\right) & i=n ;\end{array}\right.$

$\sigma_{i}\left(x, g_{n-1}, g_{n-2}, \ldots, g_{0}\right)=\left(\sigma_{i} x, \sigma_{i-1} g_{n-1}, \ldots, \sigma_{0} g_{n-i}, e, g_{n-i-1}, g_{n-i-2}, \ldots, g_{0}\right)$.

The space $[\bullet / G]$ is also denoted $\bar{W} G$, and is a model for the classifying space $B G$ of $G$. 
Lemma 1.24. If $G: s \mathcal{C}_{\Lambda} \rightarrow s \mathrm{Gp}$ is smooth, then $\bar{W} G$ is smooth.

Proof. For any surjection $A \rightarrow B$, we have $G(A) \rightarrow G(B)$ fibrant and surjective on $\pi_{0}$, which by [GJ] Corollary V.6.9 implies that $\bar{W} G(A) \rightarrow \bar{W} G(B)$ is a fibration. If $A \rightarrow B$ is also acyclic, then everything is trivial by properties of $\bar{W}$ and $G$.

Remark 1.25. Observe that this is our first example of a quasi-smooth functor which is not a right Quillen functor for the simplicial model structure. The definitions of smoothness and quasi-smoothness were designed with $\bar{W} G$ in mind.

Lemma 1.26. If $X$ is quasi-smooth, then so is $[X / G] \rightarrow \bar{W} G$.

Proof. This follows from the observation that for any fibration (resp. trivial fibration) $Z \rightarrow Y$ of $G$-spaces, $[Z / G] \rightarrow[Y / G]$ is a fibration (resp. trivial fibration).

Corollary 1.27. If $X$ is quasi-smooth and $G$ smooth, then $[X / G]$ is quasi-smooth.

Proof. Consider the fibration $X \rightarrow[X / G] \rightarrow \bar{W} G$.

1.5. Cohomology and obstructions. Given a quasi-smooth morphism $\alpha: F \rightarrow G$ in $s c \mathrm{Sp}$, there exist $k$-vector spaces $\mathrm{H}^{i}(F / G)$ for all $i \in \mathbb{Z}$.

By [Pri4] Corollary 1.46, these have the property that for any simplicial $k$-vector space $V$ with finite-dimensional normalisation,

$$
\pi_{m}\left(F(k \oplus V) \times_{G(k \oplus V)}\{0\}\right) \cong \mathrm{H}^{-m}(F / G \otimes V),
$$

where $V^{2}=0$ and

$$
H^{i}(F / G \otimes V):=\bigoplus_{n \geq 0} \mathrm{H}^{i+n}(F / G) \otimes \pi_{n}(V) .
$$

If $G=\bullet$ (the one-point set), we write $\mathrm{H}^{j}(F):=\mathrm{H}^{j}(F / \bullet)$.

We now have the following characterisation of obstruction theory:

Theorem 1.28. If $\alpha: F \rightarrow G$ in $s c \mathrm{Sp}$ is quasi-smooth, then for any small extension $e: I \rightarrow A \stackrel{f}{\rightarrow} B$ in $s \mathcal{C}_{\Lambda}$, there is a sequence of sets

$$
\pi_{0}(F A) \stackrel{f_{*}}{\longrightarrow} \pi_{0}\left(F B \times_{G B} G A\right) \stackrel{o_{e}}{\longrightarrow} \mathrm{H}^{1}(F / G \otimes I)
$$

exact in the sense that the fibre of $o_{e}$ over 0 is the image of $f_{*}$. Moreover, there is a group action of $\mathrm{H}^{0}(F / G \otimes I)$ on $\pi_{0}(F A)$ whose orbits are precisely the fibres of $f_{*}$.

For any $y \in F_{0} A$, with $x=f_{*} y$, the fibre of $F A \rightarrow F B \times_{G B} G A$ over $x$ is isomorphic to $\operatorname{ker}(\alpha: F I \rightarrow G I)$, and the sequence above extends to a long exact sequence

$$
\begin{aligned}
& \cdots \stackrel{f_{*}}{\longrightarrow} \pi_{n}\left(F B \times_{G B} G A, x\right) \stackrel{o_{e}}{\longrightarrow} \mathrm{H}^{1-n}(F / G \otimes I) \stackrel{\partial_{e}}{\longrightarrow} \pi_{n-1}(F A, y) \stackrel{f_{*}}{\longrightarrow} \cdots \\
& \cdots \stackrel{f_{*}}{\longrightarrow} \pi_{1}\left(F B \times_{G B} G A, x\right) \stackrel{o_{e}}{\longrightarrow} \mathrm{H}^{0}(F / G \otimes I) \stackrel{-* y}{\longrightarrow} \pi_{0}(F A) .
\end{aligned}
$$

Proof. [Pri4] Theorem 1.45.

Corollary 1.29. A map $\alpha: F \rightarrow G$ of quasi-smooth $F, G \in$ scSp is a weak equivalence if and only if the maps $\mathrm{H}^{j}(\alpha): \mathrm{H}^{j}(F) \rightarrow \mathrm{H}^{j}(G)$ are all isomorphisms.

Corollary 1.30. If $\alpha: F \rightarrow G$ is quasi-smooth in scSp, then $\alpha$ is smooth if and only if $\mathrm{H}^{i}(F / G)=0$ for all $i>0$.

Proposition 1.31. Let $X, Y, Z: s \mathcal{C}_{\Lambda} \rightarrow \mathbb{S}$ be left-exact functors, with $X \stackrel{\alpha}{\rightarrow} Y$ and $Y \stackrel{\beta}{\rightarrow} Z$ quasi-smooth. There is then a long exact sequence

$$
\ldots \stackrel{\partial}{\rightarrow} \mathrm{H}^{j}(X / Y) \rightarrow \mathrm{H}^{j}(X / Z) \rightarrow \mathrm{H}^{j}(Y / Z) \stackrel{\partial}{\rightarrow} \mathrm{H}^{j+1}(X / Y) \rightarrow \mathrm{H}^{j+1}(X / Z) \rightarrow \ldots
$$

Proof. [Pri4] Proposition 1.61. 


\subsection{Model structures.}

Theorem 1.32. There is a simplicial model structure on scSp, for which the fibrations are quasi-smooth morphisms, and weak equivalences between quasi-smooth objects are those given in Definition 1.22.

Proof. This is [Pri4] Theorem 2.14.

Thus the homotopy category $\mathrm{Ho}(s c \mathrm{Sp})$ is equivalent to the category of quasi-smooth objects in $s c \mathrm{Sp}$, localised at the weak equivalences of Definition 1.22.

Definition 1.33. Given any morphism $f: X \rightarrow Z$, we define $\mathrm{H}^{n}(X / Z):=\mathrm{H}^{n}(\hat{X} / Z)$, for $X \stackrel{i}{\rightarrow} \hat{X} \stackrel{p}{\rightarrow} Z$ a factorisation of $f$ with $i$ a geometric trivial cofibration, and $p$ a geometric fibration.

\subsubsection{Homotopy representability.}

Definition 1.34. Define the category $\mathcal{S}$ to consist of functors $F: s \mathcal{C}_{\Lambda} \rightarrow \mathbb{S}$ satisfying the following conditions:

(A0) $F(k)$ is contractible.

(A1) For all small extensions $A \rightarrow B$ in $s \mathcal{C}_{\Lambda}$, and maps $C \rightarrow B$ in $s \mathcal{C}_{\Lambda}$, the map $F\left(A \times{ }_{B} C\right) \rightarrow F(A) \times{ }_{F(B)}^{h} F(C)$ is a weak equivalence, where $\times{ }^{h}$ denotes homotopy fibre product.

(A2) For all acyclic small extensions $A \rightarrow B$ in $s \mathcal{C}_{\Lambda}$, the map $F(A) \rightarrow F(B)$ is a weak equivalence.

Say that a natural transformation $\eta: F \rightarrow G$ between such functors is a weak equivalence if the maps $F(A) \rightarrow G(A)$ are weak equivalences for all $A \in s \mathcal{C}_{\Lambda}$, and let $\operatorname{Ho}(\mathcal{S})$ be the category obtained by formally inverting all weak equivalences in $\mathcal{S}$.

Theorem 1.35. There is a canonical equivalence between the geometric homotopy category $\mathrm{Ho}(s c \mathrm{Sp})$ and the category $\operatorname{Ho}(\mathcal{S})$.

Proof. This is [Pri4] Theorem 2.30.

1.6.2. Equivalent formulations. If $k$ is a field of characteristic 0, then we may work with dg algebras rather than simplicial algebras.

Definition 1.36. Define $d g \mathcal{C}_{\Lambda}$ to be the category of Artinian local differential $\mathbb{N}_{0}$-graded graded-commutative $\Lambda$-algebras with residue field $k$.

Definition 1.37. Define a map $A \rightarrow B$ in $d g \mathcal{C}_{\Lambda}$ to be a small extension if it is surjective and the kernel $I$ satisfies $I \cdot \mathfrak{m}(A)=0$.

Definition 1.38. Define $s D G$ Sp to be the category of left exact functors from $d g \mathcal{C}_{\Lambda}$ to $\mathbb{S}$.

Definition 1.39. Say a map $X \rightarrow Y$ in $s D G$ Sp is quasi-smooth if for all small extensions $f: A \rightarrow B$ in $d g \mathcal{C}_{\Lambda}$, the morphism

$$
X(A) \rightarrow Y(A) \times_{Y(B)} X(B)
$$

is a fibration in $\mathbb{S}$, which is moreover a trivial fibration if $f$ is acyclic.

Definition 1.40. We will say that a morphism $\alpha: F \rightarrow G$ of quasi-smooth objects of $s D G \mathrm{Sp}$ is a weak equivalence if, for all $A \in s \mathcal{C}_{\Lambda}$, the maps $\pi_{i} F(A) \rightarrow \pi_{i} G(A)$ are isomorphisms for all $i$.

Proposition 1.41. There is a model structure on sDGSp, for which the fibrations are quasi-smooth morphisms, and weak equivalences between quasi-smooth objects are those given in Definition 1.40 . 
Proof. This is [Pri4] Proposition 4.12.

Most of the constructions from $s \mathcal{C}_{\Lambda}$ carry over to $d g \mathcal{C}_{\Lambda}$. However, there is no straightforward analogue of Definition 1.15.

Definition 1.42. Define the normalisation functor $N: s \mathcal{C}_{\Lambda} \rightarrow d g \mathcal{C}_{\Lambda}$ by mapping $A$ to its associated normalised complex $N A$, equipped with the Eilenberg-Zilber shuffle product (as in [Qui]).

Definition 1.43. Define Spf $N^{*}: s D G \mathrm{Sp} \rightarrow s c \operatorname{Sp}$ by mapping $X: d g \mathcal{C}_{\Lambda} \rightarrow \mathbb{S}$ to the composition $X \circ N: s \mathcal{C}_{\Lambda} \rightarrow \mathbb{S}$. Note that this is well-defined, since $N$ is left exact.

Theorem 1.44. Spf $N^{*}: s D G \mathrm{Sp} \rightarrow s c \mathrm{Sp}$ is a right Quillen equivalence.

Proof. This is [Pri4] Theorem 4.18.

In particular, this means that $\operatorname{Spf} N^{*}$ maps quasi-smooth morphisms to quasi-smooth morphisms, and induces an equivalence $\mathbf{R S p f} N^{*}: \operatorname{Ho}(s D G \mathrm{Sp}) \rightarrow \operatorname{Ho}(s c \mathrm{Sp})$.

\section{DERIVED DEFORMATION COMPLEXES}

\subsection{Definitions.}

Definition 2.1. Define a pre-SDC to consist of homogeneous functors $E^{n}: \mathcal{C}_{\Lambda} \rightarrow$ Set, for $n \in \mathbb{N}_{0}$, together with maps

$$
\begin{array}{ll}
\partial^{i}: E^{n} \rightarrow E^{n+1} & 1 \leq i \leq n \\
\sigma^{i}: E^{n} \rightarrow E^{n-1} & 0 \leq i<n,
\end{array}
$$

an associative product $*: E^{m} \times E^{n} \rightarrow E^{m+n}$, with identity $1: \bullet \rightarrow E^{0}$, such that:

(1) $\partial^{j} \partial^{i}=\partial^{i} \partial^{j-1} \quad i<j$.

(2) $\sigma^{j} \sigma^{i}=\sigma^{i} \sigma^{j+1} \quad i \leq j$.

(3) $\sigma^{j} \partial^{i}=\left\{\begin{array}{cc}\partial^{i} \sigma^{j-1} & i<j \\ \operatorname{id} & i=j, i=j+1 \\ \partial^{i-1} \sigma^{j} & i>j+1\end{array}\right.$.

(4) $\partial^{i}(e) * f=\partial^{i}(e * f)$.

(5) $e * \partial^{i}(f)=\partial^{i+m}(e * f)$, for $e \in E^{m}$.

(6) $\sigma^{i}(e) * f=\sigma^{i}(e * f)$.

(7) $e * \sigma^{i}(f)=\sigma^{i+m}(e * f)$, for $e \in E^{m}$.

Remark 2.2. Note that a pre-SDC is an SDC (in the sense of [Pri2] if and only if the spaces $E^{n}$ are smooth for all $n$.

Definition 2.3. Define a pre-derived deformation complex (pre-DDC) $E$ to be a simplicial complex $E_{\bullet}$ of pre-SDCs.

Given $K \in \mathbb{S}$, observe that $E_{K}^{n}:=\operatorname{Hom}_{\mathbb{S}}\left(K, E^{n}\right)$ is a pre-SDC.

Remark 2.4. If each $E_{m}$ is an SDC, then Lemma 1.20 implies that for all $n, E^{n}: \mathcal{C}_{\Lambda} \rightarrow \mathbb{S}$ is smooth. For $K \in \mathbb{S}$ contractible, this implies that $E_{K}$ is an SDC.

Definition 2.5. Given a left-exact functor $F: \mathcal{C}_{\Lambda} \rightarrow$ Set, define the tangent space $t_{F}$ (or $t(F)$ ) by $t_{F}:=F\left(k[\epsilon] /\left(\epsilon^{2}\right)\right)$. Since $k[\epsilon] /\left(\epsilon^{2}\right)$ is an abelian group object in $\mathcal{C}_{\Lambda}, t_{F}$ is an abelian group. The endomorphisms $\epsilon \mapsto \lambda \epsilon$ of $k[\epsilon] /\left(\epsilon^{2}\right)$ make $t_{F}$ into a vector space over $k$.

Given a morphism $\alpha: F \rightarrow G$ of such functors, define the relative tangent space $\tan (F / G):=\operatorname{ker}(\tan F \rightarrow \tan G)$.

Definition 2.6. Given a morphism $f: E \rightarrow F$ of pre-SDCs for which each $f^{n}: E^{n} \rightarrow F^{n}$ is smooth, we may define cohomology groups $\mathrm{H}^{*}(E / F)$ as cohomology of the cosimplicial complex $\mathrm{C}^{\bullet}(E / F)$ given by $\mathrm{C}^{n}(E / F):=\tan (E / F)$, with cosimplicial structure defined as in $[$ Pri2] $\S 1$. 
Definition 2.7. Given a morphism $E \rightarrow F$ of pre-DDCs, levelwise smooth in the sense that each $f_{n}^{i}: E_{n}^{i} \rightarrow F_{n}^{i}$ is smooth, observe that the cohomology groups $\mathrm{H}^{i}(E / F)$ are simplicial vector spaces, and denote the corresponding normalised chain complexes by $N^{i}(E / F)$.

Definition 2.8. A morphism $f: E \rightarrow F$ of pre-DDCs is said to be quasi-smooth if:

Q1. for all $n, i \geq 0, E_{n}^{i} \rightarrow E_{\partial \Delta^{n}}^{i} \times_{F_{\partial \Delta^{n}}^{i}} F_{n}^{i}$ is smooth, and

Q2. for all $i>0, \mathrm{H}^{i}(E / F)$ is a constant simplicial complex, or equivalently

Q2'. for all $n>0, i>0, N_{n} \mathrm{H}^{i}(E / F)=0$.

Say that a pre-DDC $E$ is a DDC if it is quasi-smooth, i.e. if $E \rightarrow \bullet$ is quasi-smooth.

Definition 2.9. Given a levelwise smooth morphism $f: E \rightarrow F$ of pre-DDCs, define the tangent chain cochain complex by $N \mathrm{NC}_{\bullet}(E / F)$.

Definition 2.10. Say that a simplicial cosimplicial complex $V \in s c$ Vect $_{k}$ is quasi-smooth if $\mathrm{H}_{n}\left(N V^{i}\right)=0$ for all $n, i \geq 0$ and $\mathrm{H}^{i}(N V)_{n}=0$ for all $i>0$ and $n>0$.

Definition 2.11. Given $V \in s c$ Vect $_{k}$ quasi-smooth, define a cochain complex $\lrcorner V$ by:

$$
(\lrcorner V)^{n}:=\left\{\begin{array}{cc}
V_{0}^{n} & n \geq 0 \\
\mathrm{H}^{0}\left(N_{-n} V\right) & n<0,
\end{array}\right.
$$

with differential $d_{c}$ in non-negative degrees, and $d^{s}$ in negative degrees.

Given a levelwise smooth morphism $f: E \rightarrow F$ of pre-DDCs, define the cohomology groups $\left.\left.\mathrm{H}^{*}(\lrcorner E / F\right):=\mathrm{H}^{*}(\lrcorner \mathrm{C}^{\bullet}(E / F)\right)$, noting that these are given by

$$
\left.\mathrm{H}^{i}(\lrcorner E / F\right) \cong\left\{\begin{array}{cc}
\mathrm{H}^{i}\left(\mathrm{C}_{0}^{\bullet}(E / F)\right) & i>0 \\
\mathrm{H}_{-i} \mathrm{H}^{0}(N \mathrm{C} \bullet(E / F)) & i \leq 0 .
\end{array}\right.
$$

Lemma 2.12. If $V \in \operatorname{scVect}_{k}$ is quasi-smooth, then the inclusion map

$$
\lrcorner V \rightarrow \operatorname{Tot} N V
$$

is a quasi-isomorphism, and

$$
\left.\mathrm{H}_{i}\left(N Z^{n} V\right) \cong \mathrm{H}^{n-i}(\lrcorner V\right)
$$

for all $i, n \geq 0$.

Proof. Combine the proofs of [Pri4] Lemma 1.56 and [Pri4] Proposition 1.59.

Lemma 2.13. A levelwise smooth morphism $f: E \rightarrow F$ of pre-DDCs is quasi-smooth if and only if $\mathrm{C}:(E / F)$ is quasi-smooth (in the sense of Definition 2.10).

Proof. Since $f$ is levelwise smooth, we know by Proposition 1.20 that each $E^{i} \rightarrow F^{i}$ is a smooth map of functors $\mathcal{C}_{\Lambda} \rightarrow \mathbb{S}$. For a small extension $A \rightarrow B$ in $\mathcal{C}_{\Lambda}$ with kernel $I$, we thus deduce that $E^{i}(A) \rightarrow F^{i}(A) \times_{F^{i}(B)} E^{i}(B)$ is a fibration, with fibre $\mathrm{C}_{\bullet}^{i}(E / F) \otimes I$.

Hence $\mathrm{H}_{*} \mathrm{C}_{\bullet}^{i}(E / F)=0$ for all $i$ if and only if we have $E^{i} \rightarrow F^{i}$ trivially smooth for all $i$, i.e. if Definition 2.8.(Q1) holds. The result now follows from the characterisation of Definition 2.10 .

Definition 2.14. A morphism $f: E \rightarrow F$ of DDCs is said to be a quasi-isomorphism if $\left.\left.\left.\mathrm{H}^{*}(\lrcorner f\right): \mathrm{H}^{*}(\lrcorner E\right) \rightarrow \mathrm{H}^{*}(\lrcorner F\right)$ is an isomorphism.

Definition 2.15. Recall from [Pri5] Definition 4.1 that for any pre-SDC $E$, we define the Maurer-Cartan functor $\mathrm{MC}_{E}: s \mathcal{C}_{\Lambda} \rightarrow$ Set by

$$
\operatorname{MC}_{E}(A) \subset \prod_{n \geq 0} E^{n+1}\left(A^{I^{n}}\right)
$$


consisting of those $\underline{\omega}$ satisfying:

$$
\begin{aligned}
\omega_{m}\left(s_{1}, \ldots, s_{m}\right) * \omega_{n}\left(t_{1}, \ldots, t_{n}\right) & =\omega_{m+n+1}\left(s_{1}, \ldots, s_{m}, 0, t_{1}, \ldots, t_{n}\right) ; \\
\partial^{i} \omega_{n}\left(t_{1}, \ldots, t_{n}\right) & =\omega_{n+1}\left(t_{1}, \ldots, t_{i-1}, 1, t_{i}, \ldots, t_{n}\right) ; \\
\sigma^{i} \omega_{n}\left(t_{1}, \ldots, t_{n}\right) & =\omega_{n-1}\left(t_{1}, \ldots, t_{i-1}, \min \left\{t_{i}, t_{i+1}\right\}, t_{i+2}, \ldots, t_{n}\right) ; \\
\sigma^{0} \omega_{n}\left(t_{1}, \ldots, t_{n}\right) & =\omega_{n-1}\left(t_{2}, \ldots, t_{n}\right) ; \\
\sigma^{n-1} \omega_{n}\left(t_{1}, \ldots, t_{n}\right) & =\omega_{n-1}\left(t_{1}, \ldots, t_{n-1}\right), \\
\sigma^{0} \omega_{0} & =1,
\end{aligned}
$$

where $I:=\Delta^{1}$.

Definition 2.16. Given a pre-DDC $E$, define the derived Maurer-Cartan functor $\mathfrak{M C}(E)$ : $s \mathcal{C}_{\Lambda} \rightarrow \mathbb{S}$ by $\operatorname{Hom}_{\mathbb{S}}(K, \mathfrak{M C}(E)):=\operatorname{MC}\left(E_{K}\right)$.

Proposition 2.17. If $f: E \rightarrow F$ is a quasi-smooth morphism of pre-DDCs, then

$$
\mathfrak{M C}(f): \mathfrak{M C}(E) \rightarrow \mathfrak{M C}(F)
$$

is quasi-smooth, with cohomology groups

$$
\left.\mathrm{H}^{i}(\mathfrak{M C}(E) / \mathfrak{M C C}(F)) \cong \mathrm{H}^{i+1}(\lrcorner E / F\right) .
$$

In particular, if $E$ is a $D D C$, then $\mathfrak{M C}(E)$ is quasi-smooth.

Proof. By construction, the simplicial matching maps are given by

$$
\mathrm{MC}\left(E_{n}\right) \rightarrow \mathrm{MC}\left(F_{n}\right) \times_{\mathrm{MC}\left(F_{\partial \Delta^{n}}\right)} \mathrm{MC}\left(E_{\partial \Delta^{n}}\right)=\mathrm{MC}\left(E_{\partial \Delta^{n}} \times_{F_{\partial \Delta^{n}}} F_{n}\right) .
$$

Condition (Q1) from Definition 2.8 for $f$ implies that

$$
E_{n} \rightarrow E_{\partial \Delta^{n}} \times_{F_{\partial \Delta^{n}}} F_{n}
$$

is a levelwise smooth map of SDCs, so [Pri5] Proposition 4.3 implies that $\mathfrak{M C}(f)$ satisfies condition (S1) from Definition 1.13.

We now need to check that the quasi-smooth partial matching maps

$$
\alpha: \operatorname{MC}\left(E_{n}\right) \rightarrow \operatorname{MC}\left(E_{\Lambda_{k}^{n}}\right) \times_{\operatorname{MC}\left(F_{\Lambda_{k}^{n}}\right)} \operatorname{MC}\left(F_{n}\right)
$$

are smooth. To do this, we verify the criterion of Corollary 1.30.

Taking the relative version of [Pri5] Proposition 4.7, we see that $\mathrm{H}^{*}(\alpha)$ is cohomology of the cochain complex

$$
\operatorname{ker}\left(N_{c}\left(E_{n} / F_{n}\right)[1] \rightarrow N_{c}\left(E_{\Lambda_{k}^{n}} / F_{\Lambda_{k}^{n}}\right) ;\right.
$$

this is isomorphic to $N_{n}^{s} N_{c}(E / F)[1]$, which gives isomorphisms

$$
\mathrm{H}^{0}(\alpha) \cong N_{n} \mathrm{Z}^{1} \mathrm{C}(E / F), \quad \mathrm{H}^{i}(\alpha) \cong N_{n} \mathrm{H}^{i+1}(E / F) \text { for } i>0 .
$$

Since $N_{n} \mathrm{H}^{i}(E / F)=0$ all $i>0$ by condition (Q2'), we see that $\mathrm{H}^{i}(\alpha)=0$ for all $i>0$. This implies quasi-smoothness of $\mathfrak{M C}(f)$.

Now for $i>0$, the calculations above combine with Lemma 2.12 to give

$$
\left.\mathrm{H}^{i}(\mathfrak{M C}(E) / \mathfrak{M C}(F))=\mathrm{H}^{i}\left(\mathrm{MC}\left(E_{0}\right) / \mathrm{MC}\left(F_{0}\right)\right)=\mathrm{H}^{i+1}\left(E_{0} / F_{0}\right)=\mathrm{H}^{i+1}(\lrcorner E / F\right) .
$$

For $i \leq 0$,

$$
\left.\mathrm{H}^{i}(\mathfrak{M C}(E) / \mathfrak{M C}(F))=\mathrm{H}_{-i} \mathrm{H}^{0}(\mathrm{C}(E / F)[1])=\mathrm{H}_{-i} \mathrm{Z}^{1}(\mathrm{C}(E / F))=\mathrm{H}^{i+1}(\lrcorner E / F\right),
$$

since $\mathrm{C}(E / F)$ is quasi-smooth (in the sense of Definition 2.10)

Corollary 2.18. If $f: E \rightarrow F$ is a quasi-isomorphism of DDCs, then $\mathfrak{M C}(f): \mathfrak{M C}(E) \rightarrow$ $\mathfrak{M C}(F)$ is a weak equivalence. 
Definition 2.19. Given a pre-DDC $E$, note that $E^{0}$ acts on $\mathfrak{M C}(E)$ by conjugation. Define the derived deformation functor $\mathfrak{D e f}(E): s \mathcal{C}_{\Lambda} \rightarrow \mathbb{S}$ by

$$
\mathfrak{D e f}(E):=\left[\mathfrak{M C}(E) / E^{0}\right],
$$

the homotopy quotient (as in Definition 1.23).

Lemma 2.20. Given a simplicial group $G$, and a fibration $X \rightarrow Y$ of simplicial $G$-sets, if each $X_{n}$ and $Y_{n}$ is a free $G_{n}$-set, then $X / G \rightarrow Y / G$ is a fibration in $\mathbb{S}$.

Proof. Combine [GJ] Corollary V.2.7 and [GJ] Lemma V.3.7.

Corollary 2.21. If $f: E \rightarrow F$ is a quasi-smooth morphism of pre-DDCs, then

$$
(f, q): \mathfrak{D e f}(E) \rightarrow \mathfrak{D} \mathfrak{e f}(F) \times_{\bar{W} F^{0}} \bar{W} E^{0},
$$

is quasi-smooth, where $\bar{W} G:=G \backslash W G$ is a model for the classifying space BG of $G$ (as in [GJ] $\S V .4)$.

Thus

is quasi-smooth, and

$$
\mathfrak{D e f}(f): \mathfrak{D} \mathfrak{e f}(E) \rightarrow \mathfrak{D e f}(F)
$$

$$
\mathfrak{M C}(E) \rightarrow \mathfrak{D} \mathfrak{e f}(E) \times_{\mathfrak{D} \mathfrak{f}(F)} \mathfrak{M C C}(F)
$$

is a weak equivalence.

In particular, if $E$ is a $D D C$, then $\mathfrak{M C}(E) \rightarrow \mathfrak{D e f}(E)$ is a weak equivalence.

Proof. First observe that $E^{0} \rightarrow F^{0}$ is trivially smooth, so $\mathfrak{M C}(E) \times W E^{0} \rightarrow \mathfrak{M C}(F) \times W E^{0}$ is quasi-smooth.

Given a surjection $A \rightarrow B$ in $s \mathcal{C}_{\Lambda}$, apply Lemma 2.20, taking

$$
X=W E^{0}(A) \times \mathfrak{M C}(E)(A), \quad Y=W E^{0}(A) \times \mathfrak{M C}(E)(B) \times_{\mathfrak{M C}(F)(B)} \mathfrak{M C}(F)(A),
$$

and $G=E^{0}(A)$. This shows that $(f, q)$ satisfies condition (S1) from Definition 1.13. Condition (Q2) follows similarly, so $(f, q)$ is quasi-smooth.

That $\mathfrak{D e f}(F)$ is quasi-smooth follows from the observation that $\bar{W} E^{0} \rightarrow \bar{W} F^{0}$ is trivially smooth.

For the final statements, note that $\mathfrak{M C C}(E)=\mathfrak{D e f}(E) \times_{\bar{W}} E^{0} 1$, and

$$
Y:=\mathfrak{D e f}(E) \times_{\mathfrak{D} \mathfrak{e f}(F)} \mathfrak{M} \mathfrak{C}(F)=\mathfrak{D e f}(E) \times_{\bar{W} F^{0}} 1
$$

If $Z:=\bar{W} E^{0} \times_{\bar{W} F^{0}} 1$, then $Z$ is trivially fibrant, so $1 \rightarrow Z$ is a weak equivalence. The map $\mathfrak{M C}(E) \rightarrow Y$ is then just the pullback of $1 \rightarrow Z$ along $Y \rightarrow Z$.

\subsection{Comparison with SDCs.}

Definition 2.22. Given a pre-DDC $E$, define a pre-DDC $D E$ by $(D E)_{n}:=\left(E_{n}\right)^{\Delta^{n}}$, in the notation of [Pri5] Definition 3.11, i.e.

$$
\left(E^{X}\right)^{n}=\left(E^{n}\right)^{X_{n}} .
$$

For $x \in X_{n+1}, y \in Y_{n+1}, z \in X_{m+n}, 1 \leq i \leq n, 0 \leq j<n, e \in\left(E^{X}\right)^{n}$ and $f \in\left(E^{X}\right)^{m}$, we define the operations by

$$
\begin{aligned}
\partial^{i}(e)(x) & :=\partial^{i}\left(e\left(\partial_{i} x\right)\right) \\
\sigma^{j}(e)(y) & :=\sigma^{j}\left(e\left(\sigma_{i} y\right)\right), \\
(f * e)(z) & :=f\left(\left(\partial_{m+1}\right)^{n} z\right) * e\left(\left(\partial_{0}\right)^{m} z\right) .
\end{aligned}
$$

Proposition 2.23. If $f: E \rightarrow F$ is a map of pre-DDCs with

(1) $f^{i}: E^{i} \rightarrow F^{i}$ smooth for all $i$, and

(2) $\mathrm{H}^{i}(E / F)$ a constant simplicial complex for all $i>0$, then $D f: D E \rightarrow D F$ is quasi-smooth.

In particular, $D E$ is a $D D C$ for all SDCs $E$. 
Proof. By smooth base change, we know that $D f$ is levelwise smooth. We now verify the conditions of Lemma 2.13.

$$
\mathrm{C}^{i}(D E / D F)_{n}=\mathrm{C}^{i}\left(E_{n} / F_{n}\right)^{\Delta_{i}^{n}}=\mathrm{C}^{i}\left(E_{n} / F_{n}\right) \otimes \mathrm{C}^{i}\left(\Delta^{n}, k\right) .
$$

Thus

$$
\mathrm{H}_{*}(D E / D F)^{i}=\mathrm{H}_{*}\left(E^{i} / F^{i}\right) \otimes \mathrm{H}_{*}\left(\mathrm{C}^{i}\left(\Delta^{\bullet}, k\right)\right)=0,
$$

since the cosimplicial complex $k \otimes \Delta_{i}^{\bullet}$ is contractible. Moreover,

$$
\mathrm{H}^{*}(D E / D F)_{n}=\mathrm{H}^{*}\left(E_{n} / F_{n}\right) \otimes \mathrm{H}^{*}\left(\Delta^{n}, k\right)=\mathrm{H}^{*}\left(E_{n} / F_{n}\right),
$$

so $\mathrm{H}^{i}(D E / D F)_{n}$ is constant for $i>0$.

Lemma 2.24. If $X$ in scSp is a levelwise quasi-smooth object for which the simplicial vector spaces $\mathrm{H}^{i}(\tan X)$ have constant simplicial structure for $i>0$, then

$$
\mathrm{H}^{*}(X) \cong \mathrm{H}^{*}(\operatorname{Tot} N \tan X),
$$

as defined in Definitions 1.33 and 2.5 respectively.

Proof. By [Pri4] Lemma 2.26, there is a weak equivalence $X \rightarrow \underline{X}$ to a quasi-smooth object. Since this is also a levelwise weak equivalence, we find that $\operatorname{Tot} N \tan X \rightarrow \operatorname{Tot} N \tan \underline{X}$ is a quasi-isomorphism. Now Proposition 1.21 implies that $\underline{X}$ is quasi-smooth, so $\mathrm{H}^{*}(X) \cong$ $\mathrm{H}^{*}(\underline{X}) \cong \mathrm{H}^{*}(\operatorname{Tot} N \tan X)$, the last isomorphism coming from [Pri4] Theorem 1.59.

Proposition 2.25. If $E$ is a pre-DDC for which $E \rightarrow \bullet$ satisfies the conditions of Proposition 2.23, then $\alpha: \mathfrak{D} \mathfrak{e f}(E) \rightarrow \mathfrak{D} \mathfrak{e f}(D E)$ is a quasi-smooth replacement for $\mathfrak{D} \mathfrak{e f}(E)$.

Proof. By Proposition 2.23, we know that $\mathfrak{D e f}(D E)$ is quasi-smooth, so we just need to show that $\alpha$ is a weak equivalence. Now, for $i>0$,

$$
\mathrm{H}^{i}(\tan \mathfrak{D} \mathfrak{e f}(E))=H^{i}(\tan \mathfrak{M C}(E))=\mathrm{H}^{i+1}(E),
$$

which has constant simplicial structure, so $\mathrm{H}^{i}(\mathfrak{D e f}(E))=\mathrm{H}^{i}(\operatorname{Tot} N \tan \mathfrak{D} \mathfrak{e f}(E))=$ $\mathrm{H}^{i+1}\left(\operatorname{Tot} N \mathrm{C}^{\bullet}(E)\right)$.

Similarly, $\mathrm{H}^{i}(\mathfrak{D e f}(D E))=\mathrm{H}^{i+1}\left(\operatorname{Tot} N \mathrm{C}^{\bullet}(D E)\right) \cong \mathrm{H}^{i+1}\left(\operatorname{Tot} N \mathrm{C}^{\bullet}(E)\right)$, so [Pri4] Corollary 2.16 ensures that $\alpha$ is a weak equivalence.

Corollary 2.26. For an $S D C E$, the functors $\operatorname{Def}_{E}$ (from [Pri5] Definition 4.4) and $\mathfrak{D e f}(D E)$ (and hence $\mathfrak{M C}(D E)$ ) are weakly equivalent (in scSp).

Proof. Recall that $\operatorname{Def}_{E}$ is $\left[\underline{\mathrm{MC}}_{E} / \underline{E}^{0}\right]$, which is a quasi-smooth replacement of $\left[\mathrm{MC}_{E} / E^{0}\right]$ by [Pri4] Lemma 2.26, in the sense that there is a weak equivalence $\left[\mathrm{MC}_{E} / E^{0}\right] \rightarrow \operatorname{Def}_{E}$. If we let $E$ denote the constant pre-DDC $E_{n}:=E$, then $\left[\mathrm{MC}_{E} / E^{0}\right]=\mathfrak{D e f}(E)$, and we may apply Proposition 2.25.

Lemma 2.27. If $E$ is a pre-DDC for which $E \rightarrow \bullet$ satisfies the conditions of Proposition 2.23, then for all $A \in \mathcal{C}_{\Lambda}$, the map $\alpha(A): \mathfrak{D e f}(E)(A) \rightarrow \mathfrak{D e f}(D E)(A)$ is a weak equivalence in $\mathbb{S}$.

Proof. First observe that $\mathfrak{D e f}(E)(A)_{0}=\operatorname{MC}_{E_{0}}(A)=\mathfrak{D} \mathfrak{e f}(D E)(A)$, and write $\pi^{0} F:=$ $\left.F\right|_{\mathcal{C}_{\Lambda}}$. Now, $\tan \pi^{0} \mathfrak{D} \mathfrak{e f}(E)$ is the mapping cone of $\mathrm{C}^{0}(E) \stackrel{d_{c}}{\longrightarrow} \mathrm{Z}^{1}(E)$, so (Q2) ensures that $\left.\pi_{n} \tan \pi^{0} \mathfrak{D} \mathfrak{e f}(E)=\mathrm{H}^{1-n}(\lrcorner E\right)$. Observe that $\left.\pi_{n} \tan \pi^{0} \mathfrak{D} \mathfrak{e f}(D E)=\mathrm{H}^{1-n}(\lrcorner E\right)$, similarly.

The proofs of Proposition 1.28 and Corollary 1.29 adapt to show that $\alpha(A)$ is a weak equivalence in $\mathbb{S}$ for all $A$, by taking small extensions $A \rightarrow B$ with kernel $I$, and considering the long exact sequences

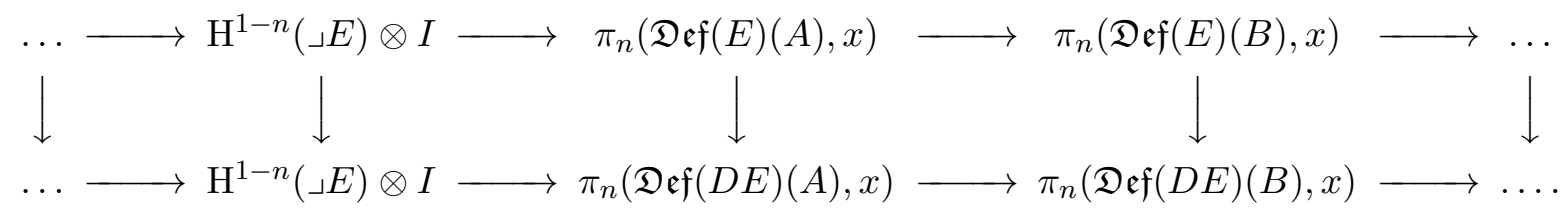


associated to the fibrations $\mathfrak{D e f}(E)(A) \rightarrow \mathfrak{D e f}(E)(B)$ and $\mathfrak{D e f}(D E)(A) \rightarrow \mathfrak{D} \mathfrak{e f}(D E)(B)$.

\section{Constructing DDCs}

3.1. Simplicial monadic adjunctions. A simplicial category $\mathcal{C}$ has a class $\mathrm{Ob} \mathcal{C}$ of objects, and for all $A, B \in \mathrm{Ob} \mathcal{C}$, a simplicial set $\underline{\operatorname{Hom}}_{\mathcal{C}}(A, B)$ of morphisms, with the usual multiplication and identity properties. For a simplicial category $\mathcal{C}$, we denote by $\mathcal{C}_{n}$ the category with objects $\operatorname{Ob} \mathcal{C}$ and morphisms $\operatorname{Hom}_{\mathcal{C}_{n}}(A, B):=\underline{\operatorname{Hom}}_{\mathcal{C}}(A, B)_{n}$.

Definition 3.1. Say that a functor $F: \mathcal{C} \rightarrow \mathcal{D}$ of simplicial categories is an equivalence if the functors $F_{n}: \mathcal{C}_{n} \rightarrow \mathcal{D}_{n}$ are all equivalences.

Definition 3.2. Given a simplicial category $\mathcal{C}$, set $\operatorname{Hom}_{\mathcal{C}}(A, B):=\left(\underline{\operatorname{Hom}}_{\mathcal{C}}\right)_{0}(A, B)$.

Definition 3.3. For simplicial categories $\mathcal{D}, \mathcal{E}$, and a pair of functors

$$
\mathcal{D} \underset{F}{\stackrel{G}{\rightleftarrows}} \mathcal{E},
$$

recall that an adjunction $F \dashv G$ is a natural isomorphism

$$
\underline{\operatorname{Hom}}_{\mathcal{D}}(F A, B) \cong \underline{\operatorname{Hom}}_{\mathcal{E}}(A, G B) \text {. }
$$

We say that $F$ is left adjoint to $G$, or $G$ is right adjoint to $F$. Let $\perp=F G$, and $\top=G F$. To give an adjunction is equivalent to giving two natural transformations, the unit and co-unit

$$
\eta: \operatorname{id}_{\mathcal{E}} \rightarrow \top, \quad \varepsilon: \perp \rightarrow \operatorname{id}_{\mathcal{D}}
$$

satisfying the triangle identities $\varepsilon F \circ F \eta=\mathrm{id}_{F}, G \varepsilon \circ \eta G=\mathrm{id}_{G}$.

Given an adjunction

$$
\mathcal{D} \underset{F}{\stackrel{U}{\underset{F}{\longrightarrow}} \mathcal{E}}
$$

with unit $\eta:$ id $\rightarrow U F$ and co-unit $\varepsilon: F U \rightarrow$ id, we let $\top=U F$, and define the simplicial category $\mathcal{E}^{\top}$ of $\top$-algebras to have objects

$$
\top E \stackrel{\theta}{\rightarrow} E,
$$

for $\theta \in \underline{\operatorname{Hom}}_{0}(\top E, E)$, such that $\theta \circ \eta_{E}=\mathrm{id}$ and $\theta \circ \top \theta=\theta \circ U \varepsilon_{F E}$. We define morphisms by setting

$$
\underline{\operatorname{Hom}}_{\mathcal{E}^{\top}}\left(\top E_{1} \stackrel{\theta}{\rightarrow} E_{1}, \top E_{2} \stackrel{\phi}{\rightarrow} E_{2}\right) \subset \underline{\operatorname{Hom}}_{\mathcal{E}}\left(E_{1}, E_{2}\right)
$$

to be the equaliser of

$$
\underline{\operatorname{Hom}}_{\mathcal{E}}\left(E_{1}, E_{2}\right) \underset{\theta^{*}}{\stackrel{\phi_{*} \circ \top}{\longrightarrow}} \operatorname{Hom}_{\mathcal{E}}\left(T E_{1}, E_{2}\right) .
$$

We define the comparison functor $K: \mathcal{D} \rightarrow \mathcal{E}^{\top}$ by

$$
B \mapsto\left(U F U B \stackrel{U \varepsilon_{B}}{\longrightarrow} U B\right)
$$

on objects, and $K(g)=U(g)$ on morphisms.

Definition 3.4. An adjunction

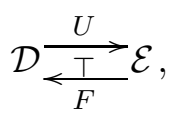

of simplicial categories is said to be monadic if $K: \mathcal{D} \rightarrow \mathcal{E}^{\top}$ is an equivalence. 
Examples 3.5. Intuitively, monadic adjunctions correspond to algebraic theories, such as the adjunction

$$
\operatorname{Ring} \underset{\mathbb{Z}[-]}{\stackrel{U}{\longrightarrow}} \text { Set, }
$$

between rings and sets, $U$ being the forgetful functor. Other examples are $k$-algebras over $k$-vector spaces, or groups over sets.

Definition 3.6. Given an adjunction

$$
\mathcal{D} \underset{G}{\stackrel{V}{\perp} \mathcal{E}}
$$

let $\perp=V G$, so $\perp$ opp is a monad on $\mathcal{E}^{\text {opp }}$. Define $\mathcal{E}_{\perp}:=\left(\left(\mathcal{E}^{\text {opp }}\right)^{\perp \text { opp }}\right)^{\text {opp }}$, with $K=K^{\text {opp }}$ : $\mathcal{D} \rightarrow \mathcal{E}_{\perp}$. The adjunction is said to be comonadic if $K: \mathcal{D} \rightarrow \mathcal{E}_{\perp}$ is an equivalence.

Example 3.7. If $X$ is a topological space (or any site with enough points) and $X^{\prime}$ is the set of points of $X$, let $u: X^{\prime} \rightarrow X$ be the associated morphism. Then the adjunction $u^{-1} \dashv u_{*}$ on sheaves is comonadic, so the category of sheaves on $X$ is equivalent $u^{-1} u_{*}$-coalgebras in the category of sheaves (or equivalently presheaves) on $X^{\prime}$

A more prosaic example is that for any ring $A$, the category of $A$-coalgebras is comonadic over the category of $A$-modules.

3.1.1. Bialgebras. As in [Van] $\S \mathrm{IV}$, take a category $\mathcal{B}$ equipped with both a monad $(\top, \mu, \eta)$ and a comonad $(\perp, \Delta, \gamma)$, together with a distributivity transformation $\lambda: \top \perp \Longrightarrow \perp \top$ satisfying various additional conditions.

Definition 3.8. Given a distributive monad-comonad pair $(\top, \perp)$ on a simplicial category $\mathcal{B}$, define the category $\mathcal{B}_{\perp}^{\top}$ of bialgebras as follows. The objects of $\mathcal{B}_{\perp}^{\top}$ are triples $(\theta, B, \beta)$ with $(\top B \stackrel{\theta}{\rightarrow} B)$ an object of $\mathcal{B}^{\top}$ and $B \stackrel{\beta}{\rightarrow} \perp B$ an object of $\mathcal{B}_{\perp}$, such that the composition $(\beta \circ \theta): \top B \rightarrow \perp B$ agrees with the composition

$$
\top B \stackrel{\top \beta}{\longrightarrow} \top \perp B \stackrel{\lambda}{\rightarrow} \perp \top B \stackrel{\perp \theta}{\longrightarrow} \perp B .
$$

Morphisms are then given by setting

$$
\underline{\operatorname{Hom}}_{\mathcal{B}}{ }_{\perp}\left(\top B \stackrel{\theta}{\rightarrow} B \stackrel{\beta}{\rightarrow} \perp B, \top B^{\prime} \stackrel{\theta^{\prime}}{\rightarrow} B^{\prime} \stackrel{\beta^{\prime}}{\rightarrow} \perp B^{\prime}\right) \subset \underline{\operatorname{Hom}}_{\mathcal{B}}\left(B, B^{\prime}\right)
$$

to be the equaliser of

$$
\underline{\operatorname{Hom}}_{\mathcal{B}}\left(B, B^{\prime}\right) \underset{\left(\theta^{*}, \beta^{*} \circ \perp\right)}{\stackrel{\left(\theta_{*}^{\prime} \circ \top, \beta_{*}^{\prime}\right)}{\longrightarrow}} \underline{\operatorname{Hom}}_{\mathcal{B}}\left(\top B, B^{\prime}\right) \times \underline{\operatorname{Hom}}_{\mathcal{B}}\left(B, \perp B^{\prime}\right) .
$$

Example 3.9. If $X$ is a topological space (or any site with enough points) and $X^{\prime}$ is the set of points of $X$, let $\mathcal{D}$ be the category of sheaves of rings on $X$. If $\mathcal{B}$ is the category of sheaves (or equivalently presheaves) of sets on $X^{\prime}$, then the description above characterises $\mathcal{D}$ as a category of bialgebras over $\mathcal{B}$, with the comonad being $u^{-1} u_{*}$ for $u: X^{\prime} \rightarrow X$, and the monad being the free polynomial functor.

3.2. The construction. We let $s$ Cat denote the category of simplicial categories.

Definition 3.10. Given functors $\mathcal{A} \stackrel{f}{\rightarrow} \mathcal{B} \stackrel{g}{\leftarrow} \mathcal{C}$ of simplicial categories, define the fibre product $\mathcal{A} \times{ }_{\mathcal{B}} \mathcal{C}$ by

$$
\mathrm{Ob}\left(\mathcal{A} \times{ }_{\mathcal{B}} \mathcal{C}\right)=\left\{(A, \beta, C): A \in \mathrm{Ob} \mathcal{A}, C \in \mathrm{Ob} C, \beta \in \mathrm{Iso}_{\mathcal{B}_{0}}(f A, g C)\right\},
$$

with morphisms

$$
\underline{\operatorname{Hom}}_{\mathcal{A} \times{ }_{\mathcal{B}} \mathcal{C}}\left((A, \beta, C),\left(A^{\prime}, \beta^{\prime}, C^{\prime}\right)\right)=\underline{\operatorname{Hom}}_{\mathcal{A}}\left(A, A^{\prime}\right) \times_{\beta_{*}^{\prime} f, \underline{\operatorname{Hom}}_{\mathcal{B}}\left(f A, g C^{\prime}\right), \beta^{*} g} \underline{\operatorname{Hom}}_{\mathcal{C}}\left(C, C^{\prime}\right) .
$$


Definition 3.11. We say that a functor $F: \mathcal{C}_{\Lambda} \rightarrow$ Set is homogeneous if for all small extensions $A \rightarrow B$ in $\mathcal{C}_{\Lambda}$,

$$
F\left(A \times{ }_{B} C\right) \rightarrow F(A) \times{ }_{F(B)} F(C)
$$

is an isomorphism. Note that this is equivalent to being a disjoint union of left-exact functors.

Similarly, a functor $\mathcal{D}: \mathcal{C}_{\Lambda} \rightarrow s$ Cat is said to be homogeneous if

$$
F\left(A \times{ }_{B} C\right) \rightarrow F(A) \times{ }_{F(B)} F(C)
$$

is an equivalence for all small extensions $A \rightarrow B$.

Definition 3.12. We say that a homogeneous functor $\mathcal{B}: \mathcal{C}_{\Lambda} \rightarrow s$ Cat has uniformly trivial deformation theory if

(1) for all $A \in \mathcal{C}_{\Lambda}$ and all $B_{1}, B_{2} \in \mathrm{Ob} \mathcal{B}(A)$, the functor $\underline{\operatorname{Hom}}_{\mathcal{B}}\left(B_{1}, B_{2}\right): \mathcal{C}_{A} \rightarrow$ Set of morphisms from $B_{1}$ to $B_{2}$ is trivially smooth (in the sense of Definition 1.19) and homogeneous;

(2) for $A^{\prime} \rightarrow A$ in $\mathcal{C}_{\Lambda}, \mathcal{B}_{0}\left(A^{\prime}\right) \rightarrow \mathcal{B}_{0}(A)$ is essentially surjective.

Now, assume that we have a diagram

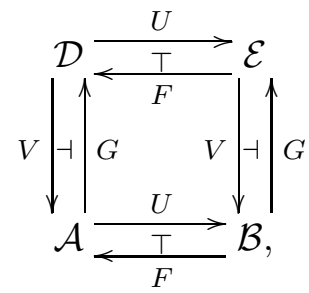

of adjunctions of homogeneous simplicial category-valued functors on $\mathcal{C}_{\Lambda}$, with $F \dashv U$ monadic and $G \vdash V$ comonadic. Let

$$
\begin{array}{ll}
\top_{\mathrm{h}}=U F & \perp_{\mathrm{h}}=F U \\
\perp_{\mathrm{v}}=V G & \top_{\mathrm{v}}=G V,
\end{array}
$$

with

$$
\eta: 1 \rightarrow \top_{\mathrm{h}}, \quad \gamma: \perp_{\mathrm{v}} \rightarrow 1, \quad \varepsilon: \perp_{\mathrm{h}} \rightarrow 1 \text { and } \alpha: 1 \rightarrow \top_{\mathrm{v}} .
$$

Assume that these adjunctions satisfy the simplicial analogues of [Van] $\S$ IV or [Pri2] $\S 2$, in other words that $U$ and $V$ commute with everything (although $G$ and $F$ need not commute).

Fix $D \in \mathrm{Ob} \mathcal{D}(k)$, such that we may lift $U V D \in \mathrm{Ob} \mathcal{B}(k)$ to $B \in \mathrm{Ob} \mathcal{B}(\Lambda)$, up to isomorphism (in $\mathcal{B}_{0}(k)$ ).

Theorem 3.13. There is a natural pre-DDC E associated to this diagram, given by

$$
E^{n}=\underline{\operatorname{Hom}}_{\mathcal{B}}\left(\top_{\mathrm{h}}^{n} B, \perp_{\mathrm{v}}^{n} B\right)_{U V\left(\alpha_{D}^{n} \circ \varepsilon_{D}^{n}\right)} .
$$

If $E$ is levelwise smooth, satisfying Condition (Q2) of Definition 2.8, then the classifying space $\bar{W} \mathcal{D}_{D, \text { id }}$ is canonically weakly equivalent to the restriction $\pi^{0} \mathfrak{D} \mathfrak{e f}(E)$ (from Lemma 2.27) as a functor from $\mathcal{C}_{\Lambda}$ to $\mathbb{S}$. Here $\mathcal{D}_{D, \text { id }}(A)$ is the simplicial groupoid given by the fibre product

$$
\mathcal{D}(A) \times_{\mathcal{D}(k)}(D, \mathrm{id}),
$$

where $(D, \mathrm{id})$ is the category with one object and one morphism.

Proof. For each $m, E_{m}$ is the SDC defined in [Pri2] $\S 2$ associated to the monad $\mathrm{T}_{\mathrm{h}}$ and comonad $\perp_{\mathrm{v}}$ over the category $\mathcal{B}_{m}$. 
Since the adjunctions are monadic or comonadic, the proof of [Pri2] Theorem 2.2 adapts to give functorial equivalences

$$
K(A): \mathcal{D}(A) \rightarrow \mathcal{B}_{\perp_{\mathrm{v}}}^{\top_{\mathrm{h}}}(A)
$$

between $\mathcal{D}$ and the simplicial category of $\left(T_{\mathrm{h}}, \perp_{\mathrm{v}}\right)$-bialgebras.

Let $D^{\prime} \in \mathrm{Ob}_{\perp_{\mathrm{v}}}^{\top_{\mathrm{h}}}(k)$ be the bialgebra over $\bar{B} \in \mathrm{Ob} \mathcal{B}(k)$, with bialgebraic structure coming from the isomorphism $U V D \cong \bar{B}$. Let $\mathcal{G}$ be the full subcategory of $\mathcal{B}_{\perp_{\mathrm{v}}}^{\top_{\mathrm{h}}}(A)$ on objects

$$
\mathrm{MC}_{E_{0}}(A)=\left\{\omega \in \mathrm{ObB}^{\top_{\mathrm{h}}, \perp_{\mathrm{v}}}(A): \bar{X}=D^{\prime} \in \mathrm{ObB}^{\top_{\mathrm{h}}, \perp_{\mathrm{v}}}(k)\right.
$$

Morphisms in $\mathcal{G}$ are just

$$
\underline{\operatorname{Hom}}_{\mathcal{G}}\left(\omega, \omega^{\prime}\right)=\left\{f \in E^{0}: f * \omega=\omega^{\prime} * f\right\},
$$

from which we deduce that $\mathcal{G}$ is a simplicial groupoid. Moreover, observe that $\mathcal{G} \rightarrow$ $\left(\mathcal{B}_{\perp_{\mathrm{v}}}^{\top_{\mathrm{h}}}\right)_{D^{\prime} \text {,id }}$ is an isomorphism of simplicial categories, so $\mathcal{G}$ is equivalent to $\mathcal{D}_{D \text {,id. }}$. In particular, this implies that $\mathcal{D}_{D \text {,id }}$ is a simplicial groupoid. It therefore suffices to compare $\mathcal{G}$ with $\mathfrak{D} \mathfrak{e f}(E)$.

Lemma 3.14. The functor $\mathcal{G}: \mathcal{C}_{\Lambda} \rightarrow s \mathrm{Gpd}$ is quasi-smooth, in the sense that it maps small extensions to fibrations (as defined in [GJ] $\S V .7$ ).

Proof of lemma. Smoothness of $E_{0}^{0}$ implies that the path-lifting property is satisfied. Given $K \hookrightarrow L \in \mathbb{S}$, and a small extension $A \rightarrow B$ with kernel $I$, the obstruction to lifting the diagram

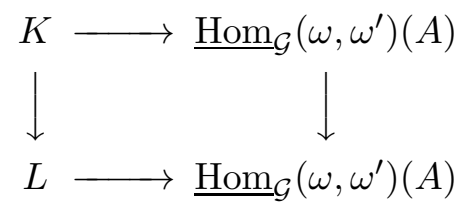

lies in $\mathrm{H}^{1}\left(\operatorname{ker}\left(\mathrm{C}^{\bullet}\left(E_{L}\right) \rightarrow \mathrm{C}^{\bullet}\left(E_{K}\right)\right)\right)$. If we write $V^{\bullet}=\operatorname{ker}\left(\mathrm{C}^{\bullet}\left(E_{L}\right) \rightarrow \mathrm{C}^{\bullet}\left(E_{K}\right)\right)$, then we have an exact sequence

$$
\mathrm{H}^{0}\left(E_{L}\right) \stackrel{\alpha}{\rightarrow} \mathrm{H}^{0}\left(E_{K}\right) \rightarrow \mathrm{H}^{1}\left(V^{\bullet}\right) \rightarrow \mathrm{H}^{1}\left(E_{L}\right) \stackrel{\beta}{\rightarrow} \mathrm{H}^{1}\left(E_{K}\right) .
$$

If $K \hookrightarrow L$ is a trivial cofibration, then Condition (Q2) of Definition 2.8 ensures that $\alpha$ is surjective and $\beta$ an isomorphism, so the obstruction is zero and the lift exists, proving that $\underline{\operatorname{Hom}}_{\mathcal{G}}\left(\omega, \omega^{\prime}\right)$ is quasi-smooth, as required.

Now, the inclusions $\mathrm{MC}_{E_{0}} \hookrightarrow \mathrm{MC}(E)$ and $\underline{\operatorname{Hom}}_{\mathcal{G}}\left(\omega, \omega^{\prime}\right) \hookrightarrow E^{0}$ define a morphism

$$
\alpha: \bar{W} \mathcal{G} \rightarrow \pi^{0} \mathfrak{D} \mathfrak{e f}(E)
$$

of quasi-smooth functors $\mathcal{C}_{\Lambda} \rightarrow$ Set. Note that $\bar{W} \mathcal{G}_{0}=\mathrm{MC}_{E_{0}}=\pi^{0} \mathfrak{D} \mathfrak{e f}(E)_{0}$.

(Q2) also ensures that $\alpha$ is a weak equivalence on tangent spaces, with $\pi_{n} \bar{W} \mathcal{G}(k[\epsilon])=$ $\left.\mathrm{H}^{1-n}(\lrcorner E\right)$. As in the proof of Lemma 2.27, this implies that $\alpha(A)$ is a weak equivalence in $\mathbb{S}$ for all $A$.

Remark 3.15. If $\mathcal{B}$ has uniformly trivial deformation theory, then note that $\tilde{D}$ always exists, and that the pre-DDC $E$ of Theorem 3.13 automatically satisfies Definition 2.8.(Q1).

However, if $E$ just satisfies all the conditions of Theorem 3.13, then Propositions 2.23 and 2.25 then give a DDC $D E$, which by Lemma 2.27 also has $\bar{W} \mathcal{D}_{D \text {,id }} \sim \pi^{0} \mathfrak{D e f}(D E)$. 
3.3. Deformations of diagrams and invariance under weak equivalence. In a similar vein, we may study deformations of a morphism, or even of a diagram.

Definition 3.16. Define $\Delta_{* *}$ to be the subcategory of the ordinal number category $\Delta$ containing only those morphisms $f: \mathbf{m} \rightarrow \mathbf{n}$ with $f(0)=0, f(m)=n$. Given a category $\mathcal{C}$, a functor $X: \Delta_{* *} \rightarrow \mathcal{C}$ consists of objects $X^{n} \in \mathcal{C}$, with all of the operations $\partial^{i}, \sigma^{i}$ of a cosimplicial complex except $\partial^{0}, \partial^{n+1}: X^{n} \rightarrow X^{n+1}$.

Definition 3.17. Given a monoidal category $\mathcal{C}$ and a set $\mathcal{O}$, recall from [Pri3] that a $\mathcal{C}$-valued quasi-descent datum $X$ on objects $\mathcal{O}$ consists of:

(1) objects $X(a, b) \in \mathcal{C}^{\Delta_{* *}}$ for all $a, b \in \mathcal{O}$;

(2) morphisms $X(a, b)^{m} \otimes X(b, c)^{n} \stackrel{*}{\rightarrow} X(a, c)^{m+n}$ making the following diagram commute for all $, b, c \in \mathcal{O}$

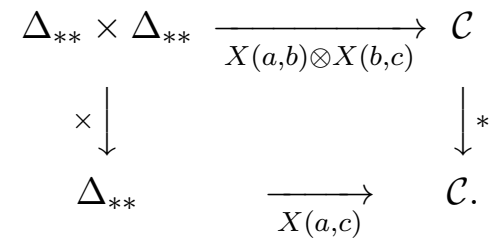

(3) morphisms $1 \rightarrow X(a, a)^{0}$ for all $a \in \mathcal{O}$, acting as the identity for the multiplication $*$.

Note that a pre-DDC over $\Lambda$ is a quasi-descent datum (on one object) in the monoidal category $(s \mathrm{Sp}, \times)$.

Definition 3.18. Let $Q \operatorname{Dat}(\mathcal{C})$ be the category of $\mathcal{C}$-valued quasi-descent data, i.e. of pairs $(\mathcal{O}, X)$ for $\mathcal{O}$ a set and $X$ a quasi-descent datum on objects $\mathcal{O}$.

We say that $\mathcal{D}$ is an enrichment of a $\mathcal{C}$-enriched category $\mathcal{F}$ if $\mathrm{Ob} \mathcal{F} \cong \mathrm{Ob} \mathcal{D}$ and $\mathcal{F}(x, y) \cong \mathcal{D}^{0}(x, y)$, compatible with the product and identities.

Proposition 3.19. For a diagram of simplicial category-valued functors as in §3.2, the $s \mathrm{Sp}$-enriched category $\mathcal{B}(\Lambda)$ has a natural enrichment in QDat $(s \mathrm{Sp})$. If the simplicial structure on $\mathcal{B}$ is constant, then this enrichment is in QDat(Sp).

Proof. This is just [Pri3] Proposition ??. The enriched Hom-set $\underline{\mathscr{H} o m}\left(B, B^{\prime}\right): \mathcal{C}_{\Lambda} \rightarrow \mathbb{S}^{\Delta_{* *}}$ is given by

$$
\underline{\mathscr{H} o m}^{n}\left(B, B^{\prime}\right):=\underline{\operatorname{Hom}}_{\mathcal{B}}\left(\top_{\mathrm{h}}^{n} B, \perp_{\mathrm{v}}^{n} B^{\prime}\right) .
$$

If the simplicial structure on $\mathcal{B}$ is constant, then $\underline{\operatorname{Hom}}_{\mathcal{B}}=\operatorname{Hom}_{\mathcal{B}}$, so $\underline{\mathscr{H} \text { om }}\left(B, B^{\prime}\right)$ lies in Sp.

Definition 3.20. Given a morphism $f: D \rightarrow D^{\prime}$ in $\mathcal{D}(k)$ for which $U V D, U V D^{\prime}$ lift to $B, B^{\prime}$ in $\mathcal{B}(\Lambda)$, define

$$
E_{\mathcal{D} / \mathcal{B}}^{n}(f):=\underline{\mathscr{H} o m}^{n}\left(B, B^{\prime}\right)_{U V\left(\alpha_{D^{\prime}}^{n} \circ f \circ \varepsilon_{D}^{n}\right)} \in s \mathrm{Sp}
$$

Write $E_{\mathcal{D} / \mathcal{B}}^{*}(D):=E_{\mathcal{D} / \mathcal{B}}^{*}\left(\operatorname{id}_{D}\right)$.

Definition 3.21. Given a morphism $f: D \rightarrow D^{\prime}$ in $\mathcal{D}(k)$ for which $E_{\mathcal{D} / \mathcal{B}}^{*}(f) \in\left(s \mathrm{Sp}_{k}\right)^{\Delta_{* *}}$ is levelwise smooth, define

$$
\mathrm{C}_{\mathcal{D} / \mathcal{B}}^{\bullet}(f):=\tan E_{\mathcal{D} / \mathcal{B}}^{*}(f),
$$

and note that that this becomes a cosimplicial complex (of simplicial complexes), by [Pri5] Lemma 3.10. Explicitly,

$$
\mathrm{C}_{\mathcal{D} / \mathcal{B}}^{n}(f)=\tan \underline{\operatorname{Hom}}_{\mathcal{D} \mid \mathcal{C}_{k}}\left(\perp_{\mathrm{h}}^{n+1} D, \top_{\mathrm{h}}^{n+1} D^{\prime}\right) \alpha_{D^{\prime}}^{n+1} \circ f \circ \varepsilon_{D}^{n} .
$$


Define

$$
\begin{aligned}
& \underline{\operatorname{Ext}}_{\mathcal{D} / \mathcal{B}}^{n}(f):=\mathrm{H}^{n}\left(\mathrm{C}_{\mathcal{D} / \mathcal{B}}^{\bullet}(f)\right) \in s \operatorname{Vect}_{k} \\
& \operatorname{Ext}_{\mathcal{D} / \mathcal{B}}^{n}(f):=\mathrm{H}^{n}\left(\operatorname{Tot} N \mathrm{C}_{\mathcal{D} / \mathcal{B}}^{\bullet}(f)\right) \in \operatorname{Vect}_{k} .
\end{aligned}
$$

Definition 3.22. Say that a morphism $f: D \rightarrow D^{\prime}$ in $\mathcal{D}(k)$ is Q2 over $\mathcal{B}$ if

(1) $U V D, U V D^{\prime}$ lift to $\mathcal{B}(\Lambda)$,

(2) $E_{\mathcal{D} / \mathcal{B}}^{*}(f)$ is levelwise smooth, and

(3) $\underline{\operatorname{Ext}}_{\mathcal{D} / \mathcal{B}}^{i}(f)$ is a constant simplicial complex for $i>0$.

We say that $f$ is quasi-smooth over $\mathcal{B}$ if in addition $\mathrm{H}_{*} \mathrm{C}_{\mathcal{D} / \mathcal{B}}^{n}(f)=0$ for all $n$.

Remark 3.23. Note that if $f$ is Q2 over $\mathcal{B}$, then we have $\left.\operatorname{Ext}_{\mathcal{D} / \mathcal{B}}^{*}(f)=\mathrm{H}^{*}(\lrcorner N \mathrm{C}_{\mathcal{D} / \mathcal{B}}^{\bullet}(f)\right)$, by Lemma 2.12.

Definition 3.24. Given a small category $\mathbb{I}$, and an $\mathbb{I}$-diagram $\mathbb{D}: \mathbb{I} \rightarrow \mathcal{D}(k)$ with objects $U V \mathbb{D}(i)$ lifting to $\mathcal{B}(\Lambda)$, define the pre-DDC $E_{\mathcal{D} / \mathcal{B}}^{\bullet}(\mathbb{D})$ by

$$
E_{\mathcal{D} / \mathcal{B}}^{n}(\mathbb{D})=\prod_{i_{0} \stackrel{f_{1}}{\longrightarrow} i_{1} \underset{\text { in } \mathbb{I}}{\stackrel{f_{2}}{\longrightarrow} \ldots f_{n}} i_{n}} E^{n}\left(\mathbb{D}\left(f_{n} \circ f_{n-1} \circ \ldots f_{0}\right)\right)=\prod_{x \in B \mathbb{I}_{n}} E^{n}\left(\mathbb{D}\left(\partial_{1}{ }^{n-1} x\right)\right),
$$

where $B \mathbb{I}$ is the nerve of $\mathbb{I}$ (so $B \mathbb{I}_{0}=\mathrm{Ob}(\mathbb{I}), B \mathbb{I}_{1}=\operatorname{Mor}(\mathbb{I})$ ), and $\partial_{1}^{-1}:=\sigma_{0}$. The operations are defined as in Definition 2.22.

Lemma 3.25. Given an $\mathbb{I}$-diagram $\mathbb{D}: \mathbb{I} \rightarrow \mathcal{D}(k)$ with all morphisms $\mathbb{D}(f)$ quasi-smooth (resp. Q2) over $\mathcal{B}$, the pre-DDC $E_{\mathcal{D} / \mathcal{B}}^{\bullet}(\mathbb{D})$ is quasi-smooth (resp. is levelwise smooth and satisfies Definition 2.8.(Q2)).

Proposition 3.26. Given an $\mathbb{I}$-diagram $\mathbb{D}: \mathbb{I} \rightarrow \mathcal{D}(k)$ with all morphisms $Q 2$, the classifying space $\bar{W}\left(\mathcal{D}^{\mathbb{I}}\right)_{\mathbb{D} \text {,id }}$ and $\pi^{0} \mathfrak{D} \mathfrak{e} \mathfrak{f}\left(E_{\mathcal{D} / \mathcal{B}}^{\bullet}(\mathbb{D})\right)$ are canonically weakly equivalent as functors from $\mathcal{C}_{\Lambda}$ to $\mathbb{S}$.

Proof. This is just [Pri3] Lemma ??.

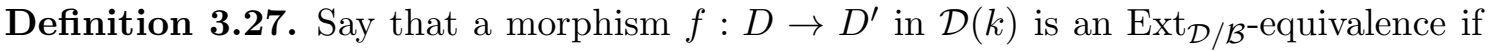
$U V D, U V D^{\prime}$ lift to $B, B^{\prime}$ in $\mathcal{B}(\Lambda)$, with $E^{*}(f)$ levelwise smooth, and the maps

$$
\operatorname{Ext}_{\mathcal{D} / \mathcal{B}}^{*}\left(\operatorname{id}_{D}\right) \stackrel{f_{*}}{\longrightarrow} \operatorname{Ext}_{\mathcal{D} / \mathcal{B}}^{*}(f) \stackrel{f^{*}}{\longleftarrow} \operatorname{Ext}_{\mathcal{D} / \mathcal{B}}^{*}\left(\operatorname{id}_{D^{\prime}}\right)
$$

are isomorphisms.

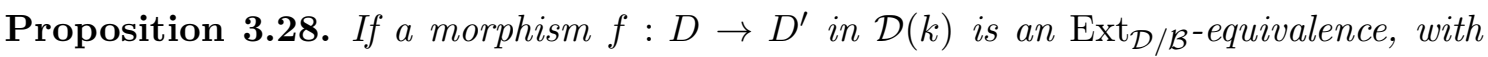
the morphisms $f, \operatorname{id}_{D}, \operatorname{id}_{D^{\prime}}$ all Q2, then the $D D C s D E_{\mathcal{D} / \mathcal{B}}^{\bullet}(D)$ and $D E_{\mathcal{D} / \mathcal{B}}^{\bullet}\left(D^{\prime}\right)$ are quasiisomorphic.

Proof. Let $\mathbb{I}:=(\bullet \rightarrow \bullet)$ be the category with two objects and one non-identity morphism, and consider the diagram $\mathbb{D}: \mathbb{I} \rightarrow \mathcal{D}(k)$ given by $D \stackrel{f}{\rightarrow} D^{\prime}$. By Lemma 3.25 and Proposition 2.23 , we know that the pre-DDCs $D E_{\mathcal{D} / \mathcal{B}}^{\bullet}(D), D E_{\mathcal{D} / \mathcal{B}}^{\bullet}\left(D^{\prime}\right)$ and $D E_{\mathcal{D} / \mathcal{B}}^{\bullet}(\mathbb{D})$ are all DDCs.

The inclusions of objects into II give morphisms $E_{\mathcal{D} / \mathcal{B}}^{\bullet}(D) \leftarrow E_{\mathcal{D} / \mathcal{B}}^{\bullet}(\mathbb{D}) \rightarrow E_{\mathcal{D} / \mathcal{B}}^{\bullet}\left(D^{\prime}\right)$. We just need to describe the cohomology groups $\left.\mathrm{H}^{*}(\lrcorner E_{\mathcal{D} / \mathcal{B}}^{\bullet}(\mathbb{D})\right)$ to show that these induce quasi-isomorphisms.

The tangent space $\mathrm{C}_{\mathcal{D} / \mathcal{B}}(\mathbb{D})$ is the diagonal cosimplicial complex associated to the bicosimplicial complex

$$
\prod_{x \in B \mathbb{I}_{m}} \mathrm{C}_{\mathcal{D} / \mathcal{B}}^{n}\left(\mathbb{D}\left(\partial_{1}{ }^{m-1} x\right)\right)
$$


whose horizontal normalisation is the cochain complex

$$
\mathrm{C}_{\mathcal{D} / \mathcal{B}}^{\bullet}\left(\mathrm{id}_{D}\right) \times \mathrm{C}_{\mathcal{D} / \mathcal{B}}^{\bullet}\left(\mathrm{id}_{D^{\prime}}\right) \stackrel{\left(f_{*},-f^{*}\right)}{\longrightarrow} \mathrm{C}_{\mathcal{D} / \mathcal{B}}^{\bullet}(f)
$$

in degrees 0 and 1.

Thus $\mathrm{C}_{\mathcal{D} / \mathcal{B}}^{\bullet}(\mathbb{D})$ is the mapping cone of the morphism $\left(f_{*},-f^{*}\right)$. Since $f$ is an $\operatorname{Ext}_{\mathcal{D} / \mathcal{B}^{-}}$ equivalence, we deduce that the maps $\left.\operatorname{Ext}_{\mathcal{D} / \mathcal{B}}^{*}\left(\mathrm{id}_{D}\right) \leftarrow \mathrm{H}^{*}(\lrcorner E_{\mathcal{D} / \mathcal{B}}^{\bullet}(\mathbb{D})\right) \rightarrow \operatorname{Ext}_{\mathcal{D} / \mathcal{B}}^{*}\left(\mathrm{id}_{D^{\prime}}\right)$ are indeed isomorphisms.

3.3.1. Constrained deformations. We now consider a generalisation, by taking a small diagram

$$
\mathbb{D}: \mathbb{I} \rightarrow \mathcal{D}(k),
$$

a subcategory $\mathbb{J} \subset \mathbb{I}$, and $\widetilde{\left.\mathbb{D}\right|_{\mathbb{J}}}: \mathbb{J} \rightarrow \mathcal{D}(\Lambda)$ lifting $\left.\mathbb{D}\right|_{\mathbb{J}}$. We wish to describe deformations of $\mathbb{D}$ which agree with $\left.\widetilde{\mathbb{D}}\right|_{\mathbb{J}}$ on $\mathbb{J}$. Note that when $\mathbb{I}=(0 \rightarrow 1)$ and $\mathbb{J}=\{1\}$, this is the type of problem considered in [FM] and [Ran].

Proposition 3.29. Given an $\mathbb{I}$-diagram $\mathbb{D}: \mathbb{I} \rightarrow \mathcal{D}(k)$ with all morphisms Q2, and with $\left.\widetilde{\mathbb{D}}\right|_{\mathbb{J}}$ as above, the simplicial groupoid of deformations of $\mathbb{D}$ fixing $\left.\widetilde{\mathbb{D}}\right|_{\mathbb{J}}$ is governed by the pre- $D D C$

$$
E_{\mathcal{D} / \mathcal{B}}^{\bullet}(\mathbb{D}) \times_{E_{\mathcal{D} / \mathcal{B}}^{\bullet}(\mathbb{D} \mid J)} \bullet
$$

where $\bullet \rightarrow E_{\mathcal{D} / \mathcal{B}}^{\bullet}\left(\left.\mathbb{D}\right|_{\mathbb{J}}\right)$ is defined by the object of $\mathrm{MC}\left(E_{\mathcal{D} / \mathcal{B}}^{\bullet}\left(\left.\mathbb{D}\right|_{\mathbb{J}}\right)\right)_{0}$ corresponding to $\left.\widetilde{\mathbb{D}}\right|_{\mathbb{J}}$.

Proof. We need to show that the classifying space

$$
\bar{W}\left(\mathcal{D}^{\mathbb{I}} \times\left._{\mathcal{D}^{\mathbb{J}}}^{h} \widetilde{\mathbb{D}}\right|_{\mathbb{J}}\right) \mathbb{D}, \text { id }
$$

of the homotopy fibre of simplicial categories is canonically weakly equivalent to

as a functor from $\mathcal{C}_{\Lambda}$ to $\mathbb{S}$.

$$
\pi^{0} \mathfrak{D} \mathfrak{e f}\left(E_{\mathcal{D} / \mathcal{B}}^{\bullet}(\mathbb{D}) \times_{E_{\mathcal{D} / \mathcal{B}}^{\bullet}}\left(\left.\mathbb{D}\right|_{\mathbb{J}}\right) \bullet\right)
$$

We know that the functor $\mathfrak{D e f}$ preserves inverse limits, so

$$
\pi^{0} \mathfrak{D} \mathfrak{e f}\left(E^{\bullet}(\mathbb{D}) \times_{E \bullet\left(\left.\mathbb{D}\right|_{\mathbb{J}}\right)} \bullet\right)=\pi^{0} \mathfrak{D} \mathfrak{e f}\left(E_{\mathcal{D} / \mathcal{B}}^{\bullet}(\mathbb{D})\right) \times_{\pi^{0} \mathfrak{D} \mathfrak{f}\left(E^{\bullet}\left(\left.\mathbb{D}\right|_{\mathbb{J}}\right)\right)} \bullet
$$

By Lemma 2.27, Lemma 3.25 and Corollary 2.21, we know that $\pi^{0} \mathfrak{D} \mathfrak{e f}\left(E_{\mathcal{D} / \mathcal{B}}^{\bullet}(\mathbb{D})\right)(A) \rightarrow$ $\pi^{0} \mathfrak{D} \mathfrak{e f}\left(E^{\bullet}(\mathbb{D} \mid \mathbb{J})\right)(A)$ is a fibration in $\mathbb{S}$, so the fibre over any point is the homotopy fibre. Proposition 3.26 now shows that this is equivalent to the homotopy fibre of $\bar{W}\left(\mathcal{D}(A)^{\mathbb{I}}\right) \rightarrow$ $\bar{W}\left(\mathcal{D}(A)^{\mathbb{J}}\right)$ over $\left.\widetilde{\mathbb{D}}\right|_{\mathbb{J}}$, as required.

\section{EXAMPLES}

We now show how to apply Theorem 3.13, combining it with Definitions 2.16 or 2.19 to obtain derived deformation functors. This gives many new examples coming from categories with non-trivial simplicial structure.

4.1. Chain complexes. We will denote chain complexes by $V_{\bullet}$, and their underlying graded modules by $V_{*}$.

Definition 4.1. Define $d g \mathrm{FMod}(A)$ to be the category of chain complexes of flat modules over $A$. We make this into a simplicial category by defining the simplicial normalisation $N^{s} \underline{\operatorname{Hom}}\left(U_{\bullet}, V_{\bullet}\right)$ to be the chain complex

$$
N_{n}^{s} \underline{\operatorname{Hom}}\left(U_{\bullet}, V_{\bullet}\right):=\left\{\begin{array}{cc}
\operatorname{Hom}\left(U_{\bullet}, V_{\bullet}\right) & n=0 \\
\prod_{i \geq 0} \operatorname{Hom}\left(U_{i}, V_{i+n}\right) & n>0
\end{array},\right.
$$

with boundary $\operatorname{map} \operatorname{ad}_{d}(f):=d \circ f \pm f \circ d$. This determines the simplicial module $\underline{\operatorname{Hom}}\left(U_{\bullet}, V_{\bullet}\right):=\left(N^{s}\right)^{-1} N^{s} \underline{\operatorname{Hom}}\left(U_{\bullet}, V_{\bullet}\right)$ by the Dold-Kan correspondence. 
Definition 4.2. Define $g \mathrm{FMod}(A)$ to be the category of flat $\mathbb{N}_{0}$-graded modules over $A$, with the simplicial structure

$$
N_{n}^{s} \underline{\operatorname{Hom}}\left(U_{*}, V_{*}\right):=\left\{\begin{array}{cc}
\operatorname{Hom}\left(U_{*}, V_{*}\right) & n=0 \\
\operatorname{Hom}\left(U_{*}, V_{*}[n]\right) \times \operatorname{Hom}\left(U_{*}, V_{*}[n-1]\right) & n>0
\end{array},\right.
$$

where the boundary map is given by $d(f, g):=(g, 0)$.

Lemma 4.3. The functor $g \mathrm{FMod}: \mathcal{C}_{\Lambda} \rightarrow s$ Cat has uniformly trivial deformation theory.

Proof. Since flat $A$-modules are free, it follows that objects lift. The other properties from Definition 3.12 now follow by a simple calculation.

Definition 4.4. Let the forgetful functor $d g$ FMod $\rightarrow g$ FMod be given by $V_{\bullet} \mapsto V_{*}$, and defined on simplicial morphisms by mapping $f \in N_{n}^{s} \underline{\operatorname{Hom}}\left(U_{\bullet}, V_{\bullet}\right)$ to $\left(f, \operatorname{ad}_{d}(f)\right) \in$ $N_{n}^{s} \underline{\operatorname{Hom}}\left(U_{*}, V_{*}\right)$.

Lemma 4.5. The forgetful functor $d g \mathrm{FMod} \rightarrow g \mathrm{FMod}$ of simplicial categories has a right adjoint $G$, and the resulting adjunction is comonadic.

Proof. Define $\left(G V_{*}\right)_{n}:=V_{n} \oplus V_{n-1}$, with $d(v, w)=(w, 0)$. The unit $\alpha: U_{\bullet} \rightarrow G\left(U_{*}\right)$ of the adjunction is $\alpha(u)=(u, d u)$, for any chain complex $U_{\bullet}$, and the co-unit $\gamma: G\left(V_{*}\right) \rightarrow V_{*}$ is the map $\gamma(v, w)=v$ of graded modules.

Let $\perp=V G$ and $\top=G V$, for $V$ the forgetful functor.

Proposition 4.6. For $U_{\bullet} \in d g \mathrm{FMod}(k)$, the pre- $D D C$

$$
E^{n}(A):=\underline{\operatorname{Hom}}_{g \operatorname{FMod}_{A}}\left(\tilde{U}_{*} \otimes A, \perp^{n} \tilde{U}_{*} \otimes A\right)_{V\left(\alpha_{U}^{n}\right)}
$$

of Theorem 3.13 is quasi-smooth, with cohomology

$$
\begin{aligned}
& \left.\mathrm{H}^{*}(\lrcorner E\right)=\mathrm{H}^{*}\left(\ldots \stackrel{\operatorname{ad}_{d}}{\longrightarrow} \operatorname{Hom}_{g_{\mathbb{Z}} \operatorname{Mod}_{k}}\left(U_{*}, U_{*}[-n]\right) \stackrel{\mathrm{ad}_{d}}{\longrightarrow} \operatorname{Hom}_{g_{\mathbb{Z}} \operatorname{Mod}_{k}}\left(U_{*}, U_{*}[-n-1]\right) \stackrel{\operatorname{ad}_{d}}{\longrightarrow} \ldots\right) \\
& =\mathbb{E x t}_{d g_{\mathbb{Z}} \operatorname{Vect}_{k}}^{*}\left(U_{\bullet}, U_{\bullet}\right) \text {, }
\end{aligned}
$$

for $d g_{\mathbb{Z}}$ Vect $_{k}$ the category of $\mathbb{Z}$-graded chain complexes over $k$, and $\mathbb{E} x t$ the hyperext functor of [Wei] $§ 10.7$.

Proof. Observe that $\mathrm{H}^{*}\left(E_{n}\right)$ is cohomology of the complex

$$
\underline{\operatorname{Hom}}_{n}\left(U_{\bullet}, \top U_{\bullet}\right) \rightarrow \underline{\operatorname{Hom}}_{n}\left(U_{\bullet}, \top^{2} U_{\bullet}\right) \rightarrow \ldots
$$

associated to the monad $\top$ (as in [Wei] $\S 8.7$ ), so for $n>0, N_{n}^{s} \mathrm{H}^{*}(E)=\mathrm{H}^{*}\left(N_{n}^{s} E\right.$ ) is cohomology of the complex

$$
\operatorname{Hom}\left(U_{*},(\top U)_{*}[n]\right) \rightarrow \operatorname{Hom}\left(U_{*},\left(\top^{2} U\right)_{*}[n]\right) \rightarrow \ldots
$$

Now, $(\top U)_{*}=\perp\left(U_{*}\right)$, and the augmented cosimplicial complex $U_{*} \longrightarrow \perp\left(U_{*}\right) \Longrightarrow \perp^{2}\left(U_{*}\right) \longrightarrow \ldots$ is canonically contractible (in the sense of [Wei] 8.4.6), giving

$$
N_{n}^{s} \mathrm{H}^{i}(E)=\left\{\begin{array}{cc}
\operatorname{Hom}\left(U_{*}, U_{*}[n]\right) & i=0 \\
0 & i>0,
\end{array}\right.
$$

for $n>0$, so $E$ is quasi-smooth, and $\left.\mathrm{H}^{-n}(\lrcorner E\right)=\mathbb{E x t}_{d g_{\mathbb{Z}} \operatorname{Vect}_{k}}^{-n}\left(U_{\bullet}, U_{\bullet}\right)$ for $n \geq 0$.

Since $\operatorname{ker}\left(\gamma_{U}: \perp U_{*} \rightarrow U_{*}\right)=U_{*}[-1]$, the cosimplicial normalisation $N_{c}^{n}\left(\perp^{\bullet} U_{*}\right)=$ $N_{c}^{n-1}\left(\perp^{\bullet} U_{*}\right)[-1]$, so $N_{c}^{n}\left(\perp^{\bullet} U_{*}\right)=U_{*}[-n]$. Thus $N_{c} \mathrm{C}^{\bullet}\left(E_{0}\right)$ is just

$$
\left.\left.\operatorname{Hom}\left(U_{*}, U_{*}\right) \stackrel{\operatorname{ad}_{d}}{\longrightarrow} \operatorname{Hom}\left(U_{*}, U_{*}[-1]\right)\right) \stackrel{\operatorname{ad}_{d}}{\longrightarrow} \operatorname{Hom}\left(U_{*}, U_{*}[-2]\right)\right) \stackrel{\operatorname{ad}_{d}}{\longrightarrow} \ldots,
$$

so $\left.\mathrm{H}^{i}(\lrcorner E\right)=\mathbb{E x t}_{d g_{\mathbb{Z}} \operatorname{Vect}_{k}}^{i}\left(U_{\bullet}, U_{\bullet}\right)$ for $i>0$.

Remarks 4.7. (1) Dually, we may consider deformations of (non-negatively graded) cochain complexes. This simplicial category is monadic over graded modules. 
(2) We may incorporate the constructions of this section into more interesting examples. For instance, deformations of a complex of $\mathscr{O}_{X}$-modules on an algebraic space $X$ are given by considering the diagram

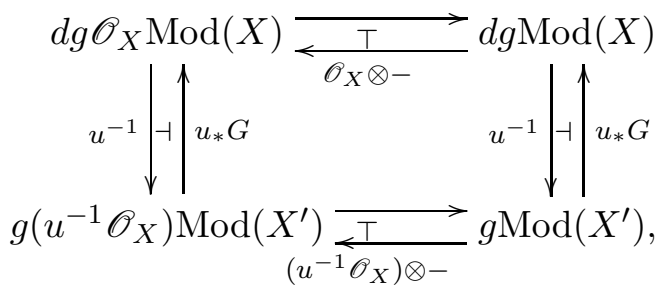

of simplicial categories, where $u: X^{\prime} \rightarrow X$ is the map to $X$ from its set of geometric points. The resulting pre-DDC will be quasi-smooth whenever $\operatorname{Ext}_{\mathscr{O}_{X}}^{i}\left(M_{m}, M_{n}\right)=$ 0 for all $i>0$ and $n>m$.

\subsection{Simplicial complexes.}

Definition 4.8. Define $s \mathrm{FMod}(A)$ to be the category of simplicial flat modules over $A$. We make this into a simplicial category by setting

$$
\underline{\operatorname{Hom}}\left(U_{\bullet}, V_{\bullet}\right)_{n}:=\operatorname{Hom}\left(\Delta^{n} \otimes U_{\bullet}, V_{\bullet}\right),
$$

where, for a set $X$ and module $U$, we set $U \otimes X:=\bigoplus_{x \in X} U$.

Definition 4.9. Define $\Delta_{*}$ to be the subcategory of the ordinal number category $\Delta$ containing only those morphisms fixing 0 . Given a category $\mathcal{C}$, define the category $s_{+} \mathcal{C}$ of almost simplicial complexes (resp.the category $c_{+} \mathcal{C}$ of almost cosimplicial complexes) in $\mathcal{C}$ to consist of functors $\left(\Delta_{*}\right)$ opp $\rightarrow \mathcal{C}$ (resp. $\Delta_{*} \rightarrow \mathcal{C}$ ). Thus an almost simplicial object $X_{*}$ consists of objects $X_{n} \in \mathcal{C}$, with all of the operations $\partial_{i}, \sigma_{i}$ of a simplicial complex except $\partial_{0}$, satisfying the usual relations. Similarly, an almost cosimplicial complex has all of the coface and coboundary operations except $\partial^{0}$.

From now on, we will denote simplicial sets by $X_{\bullet}$, and their underlying almost simplicial complexes by $X_{*}$.

Definition 4.10. Define a simplicial structure on the category $s_{+} \mathrm{FMod}(A)$ by setting

$$
\underline{\operatorname{Hom}}\left(U_{*}, V_{*}\right)_{n}:=\operatorname{Hom}\left(\Delta_{*}^{n} \otimes U_{*}, V_{*}\right) .
$$

Remark 4.11. Recall that the Dold-Kan correspondence gives an equivalence $N: s \operatorname{Mod} \rightarrow$ $d g$ Mod of categories, by the formula $N(V)_{n}=\bigcap_{i=1}^{n} \operatorname{ker}\left(\partial_{i}: V_{n} \rightarrow V_{n-1}\right)$, with $d:=\partial_{0}$. Observe that this extends to an equivalence $N: s_{+} \operatorname{Mod} \rightarrow g \operatorname{Mod}$ of categories, given by the same formula. This is only a weak equivalence of simplicial categories, not an equivalence.

Lemma 4.12. The forgetful functor $s$ FMod $\rightarrow s_{+}$FMod of simplicial categories has a right adjoint $G_{\partial}$, and the resulting adjunction is comonadic.

Proof. Let $\left(G_{\partial} V_{*}\right)_{n}:=V_{n} \oplus V_{n-1} \oplus \ldots \oplus V_{0}$, with operations

$$
\begin{array}{r}
\partial_{i}\left(v_{n}, \ldots, v_{0}\right)=\left(\partial_{i} v_{n}, \partial_{i-1} v_{n-1}, \ldots, \partial_{1} v_{n-i+1}, v_{n-i-1}, \ldots, v_{1}, v_{0}\right) \\
\sigma_{i}\left(v_{n}, \ldots, v_{0}\right)=\left(\sigma_{i} v_{n}, \sigma_{i-1} v_{n-1}, \ldots, \sigma_{0} v_{n-i}, v_{n-i}, \ldots, v_{1}, v_{0}\right) .
\end{array}
$$

The unit $\alpha: U_{\bullet} \rightarrow\left(G_{\partial} U_{*}\right)_{\bullet}$ of the adjunction is

$$
\alpha(u)=\left(u, \partial_{0} u, \partial_{0}{ }^{2} u, \ldots, \partial_{0}{ }^{n} u\right),
$$

for any simplicial complex $U_{\bullet}$, and $u \in U_{n}$. The co-unit $\gamma: G_{\partial} V_{*} \rightarrow V_{*}$ is the map $\gamma\left(v_{n}, \ldots, v_{0}\right)=v_{n}$ of almost simplicial complexes. 
Remark 4.13. The forgetful functor $V_{\partial}$ also has a left adjoint, which does not respect the simplicial structure of the categories. It is given by $\mathcal{L}_{\partial}\left(V_{*}\right)_{n}=V_{n+1}$, with $\partial_{i}^{\mathcal{L}_{\partial} V}=\partial_{i+1}^{V}$, $\sigma_{i}^{\mathcal{L}_{\partial} V}=\sigma_{i+1}^{V}$, and unit $\sigma_{0}: V_{*} \rightarrow V_{\partial} \mathcal{L}_{\partial}\left(V_{*}\right)=\mathcal{L}_{\partial}(V)_{*}$. Note that $\mathcal{L}_{\partial} V_{\partial}$ is the functor $\mathrm{DEC}^{\text {opp }}$ defined on simplicial sets in [Gle].

Definition 4.14. Define objects $\Xi^{n} \in s_{+}$Set by $\Xi_{m}^{n}=\operatorname{Hom}_{\Delta_{*}}([m],[n])$, and let $\partial \Xi^{n}$ be the boundary of $\Xi^{n}$ (i.e. the union of the images of all maps $\Xi^{n-1} \rightarrow \Xi^{n}$ ). Note that $\mathcal{L}\left(\Xi^{n}\right)=\Delta^{n}$ and, for $n>0, \mathcal{L}\left(\partial \Xi^{n}\right)=\Lambda_{0}^{n}$, the 0th horn.

Lemma 4.15. For $X \in s_{+}$Set, $X_{0} \rightarrow \mathcal{L}(X)$ is a weak equivalence.

Proof. Any injective map $f: Z \rightarrow X$ in $s_{+}$Set is an inductive limit of pushouts of maps $\partial \Xi^{n} \rightarrow \Xi^{n}$ for $n \geq 0$. If $f_{0}: Z_{0} \rightarrow X_{0}$ is an isomorphism, we may take $n>0$ only. Then $\mathcal{L}(f): \mathcal{L}(Z) \rightarrow \mathcal{L}(X)$ is an inductive limit of pushouts of maps $\Lambda_{0}^{n} \rightarrow \Delta^{n}$, so is a trivial cofibration. Taking $Z=X_{0}$ gives the required result.

Let $\perp=V_{\partial} G_{\partial}$ and $\top=G_{\partial} V_{\partial}$, for $V_{\partial}: s \mathrm{FMod}(A) \rightarrow s_{+} \mathrm{FMod}(A)$ the forgetful functor.

Proposition 4.16. $s_{*}$ FMod has uniformly trivial deformation theory, so given $U_{\bullet} \in$ $s \operatorname{FMod}(k)$, we may lift $U_{*} \in s_{+} \operatorname{FMod}(k)$ to $\tilde{U}_{*} \in s_{+} \operatorname{FMod}(\Lambda)$. The conditions of Theorem 3.13 are satisfied, and the pre-DDC

$$
E^{n}(A):=\underline{\operatorname{Hom}}_{s_{+}} \operatorname{FMod}(A)\left(\tilde{U}_{*}, \perp^{n} \tilde{U}_{*}\right)_{V\left(\alpha_{U}^{n}\right)}
$$

is then a DDC, with cohomology $\left.\mathrm{H}^{*}(\lrcorner E\right)$ given by the complex

$$
\ldots \stackrel{\mathrm{ad}_{d}}{\longrightarrow} \operatorname{Hom}_{g_{\mathbb{Z}} \operatorname{Mod}_{k}}\left(N U_{*}, N U_{*}[-n]\right) \stackrel{\mathrm{ad}_{d}}{\longrightarrow} \operatorname{Hom}_{g_{\mathbb{Z}} \operatorname{Mod}_{k}}\left(N U_{*}, N U_{*}[-n-1]\right) \stackrel{\mathrm{ad}_{d}}{\longrightarrow} \ldots,
$$

i.e. $\mathbb{E x t}_{d g_{\mathbb{Z}} \operatorname{Vect}_{k}}^{*}\left(N U_{\bullet}, N U_{\bullet}\right)$.

Proof. This is similar to Proposition 4.6. The only difficulty lies in establishing Definition 2.8.(Q2'):

$$
N_{n} \mathrm{C}^{i}(E)=N_{n} \underline{\operatorname{Hom}}_{s \mathrm{FMod}(k)}\left(U_{\bullet}, \top^{i+1} U_{\bullet}\right)=\operatorname{Hom}_{s \operatorname{FMod}(k)}\left(U_{\bullet} \otimes\left(\Delta^{n} / \Lambda_{0}^{n}\right), \top^{i+1} U_{\bullet}\right) .
$$

Now, since $k \otimes \Lambda_{0}^{n} \rightarrow k \otimes \Delta^{n}$ is a weak equivalence admitting a retraction, $P:=$ $U_{\bullet} \otimes\left(\Delta^{n} / \Lambda_{0}^{n}\right)$ is trivially cofibrant, so is a projective object in the category $s \mathrm{FMod}(k)$. The cosimplicial complex $U^{\bullet}$ in $s \mathrm{FMod}(k)$ given by $U^{i}:=\top^{i+1} U_{\bullet}$ is a resolution of $U:=U_{\bullet}$ (since the augmented cosimplicial complex $V_{\partial} U \rightarrow V_{\partial} U^{\bullet}$ is contractible). Thus

$$
\mathrm{H}^{*} N_{n} \mathrm{C}^{\bullet}(E)=\mathbb{E x t}_{s \mathrm{FMod}(k)}^{*}\left(P, U^{\bullet}\right)=\operatorname{Ext}_{s \mathrm{FMod}(k)}^{*}(P, U)=\operatorname{Hom}_{s \mathrm{FMod}(k)}(P, U) .
$$

We have therefore shown that for $n>0$,

$$
N_{n} \mathrm{H}^{i}(E)=\left\{\begin{array}{cc}
N_{n} \underline{\operatorname{Hom}}_{s \operatorname{Fod}(k)}\left(U_{\bullet}, U_{\bullet}\right) & i=0 \\
0 & i>0 .
\end{array}\right.
$$

4.3. Simplicial algebras. Although the results in this section are expressed for commutative algebras, they will hold for any category equipped with a suitable forgetful functor to flat modules, and in particular algebras over any operad.

Definition 4.17. Let $\mathrm{FAlg}(A)$ be the category of flat (commutative) $A$-algebras, with $s \mathrm{FAlg}(A):=\mathrm{FAlg}(A)^{\Delta^{\mathrm{opp}}}$ and $s_{+} \mathrm{FAlg}(A):=\mathrm{FAlg}(A)^{\Delta_{*}^{\text {opp }}}$. Recall that, for $K \in \mathbb{S}$ and $R \in s \mathrm{FAlg}(A)$, we define $R \otimes K \in s \mathrm{FAlg}(A)$ by

$$
(R \otimes K)_{n}:=R_{n}^{\otimes K_{n}}=\overbrace{R_{n} \otimes R_{n} \otimes \ldots \otimes R_{n}}^{\left|K_{n}\right|} .
$$

Define $\otimes K: s_{+}$FAlg $\rightarrow s_{+}$FAlg by the same formula. 
Now, we make $s \mathrm{FAlg}(A), s_{+} \mathrm{FAlg}(A)$ into simplicial categories by setting

$$
\begin{aligned}
\underline{\operatorname{Hom}}_{s \mathrm{FAlg}}\left(R_{\bullet}, S_{\bullet}\right)_{n} & :=\operatorname{Hom}_{s \mathrm{FAlg}}\left(\Delta^{n} \otimes R_{\bullet}, S_{\bullet}\right), \\
\underline{\operatorname{Hom}}_{s_{+}} \mathrm{FAlg} & \left(R_{*}, S_{*}\right)_{n}:=\operatorname{Hom}_{s_{+}} \mathrm{FAlg}\left(\Delta_{*}^{n} \otimes R_{*}, S_{*}\right) .
\end{aligned}
$$

We now consider the commutative diagram

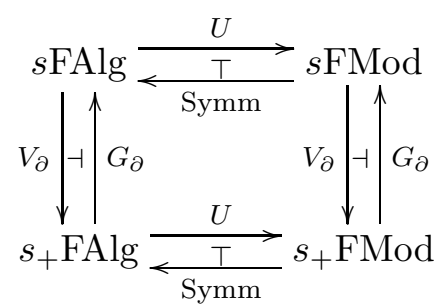

of adjunctions of homogeneous simplicial category-valued functors on $\mathcal{C}_{\Lambda}$.

Definition 4.18. Given an almost simplicial $A$-algebra $R_{*}$, define the category $s_{+} \operatorname{Mod}\left(R_{*}\right)$ to consist of almost simplicial $A$-modules $M_{*}$ equipped with an associative multiplication $R_{*} \otimes M_{*} \rightarrow M_{*}$, respecting the almost simplicial structures. This has a simplicial model structure (by applying [Hir] Theorem 11.3.2 to the forgetful functor $\left.s_{+} \operatorname{Mod}\left(R_{*}\right) \rightarrow s_{+} \operatorname{Mod}(A)\right)$.

All objects of $s_{+} \operatorname{Mod}\left(R_{*}\right)$ are fibrant. Since $A \otimes\left(\partial \Delta^{n}\right)_{*} \rightarrow A \otimes\left(\Delta^{n}\right)_{*}$ has a retraction in $s_{+} \operatorname{Mod}(A)$, we see that for all cofibrant $C_{*} \in s_{+} \operatorname{Mod}\left(R_{*}\right)$,

$$
\underline{\operatorname{Hom}}_{s_{+} \operatorname{Mod}\left(R_{*}\right)}\left(C_{*}, M_{*}\right)
$$

is trivially fibrant.

Proposition 4.19. Fix $R_{\bullet} \in s \mathrm{FAlg}(k)$, set $M_{*}:=U V_{\partial} R_{\bullet}$, and choose a lift $\tilde{M}_{*} \in$ $s_{+} \operatorname{FMod}(\Lambda)$.

Theorem 3.13 gives a pre- $D D C$

$$
E^{n}:=\underline{\operatorname{Hom}}_{s_{+} \text {FMod }}\left((U \operatorname{Symm})^{n} \tilde{M}_{*},\left(V_{\partial} G_{\partial}\right)^{n} \tilde{R}_{*}\right)_{U V_{\partial}\left(\alpha_{R}^{n} \circ \varepsilon_{R}^{n}\right)} .
$$

Moreover, if $R_{\bullet} \in s \mathrm{FAlg}(k)$ is cofibrant, then the pre-DDC E of Theorem 3.13 is a $D D C$. The almost simplicial k-algebra $R_{*}$ then lifts to $\tilde{R}_{*} \in s_{+} \mathrm{FAlg}(\Lambda)$, and $E$ is quasiisomorphic to the DDC defined by

$$
\left(E^{\prime}\right)_{n}=\underline{\operatorname{Hom}}_{s_{+}} \operatorname{FAlg}\left(\tilde{R}_{*},\left(V_{\partial} G_{\partial}\right)^{n} \tilde{R}_{*}\right)_{V_{\partial}\left(\alpha_{R}^{n}\right)}
$$

coming from the comonadic adjunction sFAlg $\underset{G_{\partial}}{\stackrel{V_{\partial}}{\longrightarrow}} s_{+}$FAlg.

Proof. It is straightforward to verify [Pri2] equations 1-4, since all our constructions commute with forgetful functors, so $E$ is a pre-DDC. Since $s_{+}$FMod is uniformly of trivial deformation theory, Definition 2.8.(Q1) is satisfied by $E$.

To establish quasi-smoothness, we must compute cohomology groups. Given a $k$-algebra $S$, recall that the cotangent complex is given by $\mathbb{L}_{n}(S / k)=J_{n} /\left(J_{n}\right)^{2}$, where $J_{n}$ is the kernel of the diagonal map $(\operatorname{Symm} U)^{n+1}(S) \otimes_{k} S \rightarrow S$. The cosimplicial complex $\mathrm{C}^{\bullet}\left(E_{n}\right)$ is then given by $\mathrm{C}^{m}\left(E_{n}\right)=\operatorname{Hom}_{s_{+} \operatorname{Mod}\left(R_{*}\right)}\left(\mathbb{L}_{m}(R / k)_{*} \otimes \Delta^{n}, G_{\partial}^{m} R_{*}\right)$. Thus $\mathrm{H}^{*}\left(E_{n}\right)$ is the total cohomology of the double complex

$$
\mathrm{C}^{i j}=\operatorname{Hom}_{s_{+} \operatorname{Mod}\left(R_{*}\right)}\left(\mathbb{L}_{i}(R / k)_{*} \otimes \Delta^{n}, G_{\partial}^{j} R_{*}\right)=\operatorname{Hom}_{s_{+} \operatorname{Mod}\left(R_{*}\right)}\left(\mathbb{L}_{i}(R / k)_{*},\left(G_{\partial}^{j} R_{*}\right)^{\Delta^{n}}\right) .
$$

Now, if $R_{\bullet}$ is cofibrant, the augmented complex $\mathbb{L}_{\bullet}\left(R_{*}\right) \rightarrow \Omega\left(R_{*} / k\right)$ is a levelwise cofibrant resolution in $s_{+} \operatorname{Mod}\left(R_{*}\right)$. Since all maps in $s_{+} \operatorname{Mod}\left(R_{*}\right)$ are weak equivalences, cofibrant modules are projective, so the complex is contractible.

Define André-Quillen cohomology on $s_{+} \operatorname{Mod}\left(R_{*}\right)$ by $D^{q}\left(R_{*} / k, M_{*}\right) \quad:=$ $\mathrm{H}^{q} \operatorname{Hom}_{s_{+} \operatorname{Mod}\left(R_{*}\right)}\left(\mathbb{L}_{\bullet}(R / k)_{*}, M_{*}\right)$. Given a small extension $A \rightarrow B$ with kernel $I$, 
and a flat almost simplicial $B$-algebra $S_{*}$, note that the obstruction to lifting $S_{*}$ to a flat $A$-algebra lies in $D^{2}\left(S_{*} / B, S_{*} \otimes_{B} I\right)=D^{2}\left(\left(S_{*} \otimes_{B} k\right) / k, S_{*} \otimes_{B} I\right)$, applying [Pri2] Theorem 2.2 to the adjunction

$$
s_{+} \mathrm{FAlg} \underset{\mathrm{Symm}}{\underset{T}{\longrightarrow}} s_{+} \mathrm{FMod} .
$$

This ensures that $R_{*}$ lifts to some $\tilde{R}_{*} \in s_{+} \operatorname{FAlg}(\Lambda)$, so $E^{\prime}$ can be defined.

Similarly to [Pri2] $\S 3.2 .2$, we see that $E^{\prime}$ is a levelwise smooth DDC, and that $\mathrm{H}^{*}\left(E_{n}^{\prime}\right)$ is cohomology of the complex $\left(\mathrm{C}^{\prime}\right)^{m}=\operatorname{Hom}_{s_{+} \operatorname{Mod}\left(R_{*}\right)}\left(\Omega(R / k)_{*} \otimes \Delta^{n}, G_{\partial}^{m} R_{*}\right)$.

Now, the canonical map $E^{\prime} \rightarrow E$ gives quasi-isomorphisms $E_{n}^{\prime} \rightarrow E_{n}$ for all $n$. We know that $E$ automatically satisfies (Q1). Since $\Omega\left(R_{*} / k\right)$ is a cofibrant $R_{*}$-module, the tangent space $\mathrm{C}\left(E^{\prime}\right)^{n}=\underline{\operatorname{Hom}}_{s_{+} \operatorname{Mod}\left(R_{*}\right)}\left(\Omega\left(R_{*} / k\right), G_{\partial}^{n} R_{*}\right)$ is trivially fibrant, so $E^{\prime}$ also satisfies (Q1).

It only remains to show that $E^{\prime}$ satisfies (Q2'); the proof of Proposition 4.16 adapts.

Remark 4.20. We may weaken the condition that $R_{\bullet}$ be cofibrant to requiring that the cotangent complex diag $\mathbb{L}_{\bullet}\left(R_{\bullet} / k\right)$ of $R_{\bullet}$ is equivalent in $\operatorname{Ho}\left(s \operatorname{Mod}\left(R_{\bullet}\right)\right)$ to $\Omega\left(R_{\bullet} / k\right)$, and that the latter is cofibrant. If a $k$-algebra $R$ (with constant simplicial structure) is smooth, [Ill] Proposition III.3.1.2 implies that this holds.

Definition 4.21. Given a simplicial $k$-algebra $R_{\bullet}$, and a simplicial $R_{\bullet}$-module $M_{\bullet}$, define the simplicial vector space $\underline{\operatorname{Der}}(R, M)$ of derived derivations by

$$
\underline{\operatorname{Der}}\left(R_{\bullet}, M_{\bullet}\right)_{n}:=\operatorname{Der}\left(R_{\bullet} \otimes \Delta^{n}, M_{\bullet}\right),
$$

the set of simplicial $k$-algebra morphisms $f: R_{\bullet} \otimes \Delta^{n} \rightarrow R_{\bullet} \oplus M_{\bullet} \epsilon$ extending the canonical map $R_{\bullet} \otimes \Delta^{n} \rightarrow R_{\bullet}$, where $\epsilon^{2}=0$.

Remark 4.22. For $R_{\bullet} \in s \mathrm{Alg}(k)$ cofibrant, and $E$ as in Proposition 4.19, $\mathrm{H}^{n}(E)=$ $\pi_{-n} \underline{\operatorname{Der}}_{s \mathrm{Alg}}\left(R_{\bullet}, R_{\bullet}\right)$ for $n \leq 0$. For $n>0, \mathrm{H}^{n}(E)$ is the $n$th cohomology of the cosimplicial complex

$$
C^{n}:=\operatorname{Der}_{s \operatorname{Alg}}\left(\mathcal{L}_{\partial}^{n+1} R_{\bullet}, R_{\bullet}\right)
$$

associated to the comonad $\mathcal{L}_{\partial}$ of Definition 4.13.

Proposition 4.23. If $R_{\bullet} \rightarrow R$ is a cofibrant resolution of a $k$-algebra $R$, then the DDC $E$ of Proposition 4.19 is quasi-isomorphic to DF, for $F$ the $S D C$

$$
F^{n}=\operatorname{Hom}_{\text {Mod }}\left(\operatorname{Symm}^{n} \tilde{M}, \tilde{M}\right)
$$

from [Pri1] §1.2.1, and $\tilde{M} \in \operatorname{FMod}(\Lambda)$ lifting the $k$-module $M$ underlying $R$.

Proof. By Proposition 3.28, we may assume that $R_{\bullet}$ is the standard resolution $R_{n}=$ $\perp^{n+1} R$, with $\tilde{R}_{n}=\operatorname{Symm}^{n+1}(\tilde{M})$.

Then we have $E$ quasi-isomorphic to the DDC $E^{\prime}$ given by

$$
\left(E^{\prime}\right)_{K}^{n}=\operatorname{Hom}_{s_{+} \operatorname{Alg}}\left(K_{*} \otimes \tilde{R}_{*}, G_{\partial}^{n} \tilde{R}_{*}\right)=\operatorname{Hom}_{s_{+} \operatorname{Mod}}\left(K_{*} \otimes \operatorname{Symm}^{*} \tilde{R}, G_{\partial}^{n} \tilde{R}_{*}\right) .
$$

The augmentation $\varepsilon: \tilde{R}_{*} \rightarrow \tilde{R}$ in $s_{+} \operatorname{Mod}(\Lambda)$ gives us a map

$$
\begin{aligned}
\chi:\left(E^{\prime}\right)_{K}^{n} \rightarrow \operatorname{Hom}_{s_{+} \operatorname{Mod}}\left(K_{*} \otimes \operatorname{Symm}^{*} \tilde{R}, G_{\partial}^{n} \tilde{R}\right) & =\operatorname{Hom}_{s_{+} \operatorname{Mod}}\left(\mathcal{L}^{n}\left(K_{*} \otimes \operatorname{Symm}^{*} \tilde{R}\right), \tilde{R}\right) \\
& =\operatorname{Hom}_{\text {Mod }}\left(K_{n} \otimes \operatorname{Symm}^{n} \tilde{R}, \tilde{R}\right) .
\end{aligned}
$$

But this is just $(D F)_{K}^{n}$, and it is straightforward to check that $\chi: E^{\prime} \rightarrow D F$ respects all the SDC operations.

Since $R_{\bullet} \rightarrow R$ is a resolution, we get a weak equivalence

$$
\mathrm{Z}^{n} \mathrm{C}^{*}(E)=\mathrm{Z}^{n} \underline{\operatorname{Der}}_{s \mathrm{Alg}}\left(R_{\bullet}, G^{*+1} R_{\bullet}\right) \rightarrow \mathrm{Z}^{n} \underline{\operatorname{Der}}_{s \mathrm{Alg}}\left(R_{\bullet}, G^{*+1} R\right)
$$


Now by Lemma 4.15,

$$
\begin{aligned}
\underline{\operatorname{Der}}_{s \mathrm{Alg}}\left(R_{\bullet}, G^{n+1} R\right)_{m} & =\operatorname{Der}_{\mathrm{Alg}}\left(\pi_{0} \mathcal{L}^{n+1}\left(\Delta^{m} \otimes R \bullet\right), R\right) \\
& =\operatorname{Der}_{\mathrm{Alg}}\left(R_{n}, R\right)^{\Delta_{n}^{m}} \\
& =\operatorname{Der}_{\mathrm{Alg}}\left(\operatorname{Symm}^{n} R, R\right)^{\Delta_{n}^{m}} \\
& =\mathrm{C}^{n}(D F)_{m} .
\end{aligned}
$$

Since $\mathrm{C}^{\bullet}(E)$ is quasi-smooth, $\left.\mathrm{H}^{n-i}(\lrcorner E\right)=\pi_{i} \mathrm{Z}^{n} \mathrm{C}^{*}(E)$, so

$$
\left.\left.\mathrm{H}^{*}(\lrcorner E\right) \cong \mathrm{H}^{*}(\lrcorner D F\right)=\mathrm{H}^{*}(F),
$$

which is just André-Quillen cohomology $\mathbb{D}_{k}^{*}(R, R)$, and so $\chi$ is a quasi-isomorphism.

Remark 4.24. Propositions 4.19 and 4.23 together imply that derived deformations of a $k$-algebra $R$ are equivalent to derived deformations of the operation $\partial_{0}$ on any cofibrant resolution $R_{\bullet} \rightarrow R$.

\section{Deformations of Artin stacks and simplicial schemes}

The problem we now wish consider is that of deforming of an algebraic stack $\mathfrak{X}$. We may take a smooth simplicial hypercovering $X_{\bullet} \rightarrow \mathfrak{X}$, with each $X_{n}$ a disjoint union of affine schemes (similarly to the proof of [Ols1] Theorem 11.1), and our first step will be to consider derived deformations of $X_{\bullet}$.

5.1. Cosimplicial algebras. Let $X$ • be a simplicial affine scheme. Equivalently, we may consider the cosimplicial algebra $[n] \mapsto \Gamma\left(X_{n}, \mathscr{O}_{X_{n}}\right)$.

Definition 5.1. The categories $c \mathrm{FMod}(A), c \mathrm{FAlg}(A), c_{+} \mathrm{FMod}(A)$, and $c_{+} \operatorname{FAlg}(A)$ (as given in Definition 4.9) can be made into simplicial categories (i.e. enriched in simplicial sets) by setting $\left(S^{K}\right)^{n}:=\left(S^{n}\right)^{K_{n}}$ for $K \in \mathbb{S}$, with structure maps $\left(S^{K}\right)(f)=S(f)^{K_{n}}$ 。 $K(f)^{*}:\left(S^{m}\right)^{K_{m}} \rightarrow\left(S^{n}\right)^{K_{n}}$, for morphisms $f$ in $\Delta$. We then define the simplicial $\underline{\text { Hom }}$ functor by

$$
\underline{\operatorname{Hom}}(R, S)_{n}:=\operatorname{Hom}\left(R, S^{\Delta^{n}}\right) .
$$

There is the following diagram of monadic adjunctions of functors $\mathcal{C}_{\Lambda} \rightarrow s$ Cat:

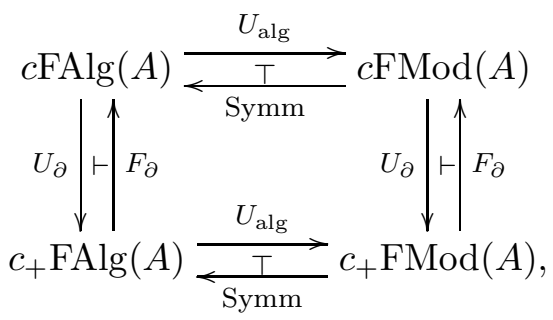

where $F_{\partial}: c_{+} \operatorname{FMod}(A) \rightarrow c \operatorname{FMod}(A)$ is left adjoint to the forgetful functor $U_{\partial}$, given by

$$
\left(F_{\partial} V^{*}\right)^{n}=V^{n} \oplus V^{n-1} \oplus \ldots \oplus V^{0},
$$

with operations dual to those in Lemma 4.12. Similarly, $F_{\partial}: c_{+} \mathrm{FAlg}(A) \rightarrow c \mathrm{FAlg}(A)$ is the left adjoint given by

$$
\left(F_{\partial} R^{*}\right)^{n}=R^{n} \otimes R^{n-1} \otimes \ldots \otimes R^{0} .
$$

The diagram satisfies the following commutativity conditions:

$$
U_{\partial} U_{\text {alg }}=U_{\text {alg }} U_{\partial} \quad \text { Symm } F_{\partial}=F_{\partial} \text { Symm, } \quad U_{\partial} \mathrm{Symm}=\operatorname{Symm} U_{\partial} .
$$

These adjunctions combine to give a monadic adjunction

$$
c \mathrm{FAlg}\left(A \underset{\mathrm{SymmF}_{\partial}}{\stackrel{U_{\partial} U_{\mathrm{alg}}}{\stackrel{T}{\rightleftarrows}}} c_{+} \mathrm{FMod}(A) .\right.
$$


Lemma 5.2. $c_{+} \operatorname{FMod}(A)$ has uniformly trivial deformation theory.

Proof. This is essentially the same as Proposition 4.16.

Proposition 5.3. In the scenario above, Theorem 3.13 gives a pre-DDC

$$
E^{n}:=\underline{\operatorname{Hom}}_{c_{+} \text {FMod }}\left(\top^{n} \widehat{U_{\partial} U_{\mathrm{alg}}} R, \widetilde{U_{\partial} U_{\mathrm{alg}}} R\right)
$$

satisfying Definition 2.8.(Q1), where $\top=U_{\partial} U_{\text {alg }} \mathrm{Symm} F_{\partial}$.

We now seek conditions under which the pre-DDC $E$ (or similarly a pre-DDC $E(\mathbb{D})$ associated to a diagram as in $\S 3.3)$ is quasi-smooth.

Definition 5.4. Given a cosimplicial (resp. almost cosimplicial) $A$-algebra $R$, define the category $c \operatorname{Mod}(R)\left(\right.$ resp. $\left.c_{+} \operatorname{Mod}(R)\right)$ to consist of cosimplicial (resp. almost cosimplicial) $A$-modules $M$ equipped with an associative multiplication $R \otimes M \rightarrow M$, respecting the cosimplicial (resp. almost cosimplicial) structures. These categories have simplicial structures, with $\left(M^{K}\right)^{n}:=\left(M^{n}\right)^{K_{n}}$, for $K \in \mathbb{S}$, the $R$-module structure on $M^{K}$ coming from the map $R \rightarrow R^{K}$. As usual, denote the left adjoint to $M \mapsto M^{K}$ by $N \mapsto N \otimes K$.

Given $M \in c \operatorname{Mod}(R)$ and an injective $\operatorname{map} K \hookrightarrow L$ in $\mathbb{S}$, set $M \otimes(L / K):=\operatorname{coker}(M \otimes$ $K \rightarrow M \otimes L)$ and $M^{L / K}:=\operatorname{ker}\left(M^{L} \rightarrow M^{K}\right)$.

Definition 5.5. Let $\perp_{\text {alg }}=\operatorname{Symm} U_{\text {alg }}, \perp_{\partial}=F_{\partial} U_{\partial}$ and $\perp=\operatorname{Symm} F_{\partial} U_{\partial} U_{\text {alg }}=\perp_{\partial} \perp_{\text {alg }}$. Given $R \in c \operatorname{Alg}(k)$, define $\mathbb{L}_{n}^{\perp}(R) \in c \operatorname{Mod}(R)$ by the property that

$$
\operatorname{Hom}_{c \operatorname{Mod}(R)}\left(\mathbb{L}_{n}^{\perp}(R), M^{\bullet}\right) \cong \operatorname{Der}_{k}\left(\perp^{n+1} R, M^{\bullet}\right)
$$

functorial in $M^{\bullet} \in c \operatorname{Mod}(R)$. Here, $\operatorname{Der}_{k}\left(S^{\bullet}, M^{\bullet}\right)$ is the set of morphisms $f: S^{\bullet} \rightarrow$ $S^{\bullet} \oplus M^{\bullet} \epsilon$ in $c \operatorname{Alg}_{k}$ extending the identity, where $\epsilon^{2}=0$.

Define $\mathbb{L}_{n}(R) \in c \operatorname{Mod}(R)$ by

$$
\operatorname{Hom}_{c \operatorname{Mod}(R)}\left(\mathbb{L}_{n}(R), M^{\bullet}\right) \cong \operatorname{Der}_{k}\left(\perp_{\text {alg }}^{n+1} R, M^{\bullet}\right) .
$$

Observe that $\mathbb{L}_{\bullet}(R)$ and $\mathbb{L}_{\bullet}^{\perp}(R)$ both form simplicial complexes in $c \operatorname{Mod}(R)$.

Definition 5.6. Given an object $R$ of $c \mathrm{FAlg}(A)$ (resp. $c_{+} \mathrm{FAlg}(A)$ ), we may extend $R$ uniquely to a cocontinuous functor $R: \mathbb{S} \rightarrow \operatorname{FAlg}(A)$ (resp. $R: s_{+} \operatorname{Set} \rightarrow \operatorname{FAlg}(A)$ ) extending the functor $R: \Delta \rightarrow \mathrm{FAlg}(A)$ (resp. $R: \Delta_{+} \rightarrow \mathrm{FAlg}(A)$ ) given by $R\left(\Delta^{n}\right)=R^{n}$ (resp. $R\left(\Xi^{n}\right)=R^{n}$, for $\Xi$ as in Definition 4.14).

Lemma 5.7. For all $m$, the simplicial complex $\mathbb{L}_{\bullet}^{\perp}(R)^{m}$ is a model for the cotangent complex of $R^{m}$.

Proof. Write $\perp_{n} R:=\perp^{n+1} R$; these form a simplicial complex $\perp_{\bullet} R$ in $c \operatorname{Alg}(k)$. We need to show that $\left(\perp_{\bullet} R\right)^{m}$ is a cofibrant resolution of $R^{m}$ in $s \operatorname{Alg}(k)$. If we apply the forgetful functor $U_{\partial} U_{\text {alg }}$ to the augmented simplicial complex $\perp_{\bullet} R \rightarrow R$, we see that it becomes contractible. In particular, this implies that $\perp_{\bullet} R \rightarrow R$ is contractible as an augmented complex of $k$-vector spaces, so it is a resolution.

Lemma 5.8. $\mathbb{L}_{n}^{\perp}(R)$ is a projective object of $c \operatorname{Mod}(R)$, and $U_{\partial} \mathbb{L}_{n}(R), U_{\partial} \mathbb{L}_{n}^{\perp}(R)$ are both projective objects of $c_{+} \operatorname{Mod}\left(R^{*}\right)$.

Proof. By adjointness,

$$
\operatorname{Der}_{k}\left(\perp^{n+1} R, M^{\bullet}\right) \cong \operatorname{Hom}_{C_{+} \operatorname{Mod}_{k}}\left(U_{\partial} U_{\text {alg }} \perp^{n} R, M^{*}\right),
$$

so $\operatorname{Der}_{k}\left(\perp^{n+1} R,-\right)$ defines a right exact functor, hence $\mathbb{L}_{n}^{\perp}(R)$ is projective. The other results follow similarly.

Lemma 5.9. There is a natural transformation $F_{\partial} U_{\text {alg }} \rightarrow U_{\text {alg }} F_{\partial}$, giving transformations $\perp_{\partial} \perp_{\text {alg }} \rightarrow \perp_{\text {alg }} \perp_{\partial}$. 
Proof. The transformation is given on level $n$ by

$$
R^{0} \oplus R^{1} \oplus \ldots \oplus R^{n} \ni \sum_{i=0}^{n} r_{i} \mapsto \sum_{i=0}^{n} 1 \otimes \ldots \otimes 1 \otimes r_{i} \otimes 1 \otimes \ldots 1 \in R^{0} \otimes R^{1} \otimes \ldots \otimes R^{n} .
$$

Definition 5.10. A morphism $f: X_{\bullet} \rightarrow Y_{\bullet}$ of simplicial schemes over $A$ is said to be quasi-smooth (resp. trivially smooth) if the morphism

$$
\operatorname{Hom}_{\mathbb{S}}\left(L, X_{\bullet}\right) \rightarrow \operatorname{Hom}_{\mathbb{S}}\left(K, X_{\bullet}\right) \times_{\operatorname{Hom}_{\mathbb{S}}\left(K, Y_{\bullet}\right)} \operatorname{Hom}_{\mathbb{S}}\left(L, Y_{\bullet}\right)
$$

of affine schemes is smooth for all trivial cofibrations (resp. all cofibrations) $K \rightarrow L$ of finite simplicial sets. The map $f$ is said to be smooth if it is quasi-smooth and $f_{0}: X_{0} \rightarrow Y_{0}$ is smooth.

We say that a morphism $R^{\bullet} \rightarrow S^{\bullet}$ in $c \operatorname{Alg}(A)$ is quasi-smooth (resp. trivially smooth, resp. smooth) if $\operatorname{Spec} S^{\bullet} \rightarrow \operatorname{Spec} R^{\bullet}$ is so.

Lemma 5.11. In Definition 5.10, we may replace cofibrations (resp. trivial cofibrations) $K \rightarrow L$ by generating cofibrations $\partial \Delta^{n} \rightarrow \Delta^{n}$ (resp. generating trivial cofibrations $\Lambda_{k}^{n} \rightarrow$ $\left.\Delta^{n}\right)$.

Proof. This follows because every cofibration (resp. trivial cofibration) is a composition of pushouts of generating cofibrations (resp. generating trivial cofibrations), and the fact that smooth morphisms are closed under pullback and finite composition.

Lemma 5.12. A morphism $f: X_{\bullet} \rightarrow Y_{\bullet}$ of simplicial schemes is quasi-smooth (resp. trivially smooth, resp. smooth) if and only if the following conditions hold:

(1) for all square-zero extensions $A \rightarrow B$ of $k$-algebras, the map $X_{\bullet}(A) \rightarrow$ $X_{\bullet}(B) \times_{Y_{\bullet}(B)} Y_{\bullet}(A)$ is a fibration (resp. a trivial fibration, resp. a surjective fibration) in $\mathbb{S}$.

(2) for all all vertices $v \in \Delta_{0}^{n}$ the maps $v^{*}: X_{n} \rightarrow X_{0}$ (resp. the schemes $X_{n}$, resp. the schemes $X_{n}$ ) are locally of finite presentation.

Proof. This follows from the fact that a morphism is smooth if and only if it is quasismooth and locally of finite presentation, and that $U \rightarrow V$ is locally of finite presentation if and only if the map $U\left(A_{\alpha}\right) \rightarrow U\left(\lim _{\longrightarrow} A_{\alpha}\right) \times_{V\left(\underline{\lim } A_{\alpha}\right)} \underset{\lim }{\longrightarrow} V\left(A_{\alpha}\right)$ is an isomorphism. We also use the result that if $g \circ f$ is locally of finite presentation, then $f$ must also be so.

Corollary 5.13. For all cofibrations $i: K \rightarrow L$ of finite simplicial sets, and $f: X \rightarrow Y$ a quasi-smooth morphism of simplicial affine schemes, the map

$$
g: X^{L} \rightarrow X^{K} \times_{Y^{K}} Y^{L}
$$

is quasi-smooth. Moreover, if either $i$ or $f$ is trivial, then so is $g$.

Lemma 5.14. If $R \rightarrow S$ is a trivially smooth map in cAlg, then $\Omega(S / R)$ is projective in $c \operatorname{Mod}(S)$.

Proof. By definition, we know that $R(L) \otimes_{R(K)} S(K) \rightarrow S(L)$ is smooth for all cofibrations $K \hookrightarrow L$ of finite simplicial sets.

Given $M^{\bullet} \in c \operatorname{Mod}(S)$ and $K \in \mathbb{S}$, define $M(K) \in \operatorname{Mod}(S(K))$ by $S(K) \oplus M(K) \epsilon=$ $(S \oplus M \epsilon)(K)$. Note that $\Omega(S / R)(K)=\Omega(S(K) / R(K))$.

Take a surjection $L^{\bullet} \rightarrow N^{\bullet}$ in $c \operatorname{Mod}(S)$ and a morphism $f: \Omega(S / R) \rightarrow N^{\bullet}$. We will construct a lifting $\tilde{f}$ of $f$ inductively. Assume that we have $R^{m}$-linear maps $\tilde{f}^{m}$ : $\Omega(S / R)^{m} \rightarrow L^{m}$ lifting $f$ compatibly with the cosimplicial operations, for all $m<n$. If 
$M^{n} L$ denotes the $m$ th matching object (as in [GJ] Lemma VII.4.9), then extending $\tilde{f}$ compatibly to $\Omega(S / R)^{n}$ amounts to finding a lift

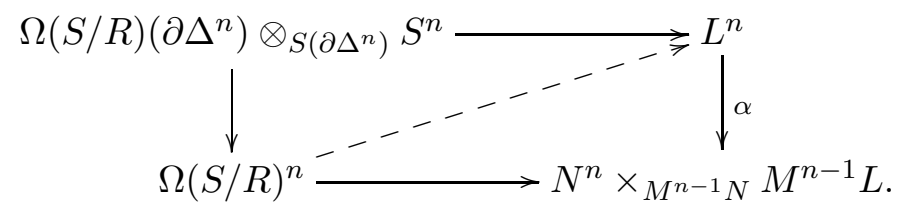

Now, $T:=R^{n} \otimes_{R\left(\partial \Delta^{n}\right)} S\left(\partial \Delta^{n}\right) \rightarrow S^{n}$ is smooth, as is $R(K) \rightarrow S(K)$ for all $K$. Thus the sequence

$$
0 \rightarrow \Omega\left(S\left(\partial \Delta^{n}\right) / R\left(\partial \Delta^{n}\right)\right) \otimes_{S\left(\partial \Delta^{n}\right)} S^{n} \rightarrow \Omega\left(S^{n} / R^{n}\right) \rightarrow \Omega\left(S^{n} / T\right) \rightarrow 0
$$

is exact, with all terms projective. Since $\alpha$ is surjective, projectivity of $\Omega\left(S^{n} / T\right)$ gives the required lift.

Lemma 5.15. If $R$ is quasi-smooth, then for all trivial cofibrations $K \rightarrow L$ of finite simplicial sets, $\Omega(R) \otimes(L / K)$ is projective in $c \operatorname{Mod}(R)$.

Proof. By Corollary 5.13, $R \otimes K \rightarrow R \otimes L$ is trivially smooth. Since $\Omega(R) \otimes X=\Omega(R \otimes$ $X) \otimes_{R \otimes X} R$, projectivity follows from Lemma 5.14.

Definition 5.16. Let $\mathcal{L}^{\vee}: c_{+} \operatorname{Mod} \rightarrow c$ Mod be right adjoint to $U_{\partial}$.

Lemma 5.17. For $R \in c \mathrm{Alg}(k)$ and an injective map $f: Z \rightarrow X$ in $s_{+}$Set, there is an isomorphism

$$
\mathrm{H}^{i} \operatorname{Hom}_{c_{+} \operatorname{Mod}\left(R^{*}\right)}\left(\mathbb{L}_{\bullet}\left(R^{*}\right), N \otimes k^{X / Z}\right) \cong \mathbb{E x t}_{c \operatorname{Mod}(R)}^{i}\left(\mathbb{L}_{\bullet}^{\perp}(R) \otimes(\mathcal{L} X / \mathcal{L} Z), \mathcal{L}^{\vee} N\right)
$$

for all $N \in c_{+} \operatorname{Mod}\left(R^{*}\right)$.

Proof. By Lemma 5.8, $\mathbb{L}_{n}^{\perp}(R)$ is projective, so $\mathbb{L}_{n}^{\perp}(R) \otimes(\mathcal{L} X / \mathcal{L} Z)$ must also be projective, as $M \mapsto M^{K}$ sends surjections to surjections. Thus

$$
\begin{aligned}
\mathbb{E x t}_{c \operatorname{Mod}(R)}^{*}\left(\mathbb{L}_{\bullet}^{\perp}(R) \otimes(\mathcal{L} X / \mathcal{L} Z), \mathcal{L}^{\vee} N\right) & =\mathrm{H}^{*} \operatorname{Hom}_{c \operatorname{Mod}(R)}\left(\mathbb{L}_{\bullet}^{\perp}(R) \otimes(\mathcal{L} X / \mathcal{L} Z), \mathcal{L}^{\vee} N\right) \\
& =\mathrm{H}^{*} \operatorname{Hom}_{c \operatorname{Mod}(R)}\left(\mathbb{L}_{\bullet}^{\perp}(R), \mathcal{L}^{\vee} N \otimes \mathcal{L}^{\vee} k^{X / Z}\right) \\
& =\mathrm{H}^{*} \operatorname{Hom}_{c_{+} \operatorname{Mod}\left(R^{*}\right)}\left(U_{\partial} \mathbb{L}_{\bullet}^{\perp}(R), N \otimes k^{X / Z}\right) \\
& =\mathbb{E x t}_{c+\operatorname{Mod}\left(R^{*}\right)}\left(U_{\partial} \mathbb{L}_{\bullet}^{\perp}(R), N \otimes k^{X / Z}\right) .
\end{aligned}
$$

Now, Lemma 5.9 gives compatible transformations $\perp_{\partial}^{n+1} \perp_{\text {alg }}^{n+1}(R) \rightarrow \perp^{N+1} R$. The unit of the adjunction $F_{\partial} \dashv U_{\partial}$ gives compatible transformations $U_{\partial} \rightarrow U_{\partial} \perp_{\partial}^{n+1}$, so there is a map $\mathbb{L}_{\bullet}\left(R^{*}\right)=U_{\partial} \mathbb{L}_{\bullet}\left(R^{\bullet}\right) \rightarrow U_{\partial} \mathbb{L}_{\bullet}^{\perp}(R)$, which is an equivalence in the derived category by Lemma 5.7. Hence

$$
\begin{aligned}
\mathbb{E x t}_{c_{+} \operatorname{Mod}\left(R^{*}\right)}^{*}\left(U_{\partial} \mathbb{L}_{\bullet}^{\perp}(R), N \otimes k^{X / Z}\right) & \cong \mathbb{E x t}_{c_{+} \operatorname{Mod}\left(R^{*}\right)}^{*}\left(\mathbb{L}_{\bullet}\left(R^{*}\right), N \otimes k^{X / Z}\right) \\
& =\mathrm{H}^{*} \operatorname{Hom}_{c_{+} \operatorname{Mod}\left(R^{*}\right)}\left(\mathbb{L}_{\bullet}\left(R^{*}\right), N \otimes k^{X / Z}\right) .
\end{aligned}
$$

Lemma 5.18. For $R \in c \operatorname{Alg}(k)$ quasi-smooth, there is an exact sequence

$$
0 \rightarrow \mathbb{L}_{\bullet}\left(R^{0}\right) \otimes_{R^{0}} R^{*} \rightarrow \mathbb{L}_{\bullet}\left(R^{*}\right) \rightarrow \Omega\left(R^{*} / R^{0}\right) \rightarrow 0
$$

in the derived category of projective complexes in $c_{+} \operatorname{Mod}\left(R^{*}\right)$, where the morphism $R^{0} \rightarrow$ $R^{*}$ is given in level $n$ by $\left(\partial^{1}\right)^{n}$.

Proof. There is an exact sequence

$$
0 \rightarrow \mathbb{L}_{\bullet}\left(R^{0}\right) \otimes_{R^{0}} R^{*} \rightarrow \mathbb{L}_{\bullet}\left(R^{*}\right) \rightarrow \mathbb{L}\left(R^{*} / R^{0}\right) \rightarrow 0
$$

in the derived category. Since $R^{\bullet}$ is quasi-smooth, the maps $\left(\partial^{1}\right)^{n}: R^{0} \rightarrow R^{n}$ are all smooth, giving $\mathbb{L}\left(R^{*} / R^{0}\right) \sim \Omega\left(R^{*} / R^{0}\right)$ 
Proposition 5.19. If $R$ is a quasi-smooth object of $c \operatorname{Alg}(k)$, then every morphism $\rho: R \rightarrow$ $S$ in $c \mathrm{Alg}$ is quasi-smooth over $c_{+} \mathrm{Mod}$, in the sense of Definition 3.22. The Ext-groups are then given by

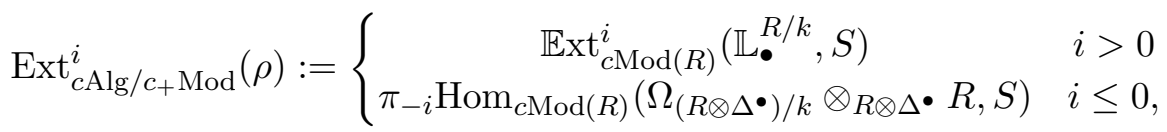

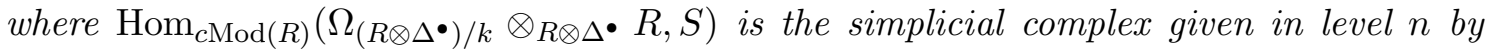
$\operatorname{Hom}_{c \operatorname{Mod}(R)}\left(\Omega_{\left(R \otimes \Delta^{n}\right) / k} \otimes_{R \otimes \Delta^{n}} R, S\right)$.

Proof. First observe that since $c_{+}$Mod has uniformly trivial deformation theory, $\rho$ is quasismooth over $c_{+}$Mod whenever it is Q2 over $c_{+}$Mod. Now,

$$
\mathrm{C}_{c \mathrm{Alg} / c_{+} \operatorname{Mod}}(\rho)=\underline{\operatorname{Hom}}_{c \operatorname{Mod}(R)}\left(\mathbb{L}_{\bullet}^{\perp}(R), S\right),
$$

so for $K \in \mathbb{S}$,

$$
\begin{aligned}
\underline{\operatorname{Ext}}_{c \mathrm{Alg} / c_{+} \operatorname{Mod}}^{*}(\rho) & =\mathrm{H}^{*} \underline{\operatorname{Hom}}_{c \operatorname{Mod}(R)}\left(\mathbb{L}_{\bullet}^{\perp}(R), S\right), \\
\underline{\operatorname{Ext}}_{c \operatorname{Alg} / c_{+} \operatorname{Mod}}(\rho)_{K} & =\mathrm{H}^{*} \operatorname{Hom}_{c \operatorname{Mod}(R)}\left(\mathbb{L}_{\bullet}^{\perp}(R) \otimes K, S\right), \\
& \cong \mathbb{E x t}_{c \operatorname{Mod}(R)}^{*}\left(\mathbb{L}_{\bullet}^{\perp}(R) \otimes K, S\right),
\end{aligned}
$$

the latter isomorphism following since $\mathbb{L}_{n}^{\perp}(R) \otimes K$ is projective.

Now, consider the monad $T_{\partial}:=\mathcal{L}^{\vee} U_{\partial}$ on $c \operatorname{Mod}(R)$, and observe that the augmented cosimplicial complex $\top_{\partial}^{\bullet+1} M$ given in level $n$ by $\top_{\partial}^{n+1} M$ is a resolution in $c \operatorname{Mod}(R)$, since it becomes contractible on applying $U_{\partial}$. Thus

$$
\underline{\operatorname{Ext}}_{c \mathrm{Alg} / c_{+} \operatorname{Mod}}^{*}(\rho)_{K} \cong \mathbb{E x t}_{c \operatorname{Mod}(R)}^{*}\left(\mathbb{L}_{\bullet}^{\perp}(R) \otimes K, \top_{\partial}^{\bullet+1} S\right) .
$$

Given $f: Z \rightarrow X$ in $s_{+}$Set with $f_{0}$ an isomorphism, by Lemmas 5.17 and 5.18 , we have

$$
\mathbb{E x t}_{c \operatorname{Mod}(R)}^{i}\left(\mathbb{L}_{\bullet}^{\perp}(R) \otimes(\mathcal{L} X / \mathcal{L} Z), \mathcal{L}^{\vee} N\right)=\mathrm{H}^{i} \operatorname{Hom}_{c_{+} \operatorname{Mod}\left(R^{*}\right)}\left(\mathbb{L}_{\bullet}\left(R^{0}\right) \otimes_{R^{0}} R^{*}, N \otimes k^{X / Z}\right)
$$

for all $i>0$. However,

$$
\operatorname{Hom}_{c_{+} \operatorname{Mod}\left(R^{*}\right)}\left(\mathbb{L}_{\bullet}\left(R^{0}\right) \otimes_{R^{0}} R^{*}, N \otimes k^{X / Z}\right)=\operatorname{Hom}_{\operatorname{Mod}\left(R^{0}\right)}\left(\mathbb{L}_{\bullet}\left(R^{0}\right),\left(N \otimes k^{X / Z}\right)^{0}\right)=0,
$$

since $\left(k^{X / Z}\right)^{0}=0$.

Hence $\mathbb{E x t}_{c \operatorname{Mod}(R)}^{i}\left(\mathbb{L}_{\bullet}^{\perp}(R) \otimes(\mathcal{L} X / \mathcal{L} Z), \mathcal{L}^{\vee} N\right)=0$ for all $i>0$, so the spectral sequence associated to $\top_{\partial}^{\bullet+1}$ gives

$$
\mathbb{E x t}_{c \operatorname{Mod}(R)}^{*}\left(\mathbb{L}_{\bullet}^{\perp}(R) \otimes(\mathcal{L} X / \mathcal{L} Z), M\right) \cong \mathrm{H}^{*} \operatorname{Hom}_{c \operatorname{Mod}(R)}\left(\Omega(R) \otimes(\mathcal{L} X / \mathcal{L} Z), \top_{\partial}^{\bullet+1} M\right) .
$$

Since $\Omega(R) \otimes(\mathcal{L} X / \mathcal{L} Z)$ is projective (by Lemma 5.14 ), this is just

$$
\begin{aligned}
\mathbb{E x t}_{c \operatorname{Mod}(R)}^{*}\left(\Omega(R) \otimes(\mathcal{L} X / \mathcal{L} Z), \top_{\partial}^{\bullet+1} M\right) & \cong \operatorname{Ext}_{c \operatorname{Mod}(R)}^{*}(\Omega(R) \otimes(\mathcal{L} X / \mathcal{L} Z), M) \\
& =\operatorname{Hom}_{c \operatorname{Mod}(R)}\left(\Omega(R \otimes \mathcal{L} X / R \otimes \mathcal{L} Z) \otimes_{R \otimes \mathcal{L} X} R, M\right) .
\end{aligned}
$$

Taking $Z=\partial \Xi^{n}, X=\Xi^{n}$, we have $\mathcal{L} Z=\Lambda_{0}^{n}, \mathcal{L} X=\Delta^{n}$, and

$$
N_{n} \underline{\operatorname{Ext}}_{c \mathrm{Alg} / c_{+} \operatorname{Mod}}^{*}(\rho)=\operatorname{Hom}_{c \operatorname{Mod}(R)}\left(\Omega\left(R \otimes \Delta^{n} / R \otimes \Lambda_{0}^{n}\right) \otimes_{R \otimes \Delta^{n}} R, S\right),
$$

so $\underline{\operatorname{Ext}}_{c \mathrm{Alg} / c_{+} \text {Mod }}^{i}(\rho)$ is constant for $i>0$.

Thus $\rho$ is Q2 over $c_{+}$Mod, as required. The description of positive Ext-groups follows from Lemma 5.17, while that of non-positive Ext-groups follows from the definition of $\pi_{-i} \mathrm{H}^{0} \mathrm{C}_{c \mathrm{Alg} / c_{+} \operatorname{Mod}}(\rho)$. 
Corollary 5.20. For any diagram in cAlg with quasi-smooth objects, the associated preDDC given by Definition 3.24 and Proposition 3.19 applied to the adjunction

$$
c \mathrm{FAlg}(A) \underset{\operatorname{Symm} F_{\partial}}{\stackrel{U_{\partial} U_{\mathrm{alg}}}{\underset{T}{T}} c_{+}} \operatorname{FMod}(A)
$$

is a DDC by Lemma 3.25, and governs deformations in the simplicial category cAlg (by Proposition 3.26).

5.1.1. Comparison with deformations of schemes. In [Pri2] §3.2.1, an SDC was constructed to describe deformations of a separated scheme $X$, and we now wish to compare it with the DDC above.

Take an open affine cover $\left(X_{\alpha}\right)_{\alpha \in I}$ of $X$, and set $\check{X}:=\coprod_{\alpha \in I} X_{\alpha}$. Define the simplicial scheme $Z \bullet$ by $Z=\operatorname{cosk}_{0}(\check{X} / X)$, i.e.

$$
Z_{n}=\overbrace{\check{X} \times_{X} \check{X} \times_{X} \ldots \times_{X} \check{X}}^{n+1}
$$

with $r_{n}: Z_{n} \rightarrow X$, and $s_{n}: Z_{n} \rightarrow \check{X}$ given by projection onto the first factor.

The map $v: \check{X} \rightarrow X$ gives adjoint functors $v^{-1} \dashv v_{*}$ on sheaves. This yields the following diagram of Cat-valued functors:

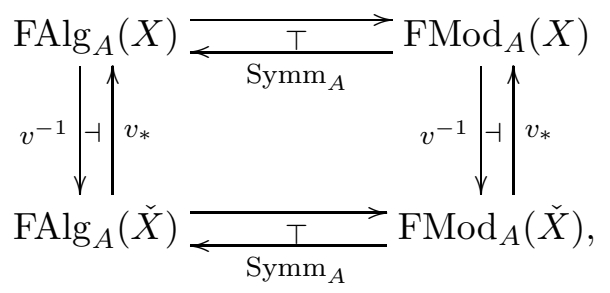

where $\operatorname{FMod}_{A}(Y)$ and $\mathrm{FAlg}_{A}(Y)$ denote sheaves of flat $A$-modules and of flat $A$-algebras on $Y$.

Definition 5.21. The SDC $\check{E}^{\bullet}$ of [Pri2] $§ 3.2 .1$ was then given by

$$
\check{E}^{n}(A)=\operatorname{Hom}_{\mathrm{FMod}_{A}(\check{X})}\left(\left(\operatorname{Symm}_{A}\right)^{n} \mathscr{N} \otimes A,\left(v^{-1} v_{*}\right)^{n} \mathscr{N} \otimes A\right)_{v^{-1}\left(\alpha^{n} \circ \varepsilon^{n}\right)},
$$

for $\mathscr{N}$ a flat $\mu$-adic $\Lambda$-module on $\check{X}$ lifting $v^{-1} \mathscr{O}_{X}$, with $\alpha^{n}: \mathscr{O}_{X} \rightarrow\left(v_{*} v^{-1}\right)^{n} \mathscr{O}_{X}$ coming from the unit of the adjunction, and similarly $\varepsilon^{n}:\left(\operatorname{Symm}_{k}\right)^{n} \mathscr{O}_{X} \rightarrow \mathscr{O}_{X}$.

Definition 5.22. Define functors $\check{C}^{\bullet}: \operatorname{FMod}_{A}(X) \rightarrow c \operatorname{FMod}(A), \check{C}^{*}: \operatorname{FMod}_{A}(\check{X}) \rightarrow$ $c_{+} \operatorname{FMod}(A)$ by

$$
\check{\mathrm{C}}^{n}(\mathscr{F}):=\Gamma\left(Z_{n}, r_{n}{ }^{-1} \mathscr{F}\right), \quad \check{\mathrm{C}}^{n}(\mathscr{G}):=\Gamma\left(Z_{n}, s_{n}{ }^{-1} \mathscr{G}\right),
$$

with the standard cosimplicial operations.

Lemma 5.23. There are canonical isomorphisms

$$
\mathrm{C}^{*}\left(v^{-1} \mathscr{F}\right)=U_{\partial} \mathrm{C}^{\bullet}(\mathscr{F}) \quad \mathrm{C}^{\bullet}\left(v_{*} \mathscr{G}\right) \cong \mathcal{L}^{\vee} \mathrm{C}^{*}(\mathscr{G}) .
$$

Lemma 5.24. There is a canonical natural transformation Symm $\circ \check{C}^{\bullet} \rightarrow \check{C}^{\bullet} \circ$ Symm.

Proposition 5.25. The $S D C \check{E}$ is quasi-isomorphic to the DDC E of Proposition 5.3, in the sense that $\mathfrak{D} \mathfrak{e} \mathfrak{f}(\check{E})$ and $\mathfrak{D} \mathfrak{e} \mathfrak{f}(E)$ are weakly equivalent (equivalently, $D \check{E}$ and $E$ are quasi-isomorphic DDCs). 
Proof. We have maps

$$
\begin{aligned}
\operatorname{Hom}_{\operatorname{Mod}(\check{X})}\left(\top_{\text {alg }}^{n} \mathscr{M},\left(v^{-1} v_{*}\right)^{n} \mathscr{M}\right) & \rightarrow \operatorname{Hom}_{c_{+} \operatorname{Mod}}\left(\check{\mathrm{C}}^{*}\left(\top_{\text {alg }}^{n} \mathscr{M}\right), \check{\mathrm{C}}^{*}\left(v^{-1} v_{*}\right)^{n} \mathscr{M}\right) \\
& =\operatorname{Hom}_{c_{+} \operatorname{Mod}}\left(\check{\mathrm{C}}^{*}\left(\top_{\text {alg }}^{n} \mathscr{M}\right),\left(\mathcal{L}^{\vee}\right)^{n} \check{\mathrm{C}}^{*} \mathscr{M}\right) \\
& =\operatorname{Hom}_{c_{+} \operatorname{Mod}}\left(\top_{\partial}^{n} \check{\mathrm{C}}^{*}\left(\top_{\text {alg }}^{n} \mathscr{M}\right), \check{\mathrm{C}}^{*} \mathscr{M}\right) \\
& \rightarrow \operatorname{Hom}_{c_{+} \operatorname{Mod}}\left(\top_{\text {alg }}^{n} \top_{\partial}^{n} \check{\mathrm{C}}^{*}(\mathscr{M}), \check{\mathrm{C}}^{*} \mathscr{\mathscr { M }}\right) \\
& \rightarrow \operatorname{Hom}_{c_{+} \operatorname{Mod}}\left(\top^{n} \check{\mathrm{C}}^{*}(\mathscr{M}), \check{\mathrm{C}}^{*} \mathscr{\mathscr { M }}\right) .
\end{aligned}
$$

These are compatible with the SDC operations, giving a morphism $\check{E} \rightarrow E_{0}$ of SDCs. Now, as in Proposition 5.19,

$$
\mathrm{H}^{*}\left(E_{0}\right)=\mathbb{E x t}_{\mathscr{O}_{Z}}^{*}\left(\mathbb{L}_{\bullet}^{Z / k}, \mathscr{O}_{Z}\right) .
$$

However, since the maps $r_{n}: Z_{n} \rightarrow X$ are all open, and hence étale, $\mathbb{L}_{\bullet}^{Z / k}$ is quasiisomorphic to $r^{*} \mathbb{L}_{\bullet}^{X}$. Thus

$$
\mathbb{E x t}_{Z_{\bullet}}^{*}\left(\mathbb{L}_{\bullet}^{Z / k}, \mathscr{O}_{Z}\right)=\mathbb{E x t}_{\mathscr{O}_{X}}^{*}\left(\mathbb{L}_{\bullet}^{X / k}, r_{*} \mathscr{O}_{Z}\right)=\mathbb{E x t}_{\mathscr{O}_{X}}^{*}\left(\mathbb{L}_{\bullet}^{X / k}, \mathscr{O}_{X}\right)
$$

since $r_{*} \mathscr{O}_{Z_{\bullet}}=r_{*} r^{-1} \mathscr{O}_{X}$ is a resolution of $\mathscr{O}_{X}$. This means that $\check{E} \rightarrow E_{0}$ is a quasiisomorphism of SDCs.

Finally, to see that $\mathfrak{D} \mathfrak{e f}\left(E_{0}\right) \rightarrow \mathfrak{D e f}(E)$ is a quasi-isomorphism, apply Lemma 2.24, noting that the strictly positive cohomology groups automatically agree. For $n \leq 0$,

$$
\mathrm{H}^{n} E=\mathrm{H}_{-n} \operatorname{Hom}_{\mathscr{O}_{Z}}\left(i^{*} \Omega_{Z^{\Delta}} \cdot, \mathscr{O}_{Z}\right),
$$

for $i: Z \rightarrow Z^{\Delta^{n}}$. However, $Z$ is quasi-étale (the analogous notion to quasi-smooth), so the vertex maps $a: Z^{\Delta^{n}} \rightarrow Z$ are trivially étale, and thus $\Omega_{Z^{\Delta^{n}}} \cong a^{*} \Omega_{Z}$, so $i^{*} \Omega_{Z^{\Delta}}=\Omega_{Z}$. Therefore $\mathrm{H}^{0} E=\mathrm{H}^{0} E_{0}=\operatorname{Hom}_{\mathscr{O}_{X}}\left(\Omega_{X}, \mathscr{O}_{X}\right)$, and $\mathrm{H}^{n} E=0$ for $n<0$.

5.2. Quasi-compact, quasi-separated stacks. Let $\mathfrak{X}$ be a quasi-compact, quasiseparated stack, with presentation $P: X_{0} \rightarrow \mathfrak{X}$, for $X_{0}$ affine, giving a simplicial algebraic space $\operatorname{cosk}_{0}^{\mathfrak{X}}\left(X_{0}\right)$ (as considered in $[\mathrm{Aok}] \S 3$ ). We may then take an étale hypercovering $X_{\bullet} \rightarrow \operatorname{cosk}_{0}^{\mathfrak{X}}\left(X_{0}\right)$, for $X_{\bullet}$ a simplicial affine scheme, and denote the composition by $P_{\bullet}: X_{\bullet} \rightarrow \mathfrak{X}$.

Lemma 5.26. Every smooth simplicial hypercovering is trivially smooth.

Proof. For a map $U_{\bullet} \rightarrow V_{\bullet}$ to be a smooth hypercovering says that the matching maps $U_{n} \rightarrow V_{n} \times_{M_{n} V} M_{n} U$ are all smooth surjections.

Lemma 5.27. The simplicial affine scheme $X_{\bullet}$ is quasi-smooth.

Proof. Write $Z_{\bullet}:=\operatorname{cosk}_{0}^{\mathfrak{X}}\left(X_{0}\right)$. Since $Z_{\bullet}=B \mathfrak{G}$, for $\mathfrak{G}$ the groupoid space $X_{0} \times \mathfrak{X} X_{0} \Longrightarrow X_{0}$, all higher partial matching maps of $Z_{\bullet}$ are isomorphisms. In other words, for any trivial cofibration $i: K \rightarrow L$ in $\mathbb{S}$ with $i_{0}: K_{0} \rightarrow L_{0}$ an isomorphism, the map

$$
i^{*}: M_{L}(Z) \rightarrow M_{K}(Z)
$$

is an isomorphism.

By [Aok] Theorem 2.1.5, $\mathfrak{G}$ has SQCS structure so the maps $X_{1} \rightarrow \operatorname{Hom}_{\mathbb{S}}\left(\Lambda_{k}^{1}, X_{\bullet}\right)$ are smooth surjections for both $k$. Thus $Z \bullet$ is quasi-smooth, by Lemma 5.11. Since $X \bullet \rightarrow Z$ • is trivially smooth, the result follows.

Remark 5.28. Similarly, every strongly quasi-compact $n$-geometric Artin stack $\mathfrak{X}$ gives rise to a quasi-smooth simplicial affine scheme $X_{\bullet}$, by [Pri6] Theorem 4.7. The statement of Proposition 5.29 will then carry over to this generality, taking $\mathbb{L}_{\mathfrak{X}}$ to be the cotangent complex of [Pri6] §7.1. 
5.2.1. Cohomology and the cotangent complex. Given any morphism $f: \mathfrak{Y} \rightarrow \mathfrak{X}$ of quasicompact, quasi-separated stacks, lifting to a morphism $f: Y_{\bullet} \rightarrow X_{\bullet}$ of simplicial affine resolutions, in this section we will describe the Ext-groups

$$
\operatorname{Ext}_{c \mathrm{Alg} / c_{+} \operatorname{Mod}}^{*}\left(f^{\sharp}\right)
$$

of Proposition 5.19 in terms of the cotangent complex of [Ols1] $\S 8$. Ext-groups of the cotangent complex are defined in [Ols2] $§ 2.11$.

Let $\mathfrak{X}, X$ • be as above, and let $\mathscr{J}$ be a quasi-coherent sheaf on $\mathfrak{X}$. Since the cotangent complex $\mathbb{L}_{\mathfrak{X}}$ is in degrees $\geq-1$, we have $\operatorname{Ext}^{i}\left(\mathbb{L}_{\mathfrak{X}}, \mathscr{J}\right)=0$ for all $i<-1$. Since $r: X_{\bullet} \rightarrow$ $\operatorname{cosk}_{0}^{\mathfrak{X}}\left(X_{0}\right)$ is a hypercovering, the maps

$$
\mathrm{H}^{*}\left(\operatorname{cosk}_{0}^{\mathfrak{X}}\left(X_{0}\right), \mathscr{F}\right) \rightarrow \mathrm{H}^{*}\left(X_{\bullet}, r^{*} \mathscr{F}\right)
$$

on cohomology are isomorphisms for all quasi-coherent sheaves $\mathscr{F}$.

By [Aok] Proposition 3.4.2,

(1) $\operatorname{Ext}^{i}\left(\mathbb{L}_{\mathfrak{X}}, \mathscr{J}\right) \cong \operatorname{Ext}^{i}\left(\mathbb{L}_{X_{\bullet}}, P_{\bullet}^{*} \mathscr{J}\right)$ for $i>0$.

(2) $\operatorname{Ext}^{i}\left(\mathbb{L}_{\mathfrak{X}}, \mathscr{J}\right)=\mathrm{H}^{i}\left(\operatorname{Hom}\left(\Omega_{X_{0} / \mathfrak{X}}, P^{*} \mathscr{J}\right)[1] \rightarrow \operatorname{Hom}\left(\Omega_{X_{\bullet}}, P_{\bullet}^{*} \mathscr{J}\right)\right)$, for $i \leq 0$.

\section{Proposition 5.29.}

$$
\operatorname{Ext}_{c \mathrm{Alg} / c_{+} \operatorname{Mod}}^{i}\left(f^{\sharp}\right) \cong \operatorname{Ext}^{i}\left(\mathbb{L}_{\mathfrak{X}}, f_{*} \mathscr{O}_{\mathfrak{Y}}\right)
$$

for all $i \in \mathbb{Z}$.

Proof. For $i>0$, this is just the observation that $\operatorname{Ext}^{i}\left(\mathbb{L}_{X_{\bullet}}, P_{\bullet}^{*} \mathscr{J}\right)=\operatorname{Ext}_{c \mathrm{Alg} / c_{+} \operatorname{Mod}}^{i}\left(f^{\sharp}\right)$ when $\mathscr{J}=f_{*} \mathscr{O}_{\mathfrak{Y}}$.

Accordingly, we need to describe the non-positive Ext groups

$$
\mathrm{H}_{-i} \operatorname{Hom}_{X}\left(c^{*} \Omega_{X^{\Delta}} \bullet, \mathscr{J}\right)
$$

in terms of $\mathfrak{X}, X_{0}$, where $c: X \rightarrow X^{K}$ is the constant map.

Let $U_{\bullet}$ denote the simplicial complex $\operatorname{Hom}_{X}\left(c^{*} \Omega_{X^{\Delta}}, \mathscr{J}\right)$, and write $Z_{\bullet}:=\operatorname{cosk}_{0}^{\mathfrak{X}}\left(X_{0}\right)$, with $V_{\bullet}: \operatorname{Hom}_{Z}\left(c^{*} \Omega_{Z^{\Delta} \bullet}, \mathscr{J}\right)$. Since $X \rightarrow Z$ is trivially smooth, observe that the canonical map $U \rightarrow V$ is a trivial fibration, so $\mathrm{H}_{*}(U) \cong \mathrm{H}_{*}(V)$.

In general, if $K$ is contractible, then

$$
M_{K} Z=\overbrace{X_{0} \times \mathfrak{X} X_{0} \times \mathfrak{X} \ldots \times \mathfrak{X} X_{0}}^{K_{0}},
$$

so

$$
\Omega\left(M_{K} Z / \mathfrak{X}\right)=\bigoplus_{v \in K_{0}} v^{*} \Omega\left(X_{0} / \mathfrak{X}\right) .
$$

We therefore conclude that for a trivial cofibration $K \hookrightarrow L$,

$$
\Omega\left(M_{L} Z / M_{K} Z\right)=\bigoplus_{v \in L_{0}-K_{0}} v^{*} \Omega\left(X_{0} / \mathfrak{X}\right)
$$

so for $0: \bullet \rightarrow I$,

$$
\Omega\left(Z^{I} / Z\right)^{n}=\Omega\left(M_{I \times \Delta^{n}} Z / M_{\Delta^{n}} Z\right)=\bigoplus_{v \in \Delta_{0}^{n}}(v \times 1)^{*} \Omega\left(X_{0} / \mathfrak{X}\right),
$$

so

$$
i^{*} \Omega\left(Z^{I} / Z\right)^{n}=\bigoplus_{v \in \Delta_{0}^{n}} v^{*} \Omega\left(X_{0} / \mathfrak{X}\right)
$$

and

$$
\operatorname{Hom}_{\mathscr{O}(X)}\left(i^{*} \Omega\left(X^{I} / X\right), \mathscr{F}\right)=\operatorname{Hom}_{\mathscr{O}\left(X_{0}\right)}\left(\Omega\left(X_{0} / \mathfrak{X}\right), \mathscr{F}^{0}\right),
$$

giving

$$
N_{1} V=\operatorname{Hom}_{\mathscr{O}(X)}\left(i^{*} \Omega\left(X^{I} / X\right), f_{*} \mathscr{O}(Y)\right)=\operatorname{Hom}_{\mathscr{O}\left(X_{0}\right)}\left(\Omega\left(X_{0} / \mathfrak{X}\right), f_{*} \mathscr{O}\left(Y_{0}\right)\right) .
$$


Moreover, for $n \geq 2, \Lambda_{0}^{n} \rightarrow \Delta_{0}^{n}$ is an isomorphism, so $N_{n} V=0$.

Thus

$$
N V=\left(\operatorname{Hom}\left(\Omega_{X_{0} / \mathfrak{X}}, P^{*} \mathscr{J}\right)[-1] \rightarrow \operatorname{Hom}\left(\Omega_{X_{\bullet}}, P_{\bullet}^{*} \mathscr{J}\right)\right),
$$

as required.

\subsubsection{Comparing deformation groupoids.}

Definition 5.30. Given a small 2-category $\mathcal{C}$, define a simplicial category $B^{1} \mathcal{C}$ by setting $\mathrm{Ob}\left(B^{1} \mathcal{C}\right)=\mathrm{Ob}(\mathcal{C})$, and $\underline{\operatorname{Hom}}_{B^{1} \mathcal{C}}(x, y)=B \mathscr{H}_{0} m_{\mathcal{C}}(x, y)$, where $\mathscr{H}_{o m_{\mathcal{C}}}(x, y)$ is the 1category of homomorphisms from $x$ to $y$, and $B$ is the nerve functor.

Lemma 5.31. Given $x, y \in \mathrm{ObC}$ with $\mathscr{H}_{0 m_{\mathcal{C}}}(x, y)$ a groupoid, $\pi_{0} \underline{\operatorname{Hom}}_{B^{1} \mathcal{C}}(x, y)$ is the set of isomorphism classes in $\mathscr{H}_{0} m_{\mathcal{C}}(x, y)$, with $\pi_{1}\left(\underline{\operatorname{Hom}}_{B^{1} \mathcal{C}}(x, y), f\right)$ the set of 2 automorphisms of $f$, and $\pi_{i}\left(\underline{\operatorname{Hom}}_{B^{1} \mathcal{C}}(x, y), f\right)=0$ for $i>1$.

Definition 5.32. Define a 2-category structure on the category AlgGpdSp of algebraic groupoid spaces (as in [Aok]) by defining a 2-morphism $\eta$ between morphisms $f, f^{\prime}: G \rightarrow H$ by analogy with natural transformations. Explicitly, let $\mathrm{Ob} G$ be the space of objects of $G$, with Mor $G \rightarrow(\mathrm{Ob} G \times \mathrm{Ob} G)$ the space of isomorphisms, and similarly for $H$. We must have $\eta: \mathrm{Ob} G \rightarrow$ Mor $H$, with $s \circ \eta=f, t \circ \eta=f^{\prime}$, and the following diagram commuting

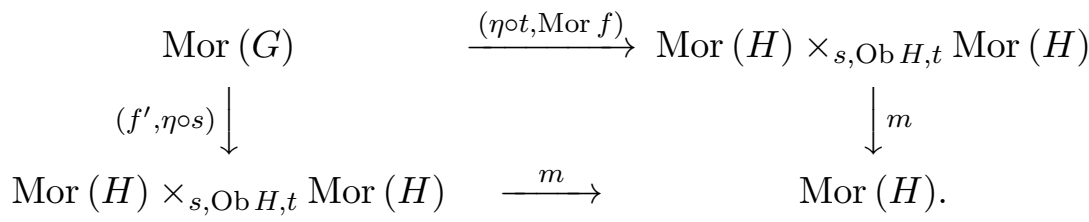

Definition 5.33. Given a 2-groupoid $\mathcal{G}$, define $\Pi_{0} \mathcal{G}$ to be the groupoid with objects Ob $\mathcal{G}$, and morphisms $\operatorname{Hom}_{\Pi_{0} \mathcal{G}}(X, Y)=\pi_{0} \mathscr{H}_{0} m_{\mathcal{G}}(X, Y)$. Similarly, for a simplicial groupoid $G_{\bullet}$, define $\Pi_{0} G_{\bullet}$ to be the groupoid with objects $\mathrm{Ob} G$, and morphisms $\operatorname{Hom}_{\Pi_{0}} \mathscr{G}_{\bullet}(X, Y)=$ $\pi_{0} \underline{\operatorname{Hom}}_{G}(X, Y)$.

Lemma 5.34. Given $G \in \mathrm{AlgGpdSp}$ associated to an algebraic stack over $k$, the nerve functor $B: \operatorname{AlgGpdSp~} \rightarrow$ sAlgSp to the category of simplicial algebraic spaces gives an isomorphism

$$
B^{1} \mathfrak{D} \mathfrak{e f} \mathfrak{f l g G p d S p}_{\operatorname{Alg}}^{2}(G) \cong \underline{\mathfrak{D} \mathfrak{e f}}_{s \operatorname{AlgSp}}(B G),
$$

between the 2-groupoid of deformations in AlgGpdSp, and the simplicial groupoid of deformations in sAlgSp.

Proof. By [Aok] Corollary 3.1.5, we know that $\Pi_{0} \mathfrak{D e f}_{\text {AlgGpdSp }}^{2}(G) \cong \Pi_{0} \underline{\mathfrak{D e f}}_{s \mathrm{AlgSp}}(B G)$, so we just need to show that, for algebraic groupoid spaces $H, G$,

$$
\underline{\operatorname{Hom}}_{s \operatorname{Algp}}(B H, B G)=B \mathscr{H} o m_{\mathrm{AlgGpdSp}}(H, G) .
$$

Now, $\underline{\operatorname{Hom}}_{s \operatorname{AlgSp}}(X, B G)_{n}=\operatorname{Hom}_{s \operatorname{AlgSp}}\left(X \times \Delta^{n}, B G\right)=\operatorname{Hom}_{\operatorname{AlgGpdSp}}\left(\pi_{f} X \times \pi_{f} \Delta^{n}, G\right)$, where we define the fundamental groupoid $\pi_{f}: s$ AlgSp $\rightarrow$ AlgGpdSp to be left adjoint to $B$, noting that $\pi_{f} B H=H$. However, $\pi_{f} \Delta^{n}$ is the groupoid with $n+1$ objects, and unique isomorphisms between them. Thus

$$
\operatorname{Hom}_{\mathrm{AlgGpdSp}}\left(\pi_{f} X \times \pi_{f} \Delta^{n}, G\right)=B_{n} \mathscr{H} o m_{\mathrm{AlgGpdSp}}\left(\pi_{f} X, G\right),
$$

as required.

Lemma 5.35. The functor $C$ defined in [Aok] §3.2 gives an equivalence between $\mathfrak{D} \mathfrak{e f} f_{\mathrm{AlgGpdSp}}^{2}(G)$ and $\mathfrak{D} \mathfrak{e f}^{2}(C G)$, the 2-groupoid of deformations of the algebraic stack $C G$. 
Proof. First observe that $C$ maps the 2-isomorphisms of Definition 5.32 to 2-isomorphisms of stacks, so $C$ is well-defined.

By [Aok] Proposition 3.2.5, we know that $C$ induces a bijection on isomorphism classes of objects. We need to show that for $\mathcal{G} \in \mathfrak{D e f}_{\text {AlgGpdSp }}^{2}(G)(A)$,

$$
C: \mathscr{H}_{o m m_{\mathfrak{A l g G p d S}}^{2}(G)(A)}(\mathcal{G}, \mathcal{G}) \rightarrow \mathscr{H}_{0} m_{\mathfrak{D e f}^{2}(C G)(A)}(C \mathcal{G}, C \mathcal{G})
$$

is an equivalence of groupoids. By [ibid.] Proposition 3.3.2, it is essentially surjective.

Given $f \in \mathrm{Ob} \mathscr{H}_{0 m^{D e f} f_{\text {AlgGpdS }}^{2}(G)(A)}(\mathcal{G}, \mathcal{G})$, we thus need to show that

$$
\theta: \operatorname{Aut}_{\mathfrak{D} \mathfrak{e f} \mathfrak{A l g G p d s p}^{2}(\mathcal{G})(A)}^{2}(f) \rightarrow \operatorname{Aut}_{\mathfrak{D} \mathfrak{e f}{ }^{2}(C \mathcal{G})(A)}^{2}(C f)
$$

is an isomorphism of 2-automorphism groups. Multiplication by $f^{-1}$ allows us to assume that $f=\operatorname{id}_{\mathcal{G}}$.

By [ibid.] Proposition 3.3.2, we have an exact sequence

$$
0 \rightarrow \operatorname{Aut}_{\mathfrak{D e f}^{2}(C \mathcal{G})}^{2}\left(\operatorname{id}_{C \mathcal{G}}\right) \rightarrow \operatorname{Aut}\left(X_{0} / C \mathcal{G}\right)_{P} \stackrel{A}{\rightarrow} \operatorname{Aut}_{\mathfrak{D e f}_{\mathrm{AlgGpdsp}}^{2}(G)}(\mathcal{G}) .
$$

Since $\operatorname{Aut}\left(X_{0} / C \mathcal{G}\right)_{P}$ is smooth, the homogeneous functor $\operatorname{Aut}_{\mathfrak{D e f}^{2}(C \mathcal{G})}^{2}\left(\operatorname{id}_{C \mathcal{G}}\right)$ has tangent space $\operatorname{ker}(\tan A)$ and obstruction space $\operatorname{coker}(\tan A)$. By [ibid.] Proposition 3.4.2, these are $\operatorname{Ext}^{-1}\left(\mathbb{L}_{\mathfrak{X}}, \mathscr{O}_{f X}\right)$ and $\operatorname{Ext}^{0}\left(\mathbb{L}_{\mathfrak{X}}, \mathscr{O}_{f X}\right)$, respectively. Thus $\S 5.2 .1$, Lemma 5.31 and Theorem 1.28 imply that $\theta$ gives isomorphisms on tangent and obstruction spaces, so must be an isomorphism of homogeneous functors by the standard smoothness criterion.

Taking $G=X_{0} \times_{\mathfrak{X}} X_{0} \Longrightarrow X_{0}$, we have therefore shown that the deformation 2groupoid of $\mathfrak{X}$ is equivalent to the simplicial deformation groupoid of $\operatorname{cosk}_{0}^{\mathfrak{X}}\left(X_{0}\right)$. We still need to compare this with the simplicial affine scheme $X_{\bullet}$ defined at the beginning of the section.

Proposition 5.36. The simplicial deformation groupoids of $\operatorname{cosk}_{0}^{\mathfrak{X}}\left(X_{0}\right)$ and $X_{\bullet}$ are equivalent.

Proof. Let $Z_{\bullet}:=\operatorname{cosk}_{0}^{\mathfrak{X}}\left(X_{0}\right)$. As in $\S 3.3$, we will consider three simplicial deformation problems $F_{X}, F_{Z}, F_{r}$ : deformations of $X_{\bullet}$, deformations of $Z_{\bullet}$, and deformations of the diagram $r: X_{\bullet} \rightarrow Z_{\bullet}$. Note that these all define quasi-smooth functors $F: \mathcal{C}_{\Lambda} \rightarrow$ $s \mathrm{Gpd} \stackrel{\bar{W}}{\longrightarrow} \mathbb{S}$, so we just need to compare tangent and obstruction spaces.

The calculations of Proposition 5.29 show that the loop spaces $\Omega F_{X}, \Omega F_{Z}$ have tangent spaces

$$
\underline{\operatorname{Hom}}_{\mathscr{O}_{X}}\left(\Omega_{X}, \mathscr{O}_{X}\right), \quad \underline{\operatorname{Hom}}_{\mathscr{O}_{Z}}\left(\Omega_{Z}, \mathscr{O}_{Z}\right) .
$$

Similarly, there is a fibration $F_{r} \rightarrow F_{X} \times F_{Z}$, whose fibre has tangent space

$$
\underline{\operatorname{Hom}}_{\mathscr{O}_{Z}}\left(\Omega_{Z}, r_{*} \mathscr{O}_{X}\right) \text {. }
$$

Since the maps

$$
\underline{\operatorname{Hom}}_{\mathscr{O}_{X}}\left(\Omega_{X}, \mathscr{O}_{X}\right) \stackrel{r_{*}}{\longrightarrow} \underline{\operatorname{Hom}}_{\mathscr{O}_{Z}}\left(\Omega_{Z}, r_{*} \mathscr{O}_{X}\right) \stackrel{r^{*}}{\longleftarrow} \underline{\operatorname{Hom}}_{\mathscr{O}_{Z}}\left(\Omega_{Z}, \mathscr{O}_{Z}\right)
$$

are isomorphisms, we deduce that the maps $F_{Z} \leftarrow F_{r} \rightarrow F_{X}$ induce isomorphisms on tangent spaces of positive homotopy groups.

It only remains to show that the deformation functors $\pi_{0} F_{X}, \pi_{0} F_{Z}, \pi_{0} F_{r}$ have isomorphic tangent and obstruction spaces. By adapting [Pri2] §1.3.1, we may deduce that these are (respectively)

$$
\operatorname{Ext}_{\mathscr{O}_{X}}^{i}\left(\mathbb{L}_{X}, \mathscr{O}_{X}\right), \quad \operatorname{Ext}_{\mathscr{O}_{Z}}^{i}\left(\mathbb{L}_{Z}, \mathscr{O}_{Z}\right)
$$

and the groups $T^{i}$ fitting into the long exact sequence

$$
\ldots \rightarrow T^{1} \rightarrow \operatorname{Ext}_{\mathscr{O}_{X}}^{1}\left(\mathbb{L}_{X}, \mathscr{O}_{X}\right) \times \operatorname{Ext}_{\mathscr{O}_{Z}}^{1}\left(\mathbb{L}_{Z}, \mathscr{O}_{Z}\right) \rightarrow \operatorname{Ext}_{\mathscr{O}_{Z}}^{1}\left(\mathbb{L}_{Z}, r_{*} \mathscr{O}_{X}\right) \rightarrow T^{2} \rightarrow \ldots
$$


Now, since the maps

$$
\operatorname{Ext}_{\mathscr{O}_{X}}^{i}\left(\mathbb{L}_{X}, \mathscr{O}_{X}\right) \stackrel{r_{*}}{\rightarrow} \operatorname{Ext}_{\mathscr{O}_{Z}}^{i}\left(\mathbb{L}_{Z}, r_{*} \mathscr{O}_{X}\right) \stackrel{r^{*}}{\longleftarrow} \operatorname{Ext}_{\mathscr{O}_{Z}}^{1}\left(\mathbb{L}_{Z}, \mathscr{O}_{Z}\right)
$$

are isomorphisms for $i \geq 1$, with $r_{*}$ surjective for $i=0$, we see that the functors $F_{r}, F_{X}, F_{Z}$ are all equivalent.

5.3. Arbitrary algebraic stacks. We now wish to describe derived deformations of a simplicial scheme $X_{\bullet}$ over $k$, with each $X_{n}$ a disjoint union of affine schemes.

Definition 5.37. For any scheme $Y$, let $\pi(Y)$ be the set of connected components of $Y$, and $\pi: Y \rightarrow \pi(Y)$ the map of associated topological spaces.

Now, deformations of $X_{n}$ are equivalent to deformations of the algebra $\pi_{*} \mathscr{O}_{X_{n}}$ over $\pi\left(X_{n}\right)$.

Definition 5.38. Recall that the ordinal number categories $\Delta, \Delta^{*}$ can be regarded as subcategories of $\mathbb{S}$, by identifying $[n]$ with $\Delta^{n}$.

Given a category $\mathcal{C}$ and $K \in \mathbb{S}$, define $c \mathcal{C}^{K}$ (resp. $c_{+} \mathcal{C}^{K}$ ) to be the category of functors from $\Delta \downarrow K$ (resp. $\Delta_{*} \downarrow K$ ) to $\mathcal{C}$. Thus an object $C \in c \mathcal{C}^{K}$ consists of objects $C_{a}$ for all $n \in \mathbb{N}_{0}, a \in K_{n}$, together with compatible maps $\partial^{i}: M_{\partial_{i} a} \rightarrow M_{a}, \sigma^{i}: M_{\sigma_{i} a} \rightarrow M_{a}$, and similarly for $c_{+} \mathcal{C}^{K}$.

Now, observe that $\pi_{*} \mathscr{O}_{X}$ defines an object of $c \operatorname{Alg}(k)^{\pi(X)}$, with

$$
\left(\pi_{*} \mathscr{O}_{X}\right)_{a}=\Gamma\left(\pi^{-1}(a), \mathscr{O}_{X_{n}}\right)
$$

for $a \in \pi\left(X_{n}\right)$. Since any deformation of $X_{\bullet}$ will not change $\pi(X)$, deformations of $X_{\bullet}$ are equivalent to deformations of $\pi_{*} \mathscr{O}_{X}$.

The categories $c \mathrm{FAlg}^{\pi(X)}, c_{+} \mathrm{FAlg}^{\pi(X)}, c \mathrm{FMod}^{\pi(X)}, c_{+} \mathrm{FMod}^{\pi(X)}$ can all be given simplicial structures as in Definition 5.1, setting $\left(C^{K}\right)_{a}=\left(C_{a}\right)^{K_{n}}$ for $a \in \pi\left(X_{n}\right)$.

Remark 5.39. Observe that for any category $\mathcal{C}$ and any map $f: K \rightarrow L$ in $\mathbb{S}$, there are maps $f^{-1}: c \mathcal{C}^{L} \rightarrow c \mathcal{C}^{K}, f^{-1}: c_{+} \mathcal{C}^{L} \rightarrow c_{+} \mathcal{C}^{K}$ given by $\left(f^{-1} C\right)_{a}=C_{f(a)}$. If $\mathcal{C}$ contains products, then $f^{-1}$ has a right adjoint $f_{*}$, given by $\left(f_{*} C\right)_{b}=\prod_{a \in f^{-1}(b)} C_{a}$. For $f: K \times L \rightarrow L$, $C^{K}=f_{*} f^{-1} C$.

If $f: \pi(X) \rightarrow \bullet$ denotes the constant map, then we write $\Gamma:=f_{*}$, with the constant functor $f^{-1}$ denoted by $\Gamma^{*}$.

We then have a diagram of adjunctions of functors $\mathcal{C}_{\Lambda} \rightarrow s$ Cat:

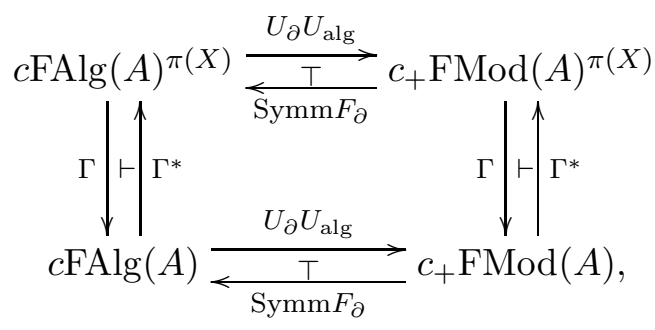

where $F_{\partial}: c_{+} \mathcal{C}^{\pi(X)} \rightarrow c \mathcal{C}^{\pi(X)}$ is left adjoint to the forgetful functor $U_{\partial}$, given by

$$
\left(F_{\partial} C^{*}\right)_{a}=C_{a} \sqcup C_{\partial_{0} a} \sqcup \ldots \sqcup C_{\left(\partial_{0}\right)^{n} a},
$$

for $a \in \pi\left(X_{n}\right)$, with operations dual to those in Lemma 4.12.

We must check that $\Gamma^{*} \dashv \Gamma$ is monadic. For this, we verify Beck's Theorem (e.g. [Mac] Ch. VI.7 Ex. 6), observing that $\Gamma$ commutes with coequalisers - this is effectively the observation that taking arbitrary products is an exact functor.

Writing $U:=U_{\partial} U_{\text {alg }}$ and $F:=\operatorname{Symm} F_{\partial}$, we also have the following commutativity conditions:

$$
\Gamma U=U \Gamma \quad \Gamma^{*} F=F \Gamma^{*}, \quad \Gamma^{*} U=U \Gamma^{*},
$$


and a natural transformation

$$
F \Gamma \rightarrow \Gamma F .
$$

These adjunctions combine to give a monadic adjunction

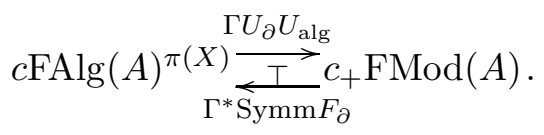

Definition 5.40. Given a simplicial scheme $X_{\bullet}$, with each $X_{n}$ a disjoint union of affine schemes, define $c \operatorname{Mod}(X)$ to be the category of $\pi_{*}\left(\mathscr{O}_{X}\right)$-modules over $c \operatorname{Mod}^{\pi(X)}$.

Lemma 5.41. If $X \rightarrow Y$ is a trivially smooth map of simplicial schemes, with each $X_{n}, Y_{n}$ a disjoint union of affine schemes, then $\pi_{*} \Omega(X / Y)$ is projective in cMod $(X)$.

Proof. This is similar to Lemma 5.14. We may define matching objects of $L \in c \operatorname{Mod}(X)$ by letting $M^{n} L$ on $\pi\left(X_{n+1}\right)$ be the equaliser

$$
M^{n} L \longrightarrow \prod_{i=0}^{n} \sigma_{i *} L^{n} \stackrel{a}{\underset{b}{\longrightarrow}} \prod_{0 \leq i<j \leq n} \sigma_{i *} \sigma_{j *} L^{n-1},
$$

where $\operatorname{pr}_{i j} \circ a=\sigma^{i} \circ \mathrm{pr}_{j}, \operatorname{pr}_{i j} \circ b=\sigma^{j-1} \circ \mathrm{pr}_{i}$. Note that $\Gamma\left(M^{n} L\right)=M^{n}(\Gamma L)$. Since $\Gamma$ reflects isomorphisms, this means that for all surjections $L \rightarrow N$, the relative matching map $L^{n} \rightarrow M^{n-1} L \times{ }_{M^{n-1} N} N^{n}$ is surjective.

In order to construct latching maps, note that any cocontinuous functor $S:(\Delta \downarrow$ $\pi(X)) \rightarrow$ Alg extends to a cocontinuous functor $S:(\mathbb{S} \downarrow \pi(X)) \rightarrow$ Alg. Given $M^{\bullet} \in$ $c \operatorname{Mod}(X)$ and $a: K \rightarrow \pi(X)$ in $\mathbb{S}$, define $M(a) \in \operatorname{Mod}\left(\pi_{*}\left(\mathscr{O}_{X}\right)(a)\right)$ by

$$
\pi_{*}\left(\mathscr{O}_{X}\right)(a) \oplus M(a) \epsilon=\left(\pi_{*}\left(\mathscr{O}_{X}\right) \oplus M \epsilon\right)(a) .
$$

Note that if we set $X(a):=\operatorname{Hom}_{\mathbb{S} \mid \pi(X)}(K, X)$, then $\Omega(X / Y)(a)=\Omega\left(X(a) / Y\left(\pi(f)_{*} a\right)\right)$.

The latching object of $\Omega(a)$, for $a \in \pi(X)_{n}$, is $\Omega(\partial a)$, for $\partial: \partial \Delta^{n} \rightarrow \Delta^{n}$. It therefore suffices to show that $\left(X^{\partial}\right)^{*} \Omega(\partial a) \rightarrow \Omega(a)$ is projective in $\operatorname{Mod}(X(a))$ for all such $a$. By adapting the proof of Lemma 5.14, it suffices to show that

$$
X(a) \rightarrow Y\left(\pi(f)_{*} a\right) \times_{Y(\pi(f) * \partial a)} X(\partial a)
$$

is smooth.

Set $Y^{\prime}:=X \times_{\pi(X)} \pi(Y)$, and observe that Lemma 5.12 implies that $X \rightarrow Y^{\prime}$ is trivially smooth. Thus the matching map $X_{n} \rightarrow M_{n} X \times_{M_{n} Y^{\prime}} Y_{n}^{\prime}$ is smooth. The required result is then obtained by taking the fibre over $a \in \pi(X)_{n}$.

Lemma 5.42. If we set $\perp^{\prime}=\Gamma^{*} F U \Gamma$, and

$$
\mathbb{L}_{\bullet}^{\perp^{\prime}}(X):=\Omega\left(\left(\perp^{\prime}\right)^{n+1} \pi_{*}\left(\mathscr{O}_{X}\right)\right) \otimes_{\left(\perp^{\prime}\right)^{n+1} \pi_{*}\left(\mathscr{O}_{X}\right)} \pi_{*}\left(\mathscr{O}_{X}\right),
$$

then for all $m$, the simplicial complex $\mathbb{L}_{\bullet}^{\perp^{\prime}}(X)^{m}$ is a model for the cotangent complex of $X_{m}$.

Proof. This is essentially the same as Lemma 5.7, making use of the observation that $\Gamma$ is exact and reflects isomorphisms, so it suffices to prove that $U \Gamma\left(\perp^{\prime}\right)^{\bullet+1} \pi_{*}\left(\mathscr{O}_{X}\right) \rightarrow$ $U \Gamma \pi_{*}\left(\mathscr{O}_{X}\right)$ is a resolution.

Definition 5.43. Define $\mathcal{D}$ to be the simplicial category of pairs $(K, R)$, for $K \in \mathbb{S}$, $R \in\left(c \mathrm{FAlg}^{K}\right)^{\mathrm{opp}}$, with a morphism $f \in \underline{\operatorname{Hom}}_{\mathcal{D}}((K, R),(L, S))_{n}$ consisting of $f: K \rightarrow L$ in $\mathbb{S}$, together with $f^{\sharp} \in \underline{\operatorname{Hom}}_{c \mathrm{FAlg}}{ }^{K}\left(f^{-1} S, R\right)_{n}$.

Define $\mathcal{B}:=\mathbb{S} \times\left(c_{+} \mathrm{Mod}\right)^{\mathrm{opp}}$, with simplicial structure coming from $\left(c_{+} \mathrm{Mod}\right)^{\mathrm{opp}}$.

Now, observe that we have a forgetful functor $V: \mathcal{D} \rightarrow \mathcal{B}$, given by $(K, R) \mapsto$ $\left(K, \Gamma U_{\partial} U_{\text {alg }} R\right)$, with right adjoint $G: \mathcal{B} \rightarrow \mathcal{D}$ given by $(K, M) \mapsto\left(K, \Gamma^{*} \operatorname{Symm} F_{\partial} M\right)$. We have already seen that this adjunction is comonadic (by fixing $K$ ). 
Proposition 5.44. If $X, Y$ are simplicial schemes over $k$, with each $X_{n}, Y_{n}$ a disjoint union of affine schemes, and $X$ quasi-smooth, then every morphism $\rho: Y \rightarrow X$ is quasismooth over $\mathcal{B}$, in the sense of Definition 3.22.

Proof. The proof of Proposition 5.19 carries over, using Lemmas 5.41 and 5.42 instead of Lemmas 5.14 and 5.7 .

Corollary 5.45. For any diagram in $\mathcal{D}(k)$ with quasi-smooth objects, the associated pre$D D C$ given by Definition 3.24 and Proposition 3.19 applied to the adjunction $G \vdash V$ is a DDC by Lemma 3.25, and governs deformations in the simplicial category $\mathcal{D}$ (by Proposition 3.26).

\section{REFERENCES}

[Aok] Masao Aoki. Deformation theory of algebraic stacks. Compos. Math., 141(1):19-34, 2005.

[FM] D. Fiorenza and M. Manetti. L_o structures on mapping cones. Algebra Number Theory, 1(3):301330, 2007. arXiv math.QA/0601312.

[GJ] Paul G. Goerss and John F. Jardine. Simplicial homotopy theory, volume 174 of Progress in Mathematics. Birkhäuser Verlag, Basel, 1999.

[Gle] Paul G. Glenn. Realization of cohomology classes in arbitrary exact categories. J. Pure Appl. Algebra, 25(1):33-105, 1982.

[Gro] Alexander Grothendieck. Technique de descente et théorèmes d'existence en géométrie algébrique. II. Le théorème d'existence en théorie formelle des modules. In Séminaire Bourbaki, Vol. 5, pages Exp. No. 195, 369-390. Soc. Math. France, Paris, 1995.

[Hir] Philip S. Hirschhorn. Model categories and their localizations, volume 99 of Mathematical Surveys and Monographs. American Mathematical Society, Providence, RI, 2003.

[Ill] Luc Illusie. Complexe cotangent et déformations. I. Springer-Verlag, Berlin, 1971. Lecture Notes in Mathematics, Vol. 239.

[Mac] Saunders MacLane. Categories for the working mathematician. Springer-Verlag, New York, 1971. Graduate Texts in Mathematics, Vol. 5.

[Man] Marco Manetti. Deformation theory via differential graded Lie algebras. In Algebraic Geometry Seminars, 1998-1999 (Italian) (Pisa), pages 21-48. Scuola Norm. Sup., Pisa, 1999. arXiv math.AG/0507284.

[Ols1] Martin Olsson. Sheaves on Artin stacks. J. Reine Angew. Math., 603:55-112, 2007.

[Ols2] Martin C. Olsson. Deformation theory of representable morphisms of algebraic stacks. Math. Z., 253(1):25-62, 2006.

[Pri1] J. P. Pridham. Deforming $l$-adic representations of the fundamental group of a smooth variety. $J$. Algebraic Geom., 15(3):415-442, 2006.

[Pri2] J. P. Pridham. Deformations of schemes and other bialgebraic structures. Trans. Amer. Math. Soc., 360(3):1601-1629, 2008.

[Pri3] J. P. Pridham. The homotopy theory of strong homotopy algebras and bialgebras. Homology, Homotopy Appl., 12(2):39-108, 2010. arXiv:0908.0116v2 [math.AG].

[Pri4] J. P. Pridham. Unifying derived deformation theories. Adv. Math., 224(3):772-826, 2010. arXiv:0705.0344v5 [math.AG].

[Pri5] J. P. Pridham. Derived deformations of schemes. Comm. Anal. Geom., 20(3):529-563, 2012. arXiv:0908.1963v1 [math.AG].

[Pri6] J. P. Pridham. Presenting higher stacks as simplicial schemes. Adv. Math., to appear. arXiv:0905.4044v3 [math.AG].

[Qui] Daniel Quillen. Rational homotopy theory. Ann. of Math. (2), 90:205-295, 1969.

[Ran] Ziv Ran. Lie atoms and their deformations. Geom. Funct. Anal., 18(1):184-221, 2008. arXiv:math/0412204v7.

[Van] Donovan H. Van Osdol. Bicohomology theory. Trans. Amer. Math. Soc., 183:449-476, 1973.

[Wei] Charles A. Weibel. An introduction to homological algebra. Cambridge University Press, Cambridge, 1994. 